\title{
Comparative Transcriptome Analysis Reveals Molecular Mechanism and Regulatory Network of Rosa Chinensis 'Old Blush' Leaves in Response to Drought Stress
}

\section{Xin Jia}

Beijing Forestry University

Hui Feng

Beijing Institute of Landscape Architecture

Yanhua Bu

Beijing Institute of Landscape Architecture

Yingmin Lu

Beijing Forestry University https://orcid.org/0000-0002-9087-8508

Naizhe Ji

Beijing Institute of Landscape Architecture

Shiwei Zhao ( $\nabla$ zhaoshiwei@beijingbg.com )

Beijing Institute of Landscape Architecture https://orcid.org/0000-0001-5636-0855

\section{Research article}

Keywords: drought stress, comparative transcriptome analysis, WGCNA, Rose, transcription factors

Posted Date: November 24th, 2020

DOI: https://doi.org/10.21203/rs.3.rs-112819/v1

License: (c) (i) This work is licensed under a Creative Commons Attribution 4.0 International License.

Read Full License 


\section{Abstract}

Background: Rose is one of the most important ornamental crops, but drought limits its yield and quality seriously. Although serval abiotic stress related genes have been identified, the molecular mechanism during rose's responding to drought stress is still limited. In this study, the transcriptomes of leaves of two-year-old cutting seedlings of Rosa chinensis 'Old Blush' from three continuous droughted stages (30, 60,90 days after full watering) and rewatering were analyzed using RNA sequencing. Meanwhile, weighted gene co-expression network analysis (WGCNA) was used to construct a co-expression network associated with the physiological traits of drought response to discover the hub transcription factors involved in drought response.

Results: More than 45 million high-quality clean reads were generated from samples and used for comparison with the rose reference genome. A total of 46433 differentially expressed genes were identified. Gene ontology (GO) term enrichment and Kyoto Encyclopedia of Genes and Genomes (KEGG) analysis indicated that drought stress caused significant changes in signal transduction, plant hormones, primary and secondary metabolism, and a certain degree of recovery after rewatering. Gene co-expression analysis identified 18 modules, in which four modules have highly degree of correlation with physiological traits. In addition, twenty-two transcription factors including members of NACs, WRKYS, $M Y B s, E R F s, A R F s$ and $b H L H s$ with high connectivity in these four modules were screened.

Conclusions: The results of this study provided the first transcriptome sequencing report of the drought stress in R.chinensis, and also provided candidate transcription factors in response to drought stress, providing clues for improving the drought tolerance of the rose through molecular breeding in the future.

\section{Background}

Rose is the world's most important ornamental plant, with economic, cultural, and symbolic value, and occupies the major position in the floriculture industry. However, their growth conditions are closely related to the changes of external environment. Most of roses are generally suffered from varying degrees of drought inducing the loss of productivities and quality around the world. As a kind of abiotic stresses, drought is becoming a common environmental stress with the global warming changing Earth's climate which adversely impacts on plant growth, crop production, and distribution [1]. Thence, it is pressing to identify the response mechanism in rose under the drought stress, with the limiting understand so far.

To resistance the drought stress, plants have evolved a number of adaptive mechanisms depending upon the activation of molecular networks involved in stress perception, signal transcription and transduction, expression of specific stress-related genes and physiological reaction of tolerance [2]. It is significant that identified the genes and biological pathways involving in drought stress tolerance, improving our understanding of the molecular mechanisms relating to drought resistance in plants [3]. Gazillions of signaling and metabolic pathways and numerous genes have been determined in several model plants exposed in drought environment, such as Arabidopsis [4], rice [5], and so on. However, these genes are not 
enough to explain the complete drought response mechanism. Different species may have different drought response pathways.

As a deep sequencing technology, transcriptome technology has been successfully used to explore the gene network of non-model plant species in response to drought stress. Recently, drought-responsive transcriptome studies have been conducted in ornamental plants such as chrysanthemum [6], tree peony (Paeonia section Moutan DC.) [7]and Dianthus spiculifolius [8]. Previously, only a suppression subtractive hybridization library was constructed for the dehydration stress of cut rose (Rosa hybrida cv. Samantha) petals, which contained 3513 unique expression sequence tags, and its expression profile during the dehydration cycle was analyzed [9]. Currently, the genome of R.chinensis 'Old Blush' was published, providing a valuable database for the further function and genomic research in rose [10]. To date, $R C L E A$ [11], RcMYBPA2 [12], RhMYB96 [13], RcNAC3 [14] and RcNAC31 [15] genes have been reported to improve the abiotic stress resistance of transgenic plant. However, there is still limited information on the mechanism of rose response to drought stress.

R.chinensis 'Old Blush', originating from China, participating in modern rose hybrid breeding, is the diploid species in Rosa, R.chinensis 'Old Blush' may represent a convenient genomic model for Rosa research. It may be used as a model plant to study rose response to drought stress. In order to obtain unique insights into the molecular mechanisms at the transcriptome level in response to drought, we compared transcriptome profiles of R.chinensis 'Old Blush' at different drought stages (30,60,90 days after full watering) and rewatering. We used this information to summary the major drought responsive processes in R.chinensis and identify hub genes through WGCNA. The goal of this study was to obtain understanding of the drought responsive regulatory network at the molecular level. Besides, the results of this study provide a basis for a better understanding of not only other R.hybrida cultivars but also other woody plants responding to drought stress, because rose is also considered as a good object for studying woody ornamental plants [16].

\section{Results}

\section{Changes in phenotype and physiology at drought stress in R.chinensis}

We applied drought stress by stopping watering on R.chinensis. The plant leaf phenotypes were visually inspected and physiologically measured under control condition (CK), and at 15,30,45,60,75,90 days after stress and rewatering was applied. Under normal circumstances, the leaves were dark green and shiny (Fig. 1a). After 15 days of drought treatment, the leaves had no obvious morphological changes (Fig. 1b). The leaf relative water content is a quantitative indicator of plant water status [17]. Similarly, the relative soil water content and leaf relative water content were not significantly different from the control (Fig. 2a, Fig. 2b). At 30 days the leaves still appeared healthy, but the leaves were slightly wrinkled (Fig. 1c). When the drought stress lasted for 45 days and 60 days, the leaves were all curled (Fig. 1d, Fig. 1e). At this time, the relative water content of the soil decreased significantly, reaching a moderate degree of stress 
(Fig. 2a). At 60 days, the relative water content of the leaves decreased significantly compared with the control, which was consistent with the phenotype (Fig. 2b). The curl degree of the leaf deepened and the leaf became thinner, and the relative water content of the leaf was significantly reduced during 75 days of drought (Fig. 1f, Fig. 2b). However, after 90 days of drought, both the leaf relative water content and the soil relative water content reached the lowest, reaching the level of severe stress. At the same time, the leaves were severely curled and withered (Fig. 1g, Fig. 2a, Fig. 2b). After rewatering, the leaves were green and thin (Fig. 1h). In order to determine the exact point for transcriptome sequencing, we also measured physiological indicators. Malondialdehyde (MDA) is usually an important physiological indicator to measure oxidative damage under abiotic and biological stress. The MDA content of leaves treated with drought and rewatering was determined. With the deepening of the degree of drought stress, the content of MDA rises in fluctuation and got the highest after 90 days of the drought treatment (Fig. 2d). In addition, the content of superoxide dismutase (SOD) was examined and it was found that SOD increased with the increasing of drought treatment time and reached the maximum value after 90 days (Fig. 2c). We also found that both MDA and SOD content increased significantly during drought treatment for 30 days, 60 days and 90 days. After rewatering, the MDA and SOD contents were all reduced. Based on these results, we selected leaves under normal conditions, drought treatment for 30 days, 60 days, 90 days, and rewatering treatment for transcriptome sequencing.

\section{Transcriptome analysis of R.chinensis}

To investigate genes involved in the transcriptome, a cDNA sample was prepared from an equal mixture of total RNA isolated from the leaves of 2-year-old cutting seedlings R.chinensis from the control, drought-treated (30,60,90 d) and rehydration groups, designated as CK1, CK2, CK3, DT1-1, DT1-2, DT1-3, DT2-1, DT2-2, DT2-3, DT3-1, DT3-2, DT3-3, RW1, RW2, RW3. Three biological replicates from each group were analyzed on the Illumina HiSeq 4000 platform. The clean reads removing adaptor sequences, ambiguous nucleotides, and low-quality reads ( $Q$ value < 20) ranged from 41397356 to 54756474 . The Q20 and Q30 values of all 15 libraries were more than $97 \%$ and $92 \%$. The GC content was approximately ranged from $48-49 \%$. Between $92.33 \%$ and $93.89 \%$ of the sequenced reads could be aligned to the rose reference genome (Table 1). In this study, the proportion of exon sequences ranged from 95.30-96.38\%, and the proportion of intron sequences ranged from 1.45-2.66\% (Additional file 1: figure S4). 
Table 1

RNA sequencing data and corresponding quality control

\begin{tabular}{|lllllll|}
\hline $\begin{array}{l}\text { Sample } \\
\text { name }\end{array}$ & $\begin{array}{l}\text { Clean } \\
\text { reads }\end{array}$ & $\begin{array}{l}\text { Raw } \\
\text { reads }\end{array}$ & Mapped reads & $\begin{array}{l}\text { GC Content } \\
(\%)\end{array}$ & Q20(\%) & Q30(\%) \\
\hline CK1 & 48126136 & 48181768 & $\begin{array}{l}44510062 \\
(93.27 \%)\end{array}$ & 48.28 & 97.51 & 92.92 \\
\hline CK2 & 49106032 & 49156710 & $\begin{array}{l}45429755 \\
(93.89 \%)\end{array}$ & 48.66 & 98.03 & 94.17 \\
\hline CK3 & 43793126 & 43853234 & $\begin{array}{l}40291282 \\
(93.01 \%)\end{array}$ & 48.29 & 97.48 & 92.88 \\
\hline DT1-1 & 45651270 & 45706946 & $\begin{array}{l}42334367 \\
(93.31 \%)\end{array}$ & 49.52 & 97.67 & 93.28 \\
\hline DT1-2 & 48888834 & 48972378 & $\begin{array}{l}44986749 \\
(92.70 \%)\end{array}$ & 48.11 & 97.38 & 92.70 \\
\hline DT1-3 & 53820314 & 53902378 & $\begin{array}{l}49546849 \\
(92.94 \%)\end{array}$ & 48.30 & 97.46 & 92.85 \\
\hline DT2-1 & 47463436 & 47521292 & $\begin{array}{l}43862640 \\
(93.07 \%)\end{array}$ & 48.19 & 98.12 & 94.31 \\
\hline DT2-2 & 54353944 & 54420772 & $\begin{array}{l}49969726 \\
(93.02 \%)\end{array}$ & 48.23 & 98.10 & 94.27 \\
\hline DT2-3 & 51937470 & 52002482 & $\begin{array}{l}47795161 \\
(92.33 \%)\end{array}$ & 48.09 & 98.13 & 94.32 \\
\hline DT3-1 & 50304612 & 50359618 & $\begin{array}{l}40931525 \\
(93.07 \%)\end{array}$ & 49.61 & 98.27 & 94.71 \\
\hline DT3-2 & 44365148 & 44421624 & $\begin{array}{l}38029228 \\
(93.35 \%)\end{array}$ & 49.58 & 98.10 & 94.28 \\
\hline DT3-3 & 49072308 & 49130228 & $\begin{array}{l}41525793 \\
(93.40 \%)\end{array}$ & 49.60 & 98.18 & 94.50 \\
\hline RW1 & 54756474 & 54838556 & $\begin{array}{l}50746877 \\
(93.11 \%)\end{array}$ & 48.65 & 94.00 \\
\hline RW2 & 41397356 & 41446080 & $\begin{array}{l}38442300 \\
(93.52 \%)\end{array}$ & & & \\
\hline
\end{tabular}

\section{Comparisons of differentially expressed genes under the different drought stress stages}


To analyze the differentially expressed genes (DEGs) between leaves suffered drought stress at four different time duration (30,60,90 $\mathrm{d}$ and rewatering) and the control, we conducted comparisons between five groups. The DEGs were filtrated according to an expression level $|\log 2(F C)|>1$ and FDR $<0.05$ in each pairwise comparison. 6380 genes were identified exclusively at DT3, while 866 genes were specific in DT1. Especially 6380 (35.14\%) genes were identified in DT3, but only 1680 (9.25\%) and 1804 (9.94\%) genes were identified in DT1 and DT2 (Fig. 3a).

The number of DEGs is an increase from DT1 to DT3, reached a peak at CK vs. DT3 comparison set, indicated that most of the drought regulated genes are late-response. The results showed DEG enrichment in CK vs. DT1, CK vs. DT2 and CK vs. DT3, illustrating that the number of down-regulated DEGs was significantly higher than the number of upregulated DGEs. Meanwhile, a total of 11545 DEGs including 7886 downregulated and 3659 upregulated between CK and DT3 is the most among the drought stress groups. Meanwhile, only 2503 upregulated and 2367 downregulated between CK and DT1, which suggested that the differentiation of expressed genes between CK and DT3 is larger than that of CK and DT1, this revealed that, transcript abundance changed dramatically at key switched among the drought stages, which drought response genes could be induced and expressed largely. Comparing with CK, slightly more genes upregulated than genes downregulated in RW (Fig. 3b).

\section{GO functional enrichment and KEGG pathway enrichment analysis of DEGs}

According to GO functional enrichment analysis, as the drought stress deepened, the most enriched GO category among these DEGs was 'metabolic process', followed by 'cellular process', 'biological regulation', 'regulation of biological process', 'response to stimulus', 'catalytic activity', 'binding', 'transport activity', 'cell part', 'cell', 'organelle', 'membrane' and 'membrane part', so we can see, the drought resistance process in R.chinensis is complex. Meanwhile, according to the $\mathrm{GO}$ analysis of up-regulated DEGs, the $\mathrm{GO}$ terms of up-regulated DEGs in CK vs DT1,CK vs DT2 and CK vs RW were mostly concentrated on membranes, including the cellular components category, such as 'membrane'(GO:0016020), 'membrane part'(GO:0044425) and 'intrinsic component of membrane'(GO:0031224) (Fig. 4a, Fig. 4b, Fig. 5b). And CK vs DT3 involved more cells and metabolic processes including the biological process category (metabolic process, GO:0008152, cellular, G0:0009987, single-organism process, G0:0044699, cellular metabolic process, GO:0044237.) (Fig. 5a, Additional file 2: Table S1). In addition, the GO analysis of the down-regulated DEGs found that among the top 20 enriched, 13 belong to the category of biological processes, mainly focusing on the response to stimuli in CK vs DT1(Fig. 4a, Additional file 3: Table S2).

The KEGG pathway analysis revealed that it is mainly enriched in metabolism under the different drought degree and rewatering treatment. Among the top 10 enriched pathways, 'plant-pathogen interaction'(ko04626), 'biosynthesis of secondary metabolites'(ko01110), 'plant hormone signal transduction'(ko04075), 'MAPK signaling pathway-plant'(ko04016), were regulated in response to DT1, which suggested that drought stress had been sensed and signaling transduction pathways had been 
activated (Fig. 6a). However, more pathways related to metabolism were enriched at DT2 and DT3. The 'photosynthesis'(ko00195) and 'photosynthesis-antenna proteins'(ko00196) associated with photosynthesis were all enriched in CK vs DT2 and CK vs DT3 (Fig. 6b, Fig. 7a). Metabolite-related pathways such as 'biosynthesis of secondary metabolites'(ko01110), 'alpha-Linolenic acid metabolism'(ko00592), 'tryptophan metabolism'(ko00380), 'glycosphingolipid biosynthesis-lacto and neolacto series'(ko00601), 'alanine, aspartate and glutamate metabolism'(ko00250), 'galactose metabolism'(ko00052) and 'glyoxylate and dicarboxylate metabolism'(ko00630) were enriched in CK vs DT2 and CK vs DT3. These results indicated that metabolism processes may act as the predominant process of middle and late stages of drought stress in R.chinensis. It was unsurprising because drought causes changes in the metabolism of plants to improve their drought tolerance. Apart from pathways enriched metabolism, 'plant-pathogen interaction'(ko04626) was also enriched in CK vs RW, indicating that plants are vulnerable to pathogenic bacteria after recovering from drought stress (Fig. $7 \mathrm{~b}$, Additional file 4: Table S3).

\section{Identification of plant hormone and signal transduction- related DEGs}

Signal transduction in plants was evidently influenced by drought stress, as verified by both the GO and KEGG enrichment analyses of the DEGs performed in this study. More than 240 genes were predicted to encode protein kinases with varying expression levels in R.chinensis. Genes encoding receptor-like protein kinases $(R L K S)$ in which LRR receptor kinases were the majority accounted for the largest proportion of these genes. Twenty-nine of them were up-regulated in DT1 and DT2. Among them, MIK1(RchiOBHm_Chr6g0286591) was up-regulated 8.02-fold, respectively. More than 66 RLK genes were included in the group of serine/threonine-protein kinases during the drought stress. Furthermore, one gene related to G-type lectin S-receptor-like serine/threonine-protein kinase was found to show the highest degree of upregulation in DT3. Mitogen-activated protein kinase (MAPK) occupy an important role in signal transduction induced by abiotic stress [18]. The KEGG pathways analysis of the DEGs, the 'MAPK signaling pathway'(ko04016) was induced in the drought treatment. For DEGs detected in DT1 and DT2, two MAPKKKs including RchiOBHm_Chr2g0158821 and RchiOBHm_Chr5g0002581 and four MAPKKS including, MPK9(RchiOBHm_Chr7g0178621), MKK1O(RchiOBHm_Chr7g0211301), MEKK1(RchiOBHm_Chr7g0240941), MPK4(RchiOBHm_Chr7g0196781) were up-regulated. MKK1O(RchiOBHm_Chr7g0211301) was particularly up-regulated 9.18-fold. However, three MAPKKs consist of MPK3(RchiOBHm_Chr5g0061451), MKK4(RchiOBHm_Chr2g0150341) and MEKK1(RchiOBHm_Chr1g0352161) were down-regulated. In addition, MPK4(RchiOBHm_Chr7g0196781) and MPK19(RchiOBHm_Chr5g0028971) were substantially increased in DT3(Additional file 5: Table S4).

As a second messenger, calcium ion can not only maintain the stability of cell structure, but also play a vital role in signal transmission and participate in plant stress response $[19,20] . \mathrm{Ca}^{2+}$ signal transmission is achieved through the generation, decryption and transmission of specific calcium signals and the corresponding physiological and biochemical reactions downstream. In this study, we identified DEGs 
related to calcium ion, encoding calcium-binding proteins, $\mathrm{Ca}^{2+}$-binding protein $\mathrm{EF}$ hand, calmodulin-like, calcium-dependent protein kinases, calcium-transporting ATPase, and calcium channel protein. One gene (RchiOBHm_Chr2g0158571) encoding the calcium channel protein sustained high expression under drought stress and was up-regulated 6.44-fold in DT1 vs CK (Additional file 6: Table S5).

When plants were subjected to environmental stress, reactive oxygen species generated signals in the plant, triggering a series of changes. Plants also have an antioxidant system protecting against poisonous oxygen [21]. According to GO and KEGG analysis of the DEGs, we found that the GO term 'hydrogen peroxide metabolic'(G0:0042743) and 'response to reactive oxygen species'(GO:0000302) were enriched at DT1 and DT2, Peroxisome(ko04146) was induced at DT3. We identified 69 DEGs encoding ROS production and scavenging. A total of 55 DEGs encoding enzymes related to ROS scavenging, including peroxidase (POD), ascorbate peroxidase (APX), glutathione S-transferase (GST), glutathione peroxidase (GPX), polyphenol oxidase (PPO), ferritin, glutaredoxin, thioredoxin and peroxiredoxin. Among these antioxidant enzymes, POD45(RchiOBHm_Chr4g0400821) encoding peroxidase was up-regulated by 4.79-fold at DT1, another gene encoding peroxidase PNC1(RchiOBHm_Chr5g0072281) was no significant change from DT1 to the control, but it increased significantly 9.29-fold at DT2, reaching the peak of expression level at DT3 (Additional file 7: Table S6).

Moreover, the expression of genes involved in hormone biosynthesis or signaling was substantially changed following the drought stress and then rewatering, including $A B A$, auxin, brassinosteroid (BR), cytokinin, ethylene (ET), jasmonic acid (JA) and salicylic acid (SA). Among these DEGs, most genes were involved in ABA response. Nine-cis-epoxycarotenoid dioxygenase (NCED) is an important enzyme in ABA biosynthesis. For instance, one gene encoding NCED3 (RchiOBHm_Chr5g0014331) was up-regulated by 2.70-fold at DT1. Another gene encoding NCED6(RchiOBHm_Chr4g0397001) was significantly downregulated 9.40-fold at DT3. Interestingly, the expression of a gene (RchiOBHm_Chr5g0049951) encoding $A A O$, which is also a vital enzyme in ABA biosynthesis, was up-regulated by 2.78 -fold at DT1 and downregulated by 7.28 -fold at DT3. Moreover, some genes involved in ABA mediated signaling pathway were also induced by drought stress. Abscisic acid receptor PYL4 (RchiOBHm_Chr1g0371101) was downregulated. In contrast, one gene encoding PP2C (RchiOBHm_Chr5g0066401), a negative regulator of ABA, was up-regulated (Additional file 8: Table S7).

In total of 24 genes involved in the auxin signaling pathway were also changed. Three genes encoding auxin response factor ( $A R F 3, A R F 5, A R F 9$ ) were drought induced. Particularly, ARF3

(RchiOBHm_Chr5g0009381) expression continued to decline under drought stress, which was induced for 4.41-fold at DT3, while the expression level of ARF5 (RchiOBHm_Chr6g0302551) continued to increase under drought stress. Two genes encoding $A U X / I A A$ proteins were up-regulated at DT1 and downregulated at DT3. AUX22D (RchiOBHm_Chr4g0389611) was downed by nearly 10 -fold at DT3. In addition, some genes participated in auxin response and signal, such as auxin-binding protein ABP19a-like (ABP19A), auxin transporter-like protein 2 (LAX2) and auxin efflux carrier (PIN1C). The gene encoding $A B P 19 A$ (RchiOBHm_Chr2g0094781) was up-regulated 7.41-fold at DT1. Five genes encoding SAUR were 
identified, among these, SAUR77(RchiOBHm_Chr5g0026611) was up-regulated 9.78-fold at DT1 (Additional file 8: Table S7).

In this study, large numbers of ethylene-responsive transcription factor (ERF) genes displayed a trend of down-regulation under early drought stress. However, one gene encoding ERF023

(RchiOBHm_Chr1g0360021) was up-regulated 3.70-fold at DT1 and then down-regulated 5.02-fold at DT3. Another gene encoding ERF110 (RchiOBHm_Chr6g0274591) was up-regulated 7.34-fold at DT3. Meanwhile, we identified ethylene receptor (ERS1), ethylene-overproduction protein 1 (ETO1) and ethylene insensitive3-like protein (EIL3) (Additional file 8: Table S7).

Most of genes associated with the biosynthesis or signaling of jasmonic acid (JA) were down-regulated. The gene encoding malate dehydrogenase $(M D H G)$ was down-regulated 4.06-fold at DT3, while 12oxophytodienoate reductase 3-like (OPR3) was up-regulated 4.13-fold at DT3. Furthermore, the majorities of genes related to cytokinin, salicylic acid and brassinosteroid pathways. One gene encoding cytokinin dehydrogenase 3 (RchiOBHm_Chr2g0093491) was down-regulated 7.40-fold at DT3 (Additional file 8: Table S7).

\section{Expression of genes involved in metabolism and biosynthesis}

Through the GO and KEGG enrichment analysis, we found that many DEGs were related to metabolism and biosynthesis. The 'polysaccharide catabolic process'(G0:0000272) and the 'Starch and sucrose metabolism'(ko00500) pathway were significantly induced by drought stress. Related to carbohydrate synthase and starch synthase were induced. The expression level of sucrose synthase increased, and one of the genes encoding SUS7(RchiOBHm_Chr3g0488651) up-regulated 4.03-fold at DT3. Except for a small portion of starch synthase genes that were down-regulated, all were up-regulated. Additionally, drought treatment significantly induced the expression of enzyme genes involved in starch and sugar metabolism, fructose, and mannose metabolism. Among these, genes encoding fructose-1,6bisphosphatase, fructose-bisphosphate aldolase, mannose-1-phosphate guanyltransferase alpha, pyrophosphate-fructose 6-phosphate 1-phosphotransferase subunit beta-like and 6-phosphofructo-2kinase/fructose-2,6-bisphosphatase were down-regulated at DT3 (Additional file 9: Table S8).

In addition, we found that 89 DEGs associated with lipid metabolism were enriched in the lipid metabolic process'(GO:0006629), most of them related to the biosynthesis and metabolic processes of lipoprotein, wax, keratin, lipid, and fatty acid. Most genes involved in wax biosynthesis were significantly upregulated, for example, one gene encoding CYP86B1(RchiOBHm_Chr4g0432631) up-regulated 9.90-fold at DT3(Additional file 10: Table S9). Moreover, KEGG functional annotation of these differentially expressed genes related to metabolism. Many differentially expressed genes were related to secondary metabolisms. The total of 95 DEGs involved in secondary metabolism were enriched by KEGG function, 
and the results were shown in Fig.8. Drought stress affected the transcription level of genes related to phenylpropanoid biosynthesis pathways.

\section{Photosynthesis-related genes under drought stress}

We observed that many DEGs enriched in the category of 'photosynthesis'(ko00915) were overexpressed in DEGs down-regulated by DT2 and DT3, and in the process of DT2 and DT3, many genes involved in photosynthesis were significantly down-regulated. A total of $70 \mathrm{DEGs}$ involved in photosynthesis were identified. Among them, three (CAB40, LHCB5, psaA) of 16 DEGs encoding the components of photosystem I (PSI) were slightly up-regulated in DT1, and the other genes were significantly downregulated during the drought treatment period. A total of 33 genes encoding PS\& were identified, including 6 up-regulated genes ( $p s b E$, 3genes encoding $p s b C, p s b D, P S B 27-1)$ and 27 down-regulated genes. Most of these genes decreased significantly in the middle and late periods of drought, indicating that PSI and $\mathrm{PS} \otimes$ were suppressed under drought stress. Meanwhile, there were 15 genes encoding redox chains, all of which were significantly down-regulated in the middle and late periods of drought stress. Additionally, the expression of 6 genes encoding components of chloroplasts were significantly decreased (Additional file 11: Table S10).

\section{Transcription factors responding to drought stress}

Transcription factors specifically binding to cis-acting regulatory elements in the promoter of target genes are crucial regulatory proteins, which can modulate numbers of genes up or down-regulate [22]. In this study, WRKY (58), NAC (43), MYB (52), bZIP (19), bHLH(55) families contained large numbers of transcription factors. The expression level of the WRKYs family showed large changes under different treatments. The expression of some genes reached the maximum in the middle and late periods of drought. Genes encoding WRKY75 and WRKY71 were all reached the highest expression level at DT3, upregulated more than 5-fold. WRKY57 (RchiOBHm_Chr5g0083891) was up-regulated regarding to expression responses at different drought stress, especially increased 7.93-fold at DT3. However, over half of the WRKY family has decreased expression levels at different drought levels. Twenty-four of genes encoding WRKYS were down-regulated at DT1 by 1.02- to 3.71-fold (Fig.9c, Additional file 12: Table S11).

More than half of the genes encoding NACs are up-regulated to varying degrees under drought stress. One gene encoding NAC100 (RchiOBHm_Chr2g0167461) continued to increase in the middle and late periods of drought stress, especially by 7.20 -fold. A total of 11 genes upregulated during DT1. Some genes increased and then decreased under drought stress, but increased after rewatering. For example, NAC073 (RchiOBHm_Chr0c19g0500091) was upregulated 5.78-fold at DT1, decreasing slightly at DT2, then down-regulated 2.64-fold at DT3, but it was increased by 6.96-fold after rewatering. NAC037 (RchiOBHm_Chr2g0099671) has a similar expression trend, but the up-regulation decrease by a small 
amount. On the contrary, one gene encoding NACO72 (RchiOBHm_Chr5g0034761) was down-regulated at DT1 and continued to increase afterwards (Fig.9a, Additional file 12: Table S11.).

In addition, under different drought treatment, the expression level of more than three-quarters of the MYBs genes showed different degrees of up-regulation. Among them, the number of up-regulated genes that appeared during the DT1 period was relatively large. For instance, MYB46

(RchiOBHm_Chr1g0315931) and MYB61 (RchiOBHm_Chr1g0315931) showed the highest up-regulation of expression with 7.09 and 7.15-fold. Moreover, some genes were up-regulated significantly at DT3. MYB75 (RchiOBHm_Chr2g0116041) was the most up-regulated among all up-regulated genes at DT3, increased by 7.65 -fold. Some genes showed a tendency to increase and then decrease with drought treatment, such as gene encoding MYB61 (RchiOBHm_Chr3g0458721), slightly increased at DT1 and then decreased by 6.10 -fold. After rewatering, a small amount of gene expression was up-regulated, and most of the gene expression did not change significantly, comparing with CK (Fig.9d, Additional file 12: Table S11.).

In the three drought treatments of the $b H L H$ families, the number of genes up-regulated in one period was more than the number of genes down-regulated, and the number of down-regulated in two periods was more than that. Most genes of the $b H L H$ family were up-regulated during the early drought period, DT1, while large numbers of genes were significantly down-regulated during the middle and late drought periods (DT2 and DT3). Among these, there was a slight upward adjustment, up-regulated by 1.02- to 4.66-fold at DT1, but there were 37 genes down-regulated at DT2 or DT3. One gene encoding $b H L H 72$ (RchiOBHm_Chr7g0182341) was down-regulated 6.94-fold. A gene bHLH121

(RchiOBHm_Chr6g0245181) belonging to the $b H L H$ family was no significant change in gene expression during drought treatment, but it was up-regulated after rewatering (Fig.9b; Additional file 12: Table S11.).

Among $A P 2 / D R E B$ and $b Z I P$ transcription factors, more genes were down-regulated under drought treatment. Nineteen members of $b Z I P s$ were found to be responsive to drought stress and 12 were downregulated, from 1.24- to 4.80-fold (Fig.9e; Additional file 12: Table S11.). Five genes encoding DREBs, all down-regulated exceeded 6.60-fold at DT1. The most significant down-regulation was that the DREB1D (RchiOBHm_Chr7g0199351) was down-regulated 9.64-fold (Fig.9f; Additional file 12: Table S11.).

\section{Co-expression network construction and prediction of regulatory circuitry controlling genes in drought stress response}

Gene co-expression network gene clustering and module cutting combined genes with similar expression patterns on the same branch. Each branch represented a co-expression module and different colors represented different modules. A total of 18 modules were identified (Fig.10a). The module gene expression pattern in each sample was displayed with the module characteristic value (Fig.10c). Transcription factors are an important class of regulatory proteins in biological processes. This study 
analyzed the transcription factors in each module. Results showed that the number of transcription factors in the module was 2 (salmon 2)-286 (coral 2) (Fig.10b). There were differences in the distribution of transcription factors in different modules, but mainly concentrated in WRKY, NAC, MYB, ERF, bHLH, $b Z I P, C 2 H 2$ and other transcription factor families. According to previous reports, these transcription factors were involved in the process of plant stress regulation [23]. Furthermore, the module-trait correlation relationships are shown in Fig.11. Four interesting modules, coral 2, lightsteelblue 1, navajowhite 1 and bisque 4, were selected according to the criterion of $|r|>0.5$ and $P<0.05$. We analyzed the expression pattern of the co-expressed genes in these four selected modules (Fig.12). Meanwhile, further annotated these four modules with GO and KEGG analysis (Additional file 1: Figure S2; Additional file 1: Figure S3; Additional file 1: Figure S4). We found that in the navajowhite 1 module, the significantly rich GO terms indicated the key biological processes of co-expressed gens, and many genes were enriched in various stress responses. Mainly enriched in 'response to stimulus'(G0:0050896). 'response to chemical'(GO:0042221), 'response to water'(GO:0009415) and 'response to water deprivation'(G0:0009414) were also significantly enrich (Fig.13a). KEGG enrichment analysis showed that the main enrichment in metabolic pathways (Fig.13b). Therefore, we speculated that this module genes may play an important role in the process of rose drought stress and rewatering.

Weighted gene co-expression network analysis (WGCNA) was used to identify putative transcription factors. The transcription factors of the navajowhite 1 module were analyzed, and screened out the highly connected genes, which were used as the core genes. Highly connected nodes in expression networks were defined as hub genes. Many DEGs Identified in the navajowhite1 module were annotated as TFs, including several WRKY, MYB, NAC, ERF, ARF and bHLHTFs (Fig.14a). Based on the hub gene correlation network and their high connectivity, 22 of these TFs were particularly selected: two genes (RchiOBHm_Chr5g0034761 and RchiOBHm_Chr6g0308271) encoding NAC072, two genes (RchiOBHm_Chr2g0167461 and RchiOBHm_Chr7g0181811) encoding NAC100, NAC087 (RchiOBHm_Chr3g0497471), MYB330(RchiOBHm_Chr7g0228621), MYB75 (RchiOBHm_Chr2g0116041), MYB102 (RchiOBHm_Chr5g0006031), MYB78 (RchiOBHm_Chr4g0426181), WRKY3 (RchiOBHm_Chr4g0439041), WRKY4 (RchiOBHm_Chr2g0166991), WRKY6 (RchiOBHm_Chr5g0040801), WRKY27(RchiOBHm_Chr1g0378621), WRKY28 (RchiOBHm_Chr2g0151681), WRKY71 (RchiOBHm_Chr5g0013131), two genes (RchiOBHm_Chr4g0429851 and RchiOBHm_Chr2g0175911) encoding WRKY75, ERF113 (RchiOBHm_Chr4g0428891), ARF1 (RchiOBHm_Chr7g0219771), ARF5 (RchiOBHm_Chr6g0302551), ARF8 (RchiOBHm_Chr3g0487771) and the bHLH3 (RchiOBHm_Chr6g0288981) (Fig.14b).

\section{Validation of the DEGs by qRT-PCR}

To verify the accuracy and reproducibility of the transcriptome analysis, 13 selected DEGs were analyzed the transcript abundance using qRT-PCR, including 7 randomly selected transcription factors from navajowhite 1 module, another 4 transcription factors and 2 ABA synthesis-related DEGs. The results showed that the expression profiles detected by qRT-PCR were positively correlated with the RNA-Seq 
results (Fig.15). Therefore, the reliability of our RNA-seq data was confirmed by the consistency between the qRT-PCR results and RNA-seq analyses.

\section{Discussion}

\section{Transcription factors involved in the drought stress response}

TFs play important roles in various abiotic stress responses process which under the control of regulating downstream stress response genes [24]. During the signal transduction, TFs directly regulates the expression of related genes by acting as a molecular switch. These TFs interact specifically with ciselements located in the promoter region of their regulatory [25]. Numerous TFs were obtained in the transcriptome sequencing in this study including the NACs, WRKYs, MYBs, AP2/ERFs, bHLHs and so on. Through the analysis of WGCNA and transcription regulation modules, the highly connected TFs in the navajowhite 1 module were selected. Many studies have shown that NACs play positive roles in the regulation of plant stress resistance. Arabidopsis [26], wheat [27, 28], and rice [29, 30] have the most researches on NACs, which have found that overexpression of NACs transcription factor family genes can significantly enhance the tolerance of transgenic plants to multiple stresses. In addition, rose (Rosa hybrida) RhNAC3 can improve rose petals dehydration tolerance, and can also improve the drought tolerance of transgenic Arabidopsis [14]. In the study of rose (Rosa chinensis 'Slater's crimson China') heat resistance, it was found that both NAC27 and NAC72 were up-regulated [31]. NAC27 and NAC72 were also involved in ABA signal transduction under heat stress of Rhododendron hainanensis [32]. Overexpression of $N A C 72$ in Arabidopsis thaliana can increase the drought resistance of plants, and it also proved that it mediates the regulation of ABA-responsive genes [33]. We selected a gene encoding NAC072 with high connectivity in the navajowhite 1 module, indicating that it plays an important role in the regulation of rose drought stress. Combined with previous studies, we speculate that NACO72 may be involved in ABA signal transduction under drought stress in R.chinensis. Furthermore, the role of NAC087 and NAC100 in drought also needs to be concerned, which were also the core genes in the navajowhite 1 module.

The WRKY genes play pivotal roles in stress responses. Many researches have demonstrated that WRKYS have important biological functions in the response of plants to different kinds of biotic and abiotic stresses [34]. Studies on Arabidopsis, rice, and soybean have shown the role of the WRKYs in response to drought stress. For example, AtWRKY57 [35], AtABO3 [36], OsWRKY47 [37], and GmWRKY54 [38] all improve the drought tolerance of plants. Likewise, VaWRKY14 responds to drought and cold stress, and overexpression of VaWRKY14 enhances the drought tolerance of transgenic Arabidopsis [39]. However, some WRKYs can act as a negative regulator of abiotic stress in plants. For instance, GhWRKY33 [40] and SIWRKY81 [41, 42] reduce the drought tolerance of transgenic plants. In our analysis, eight WRKY genes including WRKY75, WRKY28 and WRKY27 were found to have high connectivity in the 
navajowhite1 module, indicating that they may be involved in drought response. Poplar research found that PagWRKY75 was down-regulated in the early stages of salt and osmotic stress, transgenic poplar lines overexpressing PagWRKY75 were more sensitive to salt and osmotic [43]. It can be assumed that WRKY 75 plays an important regulatory role in abiotic stress, but the function of WRKY75 in drought stress screened in this study needs further verification.

\section{Phytohormone signals under drought stress}

The phytohormone play a key role in abiotic stress responses and coordinate various signal transduction pathways. In this study, after drought treatment, the gene expression involved in several plant hormonerelated signal transduction pathways changed significantly, indicating that plant hormones may play an important role in the response of the rose to drought stress. ABA acts as an endogenous messenger in plants abiotic stress responses $[44,45]$. Drought results in a substantial increases of plant ABA levels, accompanied by a major change in gene expression and in adaptive physiological responses. NCED is a key rate-limiting enzyme in the ABA synthesis pathway $[46,47]$. Currently, some NCED3 genes have been isolated and identified, and corresponding gene function studies have shown that NCED3 genes play an important role in enhancing plant drought resistance [48-50]. We found that an NCED3 gene was upregulated by 2.70 -fold at DT1. Interestingly, the NCED6 gene was down-regulated by 9.40 -fold in DT3. Lefebvre et al. indicates that AtNCED6 is involved in ABA biosynthesis during seed development [51]. The role of NCED6 in drought stress needs further study. Another ABA synthesis related gene aldehyde oxidase $(A A O 3)$ and zeaxanthin cyclooxygenase (ZEP) were identified, both of which were significantly down-regulated in DT3. These results suggested that the synthesis of ABA may increase during the early drought and decrease during the late drought. The ABA core signaling pathway is highly conservative in evolution, for example, in maize and rice, they all follow the signal transduction process of PYR1/PYLs/RCARs-PP2C-SnRK2s [52]. Furthermore, we detected four PP2Cs and four PLYs, which are important components of the core signal network of ABA-mediated drought tolerance, the expression levels of three PP2Cs genes increased under different drought stress levels, one PP2C gene expression decreased during the late drought. However, PLYs genes expression decreased under drought stress. Generally, the transcription level of PYR1/PYLs/RCARs is down-regulated under stress conditions, but the abundance of $P P 2 C$ transcription increases [53]. Therefore, ABA-mediated signal transduction may be involved in the drought response of R.chinensis.

There are more and more evidences show that the phytohormone ethylene is essential for regulating various developmental processes and stress responses of plants [54, 55]. McMichael et al. reported that the release of ethylene may increase under drought conditions [56], while studies on pineapples have shown that drought-stressed plants produce significantly less ethylene in leaf and stem tissues compared to control plants [57]. These conflicting results may be due to the differences in different species. In general, the production of ethylene can be affected by changing of $A C C$ biosynthesis under drought stress [58]. In plants, ACC synthase (ACS) and ACC oxidase $(A C O)$ are two most important enzymes in ethylene biosynthetic pathway [59]. In our study, under drought stress, the expression of three ACS genes

Page $14 / 61$ 
was suppressed, the expression of one ACS gene was up-regulated, while the expression of two ACO genes was up-regulated. It is not yet possible to speculate whether the ethylene content has increased or decreased under drought conditions. Ethylene response factors (ERFs) are downstream components of the ethylene signaling pathway [60]. Experiments showed that ERFs play important roles in plant abiotic stress response [61, 62]. We have identified large number of ERFs, which had different expressions under different drought levels, and their role under drought stress needs further study.

In fact, all aspects of plant growth and development require the phytohormone auxin. Studies have shown that the response of auxin to plant drought stress mediates the regulatory network that exists between auxin signaling and drought stress $[63,64]$. Small auxin-upregulated RNAs (SAURs) as auxin rapid response genes play key role in auxin signaling pathway. Many SAURs respond to abiotic stresses, such as salt and drought, but they have different response patterns under abiotic stress $[65,66]$. In this study, two SAUR genes continued to be up-regulated, two SAUR genes continued to be down-regulated, one SAUR gene was up-regulated and then down-regulated. The function of the SAUR gene has been studied in wheat [67], and the function of the SAUR gene in the R.chinensis needs further study. The auxin response factors $(A R F S$ ) have been revealed to play crucial roles in regulating the expression of auxin response genes [68]. However, its role under drought stress is limited. We have identified four ARFs genes which expression changes were inconsistent during drought stress.

Jasmonates (JAs), a class of oxygenated lipid derivatives, considered to be phytohormones necessary for plant growth and environmental adaptation [69]. MYCs protein are one of JASMONATE-ZIM(JAZ) target proteins and a key transcription factor regulating the corresponding genes downstream of JA. According to reports, $M Y C 2, M Y C 3$ and $M Y C 4$ are the central nodes of the JA signal regulation [70]. Lipoxygenase $(\angle O X)$, allene oxide synthase $(A O S)$, allene oxide cyclase $(A O C)$ and oxophytodienoate reductase $(O P R)$ are important enzyme genes in JA synthesis pathway [71]. The study has shown that $R h H B 1$ binds to the RhLOX4 promoter to suppress its expression in the cut rose (Rosa hybrida), and dehydration tolerance decreases [72]. We found that these genes were identified and most genes were upregulated under drought stress. Other studies have shown that JA was observed to increase in Pinus pinaster [73] plants and Oryza sativa (rice) [74] leaves exposing to drought conditions. Based on these results, we speculated that the JA pathway plays a role in the response to drought stress in R.chinensis.

Moreover, recent studies have indicated that the role of brassinosteroid (BR) in plant response to drought stress [75-77]. In Norma Fàbregas's [78] study, the overexpression of $B R L 3$, a vascular-rich member of the BR family, can confer Arabidopsis drought resistance without compromising normal plant growth. The expression of two $B R L$ genes increased under drought stress in this study, we speculate that BRLs play positive parts in the response to drought. In addition, BR and ABA regulated the drought resistance of plants. Indeed, the molecular basis of the antagonistic effect of the BR-ABA pathway has been defined $[77,79]$, and the role of $A B A$ in promoting the response to drought stress suggests that BRs will inhibit drought stress responses. 


\section{Signaling pathway mediates drought-stress responses}

When plants are subjected to adversity stress owing to the external environment changed, plants senses adversity stimuli from outside, recognizes and transduces between cells and cells, and transmits them to downstream genes, thereby causing downstream effect gene expression to resist adversity. Calcium ions are called the second messengers of the cell, which mediates the calcium signal plays an important role in the plant response to external stimuli $[80,81]$. The three main families of calcium sensors in $\mathrm{Ca}^{2+}$ signaling in plants are calmodulin $(C a M)$, calcineurin B-like $(C B L)$ and calcium-dependent protein kinases (CDPKS) [82, 83]. Analysis of the CaM/CMLs identified in this study revealed that five genes were upregulated, two were up-regulated and then down-regulated, and interestingly fifteen were-down-regulated under drought stress. AtCML8, AtCML13, AtCML 18 and AtCML25 were down-regulated by salt damage and drought [84]. ShCML44 was up-regulated under cold, drought and salt damage [85]. In contrast, the gene encoding CML44 was down-regulated and the gene encoding $C M L 8$ was up-regulated under drought induction in this study. CDPKS/CPKs are involved in multiple stress signaling pathways. In Arabidopsis, Overexpressed AtCPK6 confers drought tolerance [86], while AtCPK21 is a negative regulator of osmotic response [87]. In this study, a total of six genes encoding putative $\mathrm{Ca}^{2+}$-dependent protein kinases were up-regulated during drought stress, and four of them showed high expression levels during the DT2 period. These results indicated that CDPK pathway had been significantly activated in DT2. CBLs communicate with downstream CIPKs for signal transmission. The CBL-CIPK pathways also participates in plant abiotic stress response [88]. In our current research, it is worth noting that three genes encoding $C B L s$-CIPKs were significantly down-regulated in DT3, which indicated that the CBL-CIPK pathway may contribute to regulate the late drought response in R.chinensis. In summary, the above results suggest that $\mathrm{Ca}^{2+}$ mediated signaling pathway has important functions in the response of $R$.chinensis to drought stress.

Plants sense and mediate various intracellular and extracellular signals through cell surface receptor kinases after stimulating by the external environment. Studies have shown that receptor-like protein kinases (RLKs) play an important role in plant growth and stress adaptation [89]. Leucine-rich repeat receptor protein kinases (LRR-RLKs), a class of single transmembrane proteins, are the largest family of plant receptor protein kinases [90]. The LRR-RLKs genes identified by us were 1 up-regulated and 10 down-regulated under drought stress, that was similar to the expression of $L R R-R L K s$ genes (3 upregulated, 38 down-regulated) in chrysanthemum under dehydration stress [6]. Additionally, multiple $M A P K s$ were observed during the plant response to abiotic stresses such as salt, drought, cold and heat, mainly rapid activation of $M P K 3, M P K 4$ and $M P K 6$ [91]. The results in this study are related to similarly, a gene encoding MPK3 was significantly down-regulated on DT1, indicating that MPK3 responded quickly when drought stress occurred.

MAPK cascade signals can also transmit reactive oxygen species (ROS) signals to downstream targets. Abiotic stress will cause plants to produce excessive reactive oxygen species, and reactive oxygen species will cause damage to plants. Plants have efficient enzymes including superoxide dismutase 
(SOD), Peroxidase (POD), ascorbate peroxidase (APX), glutathione peroxidase (GPX) and so on. The antioxidant defense mechanisms can protect plants from oxidative stress [92]. In this study, two genes encoding $A P X$ were up-regulated and four were down-regulated. Similarly, in the study of Citrullus lanatus, it was found that the changes of $A P X$ genes are different under drought stress [93]. The post-harvest petals of cut rose (Rosa hybrida cv. Samantha) under water deficit stress reported that the transcription level of RhAPX may be involved in the response of water deficit stress [94]. Interestingly, three genes encoding $S O D$ were identified, all of which were reduced under drought stress, while when we measured SOD activity founding that SOD activity increased as the degree of drought stress increased. The expression pattern of SOD genes in this study like this expression pattern of the SmFSD2 in drought. The SmFSD2 did not change significantly over $3 \mathrm{~d}$, but decreased to the lowest at $9 \mathrm{~d}$ [95]. These result indicate that the ROS signaling pathway may be involved in the regulation of $R$.chinensis response to drought stress.

\section{Metabolism in response to drought stress in R.chinensis}

Primary and secondary metabolites act as signaling molecules or protective agents when plants response to adversity stress. The primary metabolic processes were involved in the response to the drought stress of $R$.chinensis, producing a series of osmotic protective agents, such as sugars, starches, amino acids, and lipids. Accumulation of sugars in various plants is related to high tolerance to drought stress $[96,97]$. This result is consistent with what we observed. We found that most genes related to carbohydrate synthesis including fructose, glucose, mannose, sucrose, and trehalose were induced and significantly up-regulated under drought stress. Interestingly, the gene encoding trehalose phosphate synthase (TPP1) was down-regulated under drought stress. Of the 11 OsTPS genes in the rice (Oryza sativa) genome, only OsTPS1 has TPS activity [98]. Overexpression of OsTPS1 can improve the tolerance of rice seedlings to drought stress; at the same time, it also causes the expression of some stress-related genes to be up-regulated and the phenotype is not obvious change [99]. Trehalose may involve in the process in which rose resistance to drought. The expression levels of four genes encoding starch synthase were significantly reduced at DT3, and there was no significant change in expression levels in the remaining periods compared with the control. It is speculated that the starch content may decrease in the late drought.

Lipids are an important component of cells and organelles, and a series of genes which synthesize lipid biosynthesis have been identified in drought treated $R$. chinensis plants. Long-chain acyl-CoA synthetases (LACS) are indispensable in the pathway of lipids synthesis and degradation of higher plants. There are nine genes in the LACSs family in Arabidopsis, among which LACS1 and LACS2 are involved in the synthesis of wax and cutin together, and there is overlap in function [100,101]. Drought stress can cause rapid accumulation of wax on plant surface [102]. It is worth noting that six genes encoding LACS2 were induced under drought, and five of them were significantly down-regulated at the DT3 in this study. Studies on bananas, Agave sisalana [103] and Poa pratensis [104] have reported that waxy synthetic transcripts are significantly down-regulated under drought stress. These results confirm that wax may 
play key roles in regulating the drought resistance of $R$. chinensis, and speculate that the wax content may be reduced in the later period of drought stress. In addition, we found that the gene encoding GDSL esterase/lipase was significantly induced in the beginning of drought stress. Studies on the GDSL esterase/lipase gene of Arabidopsis thaliana revealed that this gene helps plants resist abiotic stress [105], and this gene is induced in M. wufengensis by cold stress [106]. Summarily, lipid metabolism is crucial for $R$. chinensis adaptation to drought stress.

In addition, as part of the adaptation mechanism to the environment of plants, secondary metabolism is sensitive to both biological and abiotic stresses [107]. It is known that the phenylpropane pathway has a high responsiveness to different abiotic stresses such as injury, drought and low or high temperature. White grapes (Vitis vinifera L.) cape with drought by stimulating the phenylpropane pathway, that is like our findings. We found that most of the secondary metabolites are derived from phenylpropane biosynthesis and flavonoid biosynthesis pathways. We have identified genes which encoding phenylalanine ammonia lyase (PAL) and coumaric acid-CoA ligase ( $4 C L)$, they are all related enzyme genes in the phenylpropane metabolism pathway. They were significantly up-regulated at DT1, presuming that the phenylpropane metabolism pathway was induced under drought stress. The transcription of $P A L$ of Caragana korshinskii is increased under field and laboratory drought conditions [108]. These results also confirm our results. Another major derivative of the phenylpropane pathway is flavonoids, which play a role in adversity stress. Flavonoids with free radical scavenging activity alleviate oxidative and drought stress in Arabidopsis thaliana [109]. We reported that genes encoding flavonoid synthase (FLS), chalcone isomerase $(C H S)$ and anthocyanin synthase $(A N S)$ were induced under drought. Flavonoid products may have an effect on the regulation of $R$. chinensis by drought stress.

\section{Conclusions}

Here we reported the drought transcriptome study of R.chinensis, with the purpose of understanding the mechanism of its drought response and identifying functional genes related to drought tolerance.

According to the transcriptome data, the model of R.chinensis drought response is summarized in Fig. 16, signal receptors, such as ion channel proteins sense external drought stimuli and are transmitted through

$\mathrm{Ca}^{2+}, \mathrm{ROS}$ and phytohormone signaling transduction. Signal transmission is through protein kinases and protein phosphorylases which can activate transcription factors. The activation of TFs triggers downstream drought-responsive gene transcription such as lipid metabolism, carbohydrate metabolism and secondary metabolism to regulate cell homeostasis. We selected candidate genes and transcription factors involved in the $\mathrm{Ca}^{2+}, \mathrm{ROS}, \mathrm{ABA}$ and ethylene signaling pathways as well as secondary metabolism, which may help us to further study the response mechanism of $R$.chinensis drought stress.

\section{Methods}

\section{Plant material and treatments}


Two-year-old healthy cutting seedlings of R.chinensis used in these experiments were collected from Beijing Institute of Landscape Architecture (Beijing, China). The plant material was planted in the pot with an inner size of $26 \mathrm{~cm}$ in diameter, $22 \mathrm{~cm}$ in height, and $18 \mathrm{~cm}$ in diameter at the bottom. It contains a soil with a mixture of nutritive soil, garden soil, perlite and vermiculite in a ratio of 5:3:1:1(v/v). The size and height of plant materials used in all experiments were almost the same. Before drought stress, the plant samples were cultured under the following growth conditions: $50 \%$ relative humidity, $25^{\circ} \mathrm{C} / 18{ }^{\circ} \mathrm{C}$, day/night temperatures in the same artificial climate chamber of Beijing Forestry University (BJFU) (116.3 ${ }^{\circ} \mathrm{E}, 40.0^{\circ} \mathrm{N}$ ). Afterwards, these plant samples were subjected to continuously no-watering for $15 \mathrm{~d}, 30 \mathrm{~d}$, $45 \mathrm{~d}, 60 \mathrm{~d}, 75 \mathrm{~d}, 90 \mathrm{~d}$ and rehydration treatment, the fully watered as a control. Reference to experimental methods of drought treatment from Samuel David Gamboa-Tuz [110]. At each time point, the fourth or fifth fully expanded leaves from the plants were collected, then immediately frozen in liquid nitrogen and stored at $-80{ }^{\circ} \mathrm{C}$ until physiological measurement or RNA extraction. Each treatment group contained three replicates, and each sample from 3 replicates to obtain biological replicates.

\section{Measurement of physiological parameters}

The relative water content of leaves, superoxide dismutase (SOD) activity, malondialdehyde (MDA) and relative soil water content were evaluated before transcriptome sequencing using leaf tissue which was collected and stored at $-80^{\circ} \mathrm{C}$ from plants subjected to 0 to 90 days of drought stress.

Relative soil water content (RSWC) was analyzed using direct drying method, weighing the empty aluminum box and recorded as M0 before taking an appropriate amount of soil with a depth of about 6 $\mathrm{cm}$ around the toot system of the plant, weighing it in an aluminum box (M1),putting it in an oven at 105 ${ }^{\circ} \mathrm{C}$ for $12 \mathrm{~h}$, and weighing it after cooled to room temperature in a desiccator (M2) , then RSWC was gotten by equations:

$\operatorname{RSWC}(\%)=(\mathrm{M} 1-\mathrm{M} 2) /(\mathrm{M} 1-\mathrm{M} 0) * 100 \%$

The relative water content of leaves was determined related to the initial fresh weight of the leaf samples, the samples which were soaked in distilled water were placed on the lab bench with dark cloth for $24 \mathrm{~h}$ and then weighted, reference from Rong Zhou et al with slight modifications [111]. The thiobarbituric acid (TBA) reaction was used for evaluating the MDA content as described by Dhindsa et al [112] with minor adjustments. The SOD activity was measured following the spectrophotometer by determining its ability to inhibit the photochemical reduction of nitro blue tetrazolium (NBT). Each experiment included three biological replicates.

\section{RNA isolation, cDNA library preparation and transcriptome sequencing}


Total RNA was isolated with an Easy Spin Plus RNA Extraction Kit (RN53, Aidlab China) following the manufacturer's instructions. RNA integrity was verified using electrophoresis on $1 \%$ agarose gel. The 2100 Bioanalyzer (Agilent Technologies, United States) and ND-2000 (NanoDrop Technologies) were employed to test the quality and purity of RNA.

The RNA-Seq library was generated using the NEB\#7530 RNA Library Prep Kit (\#E7530, New England Biolabs) following manufacturer's instructions. And then total mixed RNA from R.chinensis leaves in the control $(0 \mathrm{~d})$ and after $30 \mathrm{~d}, 60 \mathrm{~d}, 90 \mathrm{~d}$ of drought treatment, as well as, the rehydration treatment were used for cDNA library construction. The library quality was subsequently detected through the High Sensitivity DNA assay Kit (Agilent Technologies), and three biological replicates which from each experiment as previously described were sequenced using an Illumina HiSeqTM 4000 platform by Genedenovo Biotechnology, Co., Ltd. (Guangzhou, China).

\section{Mapping to the rose genome and gene expression quantification}

HISAT2.2.4 software [113] was used to carry out comparative analysis based on the rose genome [10]. According to the results of HISAT2 comparison StringTie [114, 115], it was used to reconstruct the transcript, and the expression of all genes in each sample was calculated.

\section{Identification of differentially expressed genes (DEGs) and genes co-expression network analysis}

The Fragments Per Kilobase of Exon Per Million Mapped Fragments (FPKM) method was used to analysis the expression level of each transcript. The mapped reads of each sample were assembled by StringTie v1.3.1 in a reference-based approach. For each transcription region, a FPKM value was calculated by StringTie software to quantify its expression abundance and variations. Differential expression analysis was performed by DESeq2 software [116] between two different groups (also by edgeR [117] between two samples). The genes with the parameter of false discovery rate (FDR) below 0.05 and absolute fold change $\geq 2$ was considered differentially expressed genes (DEGs). Gene Ontology (GO) enrichment analyses of DEGs [118] was able to recognize the main biological functions. Next, KEGG [119] pathway enrichment analysis identified significantly enriched metabolic pathways or signal transduction pathways in DEGs.

Co-expression networks were established using Weighted Gene Co-expression Network Analysis (WGCNA) (v1.47) package in R [120]. Gene expression values were imported into WGCNA to construct co-expression modules using the qutomatic network construction function blockwiseModules with default settings, except that the power is 10 , TOMType is unsigned, minModuleSize is 50 . Using module eigengenes to calculate the correlation coefficient with samples, which find out significant modules. The networks were 
visualized using Cytoscape_3.3.0 [121], then, GO and KEGG enrichment analyses were conducted for genes in each module. The calculated p-value was gone through FDR and correction taking FDR $\leq 0.05$ as a threshold.

\section{Real-time quantitative PCR verification}

Total RNAs were isolated from leaves of the treatment and controlled as described above. First-strand cDNA synthesis was performed using Prime Script $\triangle 1$ st strand cDNA Synthesis Kit (Takara, Shiga, Japan) according to the manufacturer instructions. The qRT-PCR was performed using a Bio-Rad/CFX ConnectTM Real-Time PCR Detection System (Bio-Rad, CA, USA) with SYBR® qPCR mix (Takara, Shiga, Japan) according to the manufacturer's protocol. Relative mRNA content was calculated using the $2^{-\triangle \triangle \mathrm{Ct}}$ method against the internal reference gene RcPP2A [122]. The primers used in this study were designed with Primer Premier 5 and are listed in Additional file 12: Table S11. Three biological replicates were performed for all reactions.

\section{Statistical analysis}

Data of physiological responses were analyzed using SPSS, and means were compared using the least significant difference (LSD) at $\mathrm{P}=0.05$.

\section{Abbreviations}

GO: Gene ontology; KEGG: Kyoto Encyclopedia of Genes and Genomes Pathway; NCBI: National Center for Biotechnology Information; qRT-PCR: Real-time quantitative reverse transcription polymerase chain reaction; WGCNA: Weighted Gene Co-expression Network Analysis; SOD: Superoxide dismutase; MDA: Malondialdehyde; RSWC: Relative soil water content; TBA: Thiobarbituric acid; DEGs: Differentially expressed genes; TFs: Transcription factors

\section{Declarations}

\section{Authors' contributions}

$\mathrm{YL}, \mathrm{SZ}, \mathrm{XJ}$ and HF conceived and designed the research. XJ performed the experiments, analyzed the data, and wrote the manuscript. YB and NJ cultivated and provided the rose plants. All authors have read and agreed to the published version of the manuscript.

\section{Funding}

This research was funded by Beijing Municipal Science and Technology Project (z191100008519005) and Chongming District, Shanghai Agricultural Science and Technology Innovation Project (2019CNKC- 
03).

\section{Availability of data and materials}

\section{Ethics approval and consent to participate}

Not applicable.

\section{Consent for publication}

Not applicable.

\section{Competing interests}

The authors declare that they have no competing interests.

\section{References}

1. Zhu JK. Salt and drought stress signal transduction in plants. Annu Rev Plant Biol. 2002;53:247-73.

2. Chaves MM, Maroco JP, Pereira JS. Understanding plant responses to drought - From genes to the whole plant. Functional Plant Biology. 2003;30(3).

3. Valliyodan B, Nguyen HT. Understanding regulatory networks and engineering for enhanced drought tolerance in plants. Curr Opin Plant Biol. 2006;9(2):189-95.

4. Huang X, Hou L, Meng J, You H, Li Z, Gong Z, et al. The Antagonistic Action of Abscisic Acid and Cytokinin Signaling Mediates Drought Stress Response in Arabidopsis. Mol Plant. 2018;11(7):97082.

5. Liu C, Mao B, Ou S, Wang W, Liu L, Wu Y, et al. Correction to: OsbZIP71, a bZIP transcription factor, confers salinity and drought tolerance in rice. Plant Mol Biol. 2018;97(4-5):467-8.

6. Xu YJ, Gao S, Yang YJ, Huang MY, Cheng LN, Wei Q, et al. Transcriptome sequencing and whole genome expression profiling of chrysanthemum under dehydration stress. Bmc Genomics. 2013;14.

7. Zhao DQ, Zhang XY, Fang ZW, Wu YQ, Tao J. Physiological and Transcriptomic Analysis of Tree Peony (Paeonia section Moutan DC.) in Response to Drought Stress. Forests. 2019;10(2).

8. Zhou A, Ma H, Liu E, Jiang T, Shuang F, Gong S, et al. Transcriptome Sequencing of Dianthus spiculifolius and Analysis of the Genes Involved in Responses to Combined Cold and Drought Stress. International Journal of Molecular Sciences. 2017;18(4):849.

9. Dai F, Zhang C, Jiang X, Kang M, Yin X, Lu P, et al. RhNAC2 and RhEXPA4 Are Involved in the Regulation of Dehydration Tolerance during the Expansion of Rose Petals. Plant Physiology. 
2012;160(4):2064-82.

10. Raymond O, Gouzy J, Just J, Badouin H, Verdenaud M, Lemainque A, et al. The Rosa genome provides new insights into the domestication of modern roses. Nat Genet. 2018;50(6):772-7.

11. Zhang X, Lu SC, Jiang CH, Wang YF, Lv B, Shen JB, et al. RcLEA, a late embryogenesis abundant protein gene isolated from Rosa chinensis, confers tolerance to Escherichia coli and Arabidopsis thaliana and stabilizes enzyme activity under diverse stresses. Plant Molecular Biology. 2014;85(45):333-47.

12. Li ZY, Chen W, Zhang C, Du CX, Shao GY, Cui YY, et al. RcMYBPA2 of Rosa chinensis functions in proanthocyanidin biosynthesis and enhances abiotic stress tolerance in transgenic tobacco. Plant Cell Tissue and Organ Culture. 2019;137(3):441-54.

13. Jiang X, Li S, Ding A, Zhang Z, Hao Q, Wang K, et al. The Novel Rose MYB Transcription Factor RhMYB96 Enhances Salt Tolerance in Transgenic Arabidopsis. Plant Molecular Biology Reporter. 2018;36(3):406-17.

14. Jiang XQ, Zhang CQ, Lu PT, Jiang GM, Liu XW, Dai FW, et al. RhNAC3, a stress- associated NAC transcription factor, has a role in dehydration tolerance through regulating osmotic stress- related genes in rose petals. Plant Biotechnology Journal. 2014;12(1):38-48.

15. Ding A, Li S, Li W, Hao Q, Wan X, Wang K, et al. RhNAC31, a novel rose NAC transcription factor, enhances tolerance to multiple abiotic stresses in Arabidopsis. Acta Physiologiae Plantarum. 2019;41(6).

16. Debener T, Linde M. Exploring Complex Ornamental Genomes: The Rose as a Model Plant. Critical Reviews in Plant Sciences. 2009;28(4):267-80.

17. İşeri ÖD, Körpe DA, Sahin FI, Haberal M. Hydrogen peroxide pretreatment of roots enhanced oxidative stress response of tomato under cold stress. Acta Physiologiae Plantarum. 2013;35(6):1905-13.

18. Kovtun, Yelena, Chiu, Wan-Ling. Functional analysis of oxidative stress-activated mitogen-activated protein kinase cascade in plants. Proceedings of the National Academy of Sciences of the United States of America. 2000.

19. Shinozaki K, Yamaguchi-Shinozaki K, Seki M. Regulatory network of gene expression in the drought and cold stress responses. Current Opinion in Plant Biology. 2003;6(5):410.

20. Xiong L, Schumaker KS, Zhu JK. Cell Signaling during Cold, Drought, and Salt Stress. Plant Cell. 2002;14 Suppl(Suppl):S165-83.

21. Jaspers $P$, Kangasj?Rvi J. Reactive oxygen species in abiotic stress signaling. Physiologia Plantarum. 2010;138(4):405-13.

22. Dhriti S, Ashverya L. Transcriptional regulation of drought response: a tortuous network of transcriptional factors. Frontiers in Plant Science. 2015;6(895).

23. Erpen, Devi, HS, Grosser, JW, Dutt. Potential use of the DREB/ERF, MYB, NAC and WRKY transcription factors to improve abiotic and biotic stress in transgenic plants. Plant Cell Tiss Org. 2018. 
24. Joshi R, Wani SH, Singh B, Bohra A, Dar ZA, Lone AA, et al. Transcription Factors and Plants Response to Drought Stress: Current Understanding and Future Directions. Front Plant Sci. 2016;7:1029.

25. Franco-Zorrilla JM, Lopez-Vidriero I, Carrasco JL, Godoy M, Vera P, Solano R. DNA-binding specificities of plant transcription factors and their potential to define target genes. Proceedings of the National Academy of Sciences of the United States of America. 2014;111(6):2367-72.

26. Tran LS, Nakashima K, Sakuma Y, Simpson SD, Fujita Y, Maruyama K, et al. Isolation and functional analysis of Arabidopsis stress-inducible NAC transcription factors that bind to a drought-responsive cis-element in the early responsive to dehydration stress 1 promoter. Plant Cell. 2004;16(9):2481-98.

27. Mao X, Chen S, Li A, Zhai C, Jing R, Turgay U. Novel NAC Transcription Factor TaNAC67 Confers Enhanced Multi-Abiotic Stress Tolerances in Arabidopsis. Plos One. 2014;9(1):e84359-.

28. Zhang L, Zhang L, Xia C, Zhao G, Jia J, Kong X. The Novel Wheat Transcription Factor TaNAC47 Enhances Multiple Abiotic Stress Tolerances in Transgenic Plants. Front Plant Sci. 2015;6:1174.

29. Hong $Y$, Zhang H, Lei H, Li D, Song F. Overexpression of a stress-responsive NAC transcription factor gene ONAC022 improves drought and salt tolerance in rice. Frontiers in Plant Science. 2016;7(e0116646):4.

30. Sung SJ, Nuri O, Joong CP, Shic KY, Do CY, Ju-Kon K. Overexpression of OsNAC14 Improves Drought Tolerance in Rice. Frontiers in Plant Science. 2018;9:310-.

31. Li ZQ, Xing W, Luo P, Zhang FJ, Jin XL, Zhang MH. Comparative transcriptome analysis of Rosa chinensis 'Slaters' crimson China' provides insights into the crucial factors and signaling pathways in heat stress response. Plant Physiology and Biochemistry. 2019;142:312-31.

32. Zhao, Ying, Yu, Wengang, Hu, Xiangyu, et al. Physiological and transcriptomic analysis revealed the involvement of crucial factors in heat stress response of Rhododendron hainanense. Gene An International Journal Focusing on Gene Cloning \& Gene Structure \& Function. 2018.

33. Li X, Li X, Li M, Yan Y, Xu L, Ling L. Dual function of NAC072 in ABF3-mediated ABA-responsive gene regulation in Arabidopsis. Frontiers in Plant Science. 2016;7.

34. Jiang, JJ, Ma, SH, Ye, NH, et al. WRKY transcription factors in plant responses to stresses. J Integr Plant Biol. 2017.

35. Jiang Y, Liang G, Yu D. Activated expression of WRKY57 confers drought tolerance in Arabidopsis. Mol Plant. 2012;5(6):1375-88.

36. Ren X, Chen Z, Liu Y, Zhang H, Zhang M, Liu Q, et al. ABO3, a WRKY transcription factor, mediates plant responses to abscisic acid and drought tolerance in Arabidopsis. Plant Journal for Cell \& Molecular Biology. 2010;63(3):417-29.

37. Raineri J, Wang S, Peleg Z, Blumwald E, Chan RL. The rice transcription factor OsWRKY47 is a positive regulator of the response to water deficit stress. Plant Molecular Biology. 2015;88(4):401-13.

38. Wei W, Liang De, Bian X, Shen M, Zhang Jo. Gm WRKY 54 improves drought tolerance through activating genes in $\mathrm{ABA}$ and $\mathrm{Ca} 2+$ signaling pathways in transgenic soybean. The Plant Journal. 2019;100(2). 
39. Langlang, Zhang, Jun, Cheng, Xiaoming, Sun, et al. Overexpression of VaWRKY14 increases drought tolerance in Arabidopsis by modulating the expression of stress-related genes. Plant cell reports. 2018.

40. Wang N-N, Xu S-W, Sun Y-L, Liu D, Zhou L, Li Y, et al. The cotton WRKY transcription factor (GhWRKY33) reduces transgenic Arabidopsis resistance to drought stress. Scientific Reports. 2019;9(1).

41. Li X, Wan H, Zhou G, Cheng Y, Ahammed GJ. SIWRKY81 reduces drought tolerance by attenuating proline biosynthesis in tomato. Scientia Horticulturae. 2020;270:109444.

42. Ahammed GJ, Li X, Yang Y, Liu C, Zhou G, Wan H, et al. Tomato WRKY81 acts as a negative regulator for drought tolerance by modulating guard cell H2O2-mediated stomatal closure. Environmental and Experimental Botany. 2020;171.

43. Zhao K, Zhang D, Lv K, Zhang X, Jiang T. Functional characterization of poplar WRKY75 in salt and osmotic tolerance. Plant ence. 2019;289:110259.

44. Cutler SR, Rodriguez PL, Finkelstein RR, Abrams SR. Abscisic Acid: Emergence of a Core Signaling Network. Annual Review of Plant Biology. 2010;61(1):651-79.

45. Teng K, Li J, Liu L, Han Y, Du Y, Zhang J, et al. Exogenous ABA induces drought tolerance in upland rice: the role of chloroplast and $A B A$ biosynthesis-related gene expression on photosystem II during PEG stress. Acta Physiologiae Plantarum. 2014;36(8):2219-27.

46. Schwartz SH, Tan BC, Gage DA, Zeevaart JA, McCarty DR. Specific oxidative cleavage of carotenoids by VP14 of maize. Science. 1997;276(5320):1872-4.

47. luchi S. Regulation of drought tolerance by gene manipulation of 9-cis-epoxycarotenoid dioxygenase, a key enzyme in abscisic acid biosynthesis in Arabidopsis (vol 27, pg 325, 2001). The Plant Journal. 2002;30(5):611-.

48. Hwang SG, Chen HC, Huang WY, Chu YC, Shii CT, Cheng WH. Ectopic expression of rice OsNCED3 in Arabidopsis increases ABA level and alters leaf morphology. Plant Science. 2010;178(1):0-22.

49. Qin X, Zeevaart JA. Overexpression of a 9-cis-epoxycarotenoid dioxygenase gene in Nicotiana plumbaginifolia increases abscisic acid and phaseic acid levels and enhances drought tolerance. Plant Physiol. 2002;128(2):544-51.

50. Li T, Sun J, Li C, Lu Z, Xia J. Cloning and expression analysis of the FvNCED3 gene and its promoter from ash (Fraxinus velutina). Journal of Forestry Research. 2019;30(2):471-82.

51. Lefebvre V, North H, Frey A, Sotta B, Seo M, Okamoto M, et al. Functional analysis of Arabidopsis NCED6 and NCED9 genes indicates that ABA synthesized in the endosperm is involved in the induction of seed dormancy. Plant J. 2006;45(3):309-19.

52. Wang YG, Fu FL, Yu HQ, Hu T, Zhang YY, Tao Y, et al. Interaction network of core ABA signaling components in maize. Plant Mol Biol. 2018;96(3):245-63.

53. Szostkiewicz I, Richter K, Kepka M, Demmel S, Ma Y, Korte A, et al. Closely related receptor complexes differ in their ABA selectivity and sensitivity. Plant J. 2010;61(1):25-35. 
54. Achard P, Cheng H, De Grauwe L, Decat J, Schoutteten H, Moritz T, et al. Integration of plant responses to environmentally activated phytohormonal signals. Science. 2006;311(5757):91-4.

55. Pei $\mathrm{H}$, Wang $\mathrm{H}$, Wang L, Zheng F, Dong $\mathrm{CH}$. Regulatory Function of Ethylene in Plant Responses to Drought, Cold, and Salt Stresses: John Wiley \& Sons, Inc.; 2017.

56. Mcmichael BL, Jordan WR, Powell RD. An Effect of Water Stress on Ethylene Production by Intact Cotton Petioles. Plant Physiology. 1972;49(4):658-60.

57. Min XJ, Bartholomew DP. Effects of flooding and drought on ethylene metabolism, titratable acidity and fruiting of pineapple. Acta Horticulturae. 2005;666(666):135-48.

58. Larrainzar E, Molenaar JA, Wienkoop S, Gil-Quintana E, Alibert B, Limami AM, et al. Drought stress provokes the down-regulation of methionine and ethylene biosynthesis pathways in Medicago truncatula roots and nodules. Plant Cell \& Environment. 2014;37(9).

59. Kende H. Enzymes of Ethylene Biosynthesis. Plant Physiology. 1989;91(1):1-4.

60. Hongwei, Guo, and, Joseph, Ecker. The ethylene signaling pathway: new insights. Current Opinion in Plant Biology. 2004.

61. Pan Y, Seymour GB, Lu C, Hu Z, Chen X, Chen G. An ethylene response factor (ERF5) promoting adaptation to drought and salt tolerance in tomato. Plant Cell Reports. 2012;31(2):349-60.

62. Yu Y, Yang D, Zhou S, Gu J, Wang F, Dong J, et al. The ethylene response factor OsERF109 negatively affects ethylene biosynthesis and drought tolerance in rice. Protoplasma. 2017;254(1):401-8.

63. Naser V, Shani E. Auxin response under osmotic stress. Plant Molecular Biology. 2016;91(6):661-72.

64. Shi H, Chen L, Ye T, Liu X, Ding K, Chan Z. Modulation of auxin content in Arabidopsis confers improved drought stress resistance. Plant Physiology \& Biochemistry. 2014;82:209-17.

65. Wang J, An C, Guo H, Yang X, Chen J, Zong J, et al. Physiological and transcriptomic analyses reveal the mechanisms underlying the salt tolerance of Zoysia japonica Steud. BMC Plant Biol. 2020;20(1):114.

66. Wang M, He X, Jiang B, Liu W, Lin Ye, Xie D, et al. Transcriptome analysis in different chieh-qua cultivars provides new insights into drought-stress response. Plant Biotechnology Reports. 2019;13(6):663-75.

67. GUO Y, XU C-b, SUN X-j, HU Z, ZHANG H. TaSAUR78 enhances multiple abiotic stress tolerance by regulating the interacting gene TaVDAC1. Journal of Integrative Agriculture. 2019;18(12):2682-90.

68. Li SB, Xie ZZ, Hu CG, Zhang JZ. A review of auxin response factors (ARF) in plants. Frontiers in Plant Science. 2016;7(742):47.

69. Wasternack C, Hause B. Jasmonates: biosynthesis, perception, signal transduction and action in plant stress response, growth and development. An update to the 2007 review in Annals of Botany. Ann Bot. 2013(6):1021-58.

70. Chico JM, Lechner E, Fernandez-Barbero G, Canibano E, Garcia-Casado G, Franco-Zorrilla JM, et al. CUL3(BPM) E3 ubiquitin ligases regulate MYC2, MYC3, and MYC4 stability and JA responses. Proc Natl Acad Sci U S A. 2020;117(11):6205-15. 
71. Devoto A, Turner JG. Jasmonate-regulated Arabidopsis stress signalling network. Physiologia Plantarum. 2005;123(2):161-72.

72. Fan Y, Liu J, Zou J, Zhang X, Jiang L, Liu K, et al. The RhHB1/RhLOX4 module affects the dehydration tolerance of rose flowers (Rosa hybrida) by fine-tuning jasmonic acid levels. Hortic Res. 2020;7:74.

73. Pedranzani H, Sierra-De-Grado R, Vigliocco A, Miersch O, Abdala G. Cold and water stresses produce changes in endogenous jasmonates in two populations of Pinus pinaster Ait. Plant Growth Regulation. 2007;52(2):111-6.

74. Du H, Liu H, Xiong L. Endogenous auxin and jasmonic acid levels are differentially modulated by abiotic stresses in rice. Front Plant Sci. 2013;4:397.

75. Nolan T, Chen J, Yin Y. Cross-talk of Brassinosteroid signaling in controlling growth and stress responses. Biochem J. 2017;474(16):2641-61.

76. Nolan, TM, Brennan, Yang, MR, Chen, et al. Selective Autophagy of BES1 Mediated by DSK2 Balances Plant Growth and Survival. Dev Cell. 2017.

77. Nolan TM, Vukasinovic N, Liu D, Russinova E, Yin Y. Brassinosteroids: Multidimensional Regulators of Plant Growth, Development, and Stress Responses. Plant Cell. 2020;32(2):295-318.

78. Fabregas N, Lozano-Elena F, Blasco-Escamez D, Tohge T, Martinez-Andujar C, Albacete A, et al. Overexpression of the vascular brassinosteroid receptor BRL3 confers drought resistance without penalizing plant growth. Nat Commun. 2018;9(1):4680.

79. Wang H, Tang J, Liu J, Hu J, Liu J, Chen Y, et al. Abscisic Acid Signaling Inhibits Brassinosteroid Signaling through Dampening the Dephosphorylation of BIN2 by ABI1 and ABI2. Mol Plant. 2018;11(2):315-25.

80. Kudla J, Batistic O, Hashimoto K. Calcium signals: the lead currency of plant information processing. Plant Cell. 2010;22(3):541-63.

81. Reddy ASN, Ali GS, Celesnik H, Day IS. Coping with Stresses: Roles of Calcium- and Calcium/Calmodulin-Regulated Gene Expression. Plant Cell. 2011;23(6):2010-32.

82. Ranty B, Aldon D, Galaud JP. Plant Calmodulins and Calmodulin-Related Proteins: Multifaceted Relays to Decode Calcium Signals. Plant Signaling \& Behavior. 2006;1(3):96-104.

83. Marie, Boudsocq, Jen, Sheen. CDPKs in immune and stress signaling. Trends Plant Sci. 2013.

84. McCormack E, Tsai YC, Braam J. Handling calcium signaling: Arabidopsis CaMs and CMLs. Trends Plant Sci. 2005;10(8):383-9.

85. Munir S, Liu H, Xing Y, Hussain S, Ouyang B, Zhang Y, et al. Overexpression of calmodulin-like (ShCML44) stress-responsive gene from Solanum habrochaites enhances tolerance to multiple abiotic stresses. Scientific Reports. 2016;6:31772.

86. Xu J, Tian YS, Peng RH, Xiong AS, Zhu B, Jin XF, et al. AtCPK6, a functionally redundant and positive regulator involved in salt/drought stress tolerance in Arabidopsis. Planta. 2010;231(6):p.1251-60. 
87. Franz S, Ehlert B, Liese A, Kurth J, Cazale AC, Romeis T. Calcium-dependent protein kinase CPK21 functions in abiotic stress response in Arabidopsis thaliana. Mol Plant. 2011;4(1):83-96.

88. Xiang $Y$, Huang $Y$, Xiong L. Characterization of stress-responsive CIPK genes in rice for stress tolerance improvement. Plant Physiol. 2007;144(3):1416-28.

89. Huachun, Wang, David, Chevalier, Clayton, Larue, et al. The Protein Phosphatases and Protein Kinases of Arabidopsis thaliana. Arabidopsis Book. 2007.

90. Gou X, He K, Yang H, Yuan T, Lin H, Clouse SD, et al. Genome-wide cloning and sequence analysis of leucine-rich repeat receptor-like protein kinase genes in Arabidopsis thaliana. Bmc Genomics. 2010;11.

91. Zhu, Jian-Kang. Abiotic Stress Signaling and Responses in Plants. Cell. 2016;167(2):313-24.

92. Gill SS, Tuteja N. Reactive oxygen species and antioxidant machinery in abiotic stress tolerance in crop plants. Plant Physiol Biochem. 2010;48(12):909-30.

93. Goitseone M, Hisashi T, Kinya A. The cDNA Structures and Expression Profile of the Ascorbate Peroxidase Gene Family During Drought Stress in Wild Watermelon. Journal of Agricultural Science. 2018;10(8):56-.

94. Jin J, Shan N, Ma N, Bai J, Gao J. Regulation of ascorbate peroxidase at the transcript level is involved in tolerance to postharvest water deficit stress in the cut rose (Rosa hybrida L.) cv. Samantha. Postharvest Biology \& Technology. 2006;40(3):236-43.

95. Shafi A, Gill T, Zahoor I, Ahuja PS, Sreenivasulu Y, Kumar S, et al. Ectopic expression of SOD and APX genes in Arabidopsis alters metabolic pools and genes related to secondary cell wall cellulose biosynthesis and improve salt tolerance. Molecular Biology Reports. 2019.

96. Bohnert HJ, Jensen RG. Strategies for engineering water-stress tolerance in plants. Trends in Biotechnology. 1996;14(3):89-97.

97. Ali Q, Shahid S, Ali S, Javed MT, Haider MZ. Trehalose Metabolism in Plants under Abiotic Stresses: Profiling and CounterAction2019.

98. Zang B, Li H, Li W, Deng XW, Wang X. Analysis of trehalose-6-phosphate synthase (TPS) gene family suggests the formation of TPS complexes in rice. Plant Molecular Biology. 2011;76(6):507-22.

99. Li HW, Zang BS, Deng XW, Wang XP. Overexpression of the trehalose-6-phosphate synthase gene OsTPS1 enhances abiotic stress tolerance in rice. Planta. 2011;234(5):p.1007-18.

100. Lü S, Song T, Kosma DK, Parsons EP, Rowland O, Jenks MA. Arabidopsis CER8 encodes LONG-CHAIN ACYL-COA SYNTHETASE 1 (LACS1) that has overlapping functions with LACS2 in plant wax and cutin synthesis. The Plant Journal. 2009.

101. Weng H, Molina I, Shockey J, Browse J. Organ fusion and defective cuticle function in a lacs 1 lacs2 double mutant of Arabidopsis. Planta. 2010;231(5):p.1089-100.

102. Kosma DK, Bourdenx B, Bernard A, Parsons EP, Lu S, Joubes J, et al. The Impact of Water Deficiency on Leaf Cuticle Lipids of Arabidopsis. Plant Physiology. 2009;151(4):1918-29. 
103. Sarwar MB, Ahmad Z, Rashid B, Hassan S, Gregersen PL, Leyva MDIO, et al. De novo assembly of Agave sisalana transcriptome in response to drought stress provides insight into the tolerance mechanisms. Scientific Reports. 2019;9(1).

104. Ni Y, Guo N, Zhao Q, Guo Y. Identification of candidate genes involved in wax deposition in Poa pratensis by RNA-seq. BMC Genomics. 2016;17:314.

105. Lai CP, Huang LM, Chen LFO, Chan MT, Shaw JF. Genome-wide analysis of GDSL-type esterases/lipases in Arabidopsis. Plant Molecular Biology. 2017.

106. Deng S, Ma J, Zhang L, Chen F, Sang Z, Jia Z, et al. De novo transcriptome sequencing and gene expression profiling of Magnolia wufengensis in response to cold stress. BMC Plant Biol. 2019;19(1):321.

107. Hartmann T. From waste products to ecochemicals: fifty years research of plant secondary metabolism. Phytochemistry. 2007;68(22-24):2831-46.

108. Liu F, Xie L, Yao Z, Zhou Y, Gong C. Caragana korshinskii phenylalanine ammonialyase is upregulated in the phenylpropanoid biosynthesis pathway in response to drought stress. Biotechnology \& Biotechnological Equipment. 2019;33(1):842-54.

109. Nakabayashi R, Yonekura-Sakakibara K, Urano K, Suzuki M, Yamada Y, Nishizawa T, et al. Enhancement of oxidative and drought tolerance in Arabidopsis by overaccumulation of antioxidant flavonoids. Plant Journal. 2014;77(3):367-79.

110. David Gamboa-Tuz S, Pereira-Santana A, Alejandro Zamora-Briseno J, Castano E, Espadas-Gil F, Tonatiuh Ayala-Sumuano J, et al. Transcriptomics and co-expression networks reveal tissue-specific responses and regulatory hubs under mild and severe drought in papaya (Carica papaya L.). Scientific Reports. 2018;8.

111. Zhou R, Yu X, Zhao T, Ottosen CO, Rosenqvist E, Wu Z. Physiological analysis and transcriptome sequencing reveal the effects of combined cold and drought on tomato leaf. BMC Plant Biol. 2019;19(1):377.

112. Heath RL, Packer K. Leaf senescense: Correlated with increased levels of membrane permeability and lipid peroxidation, and decreased levels of superoxide dismutase and catalase. Journal of Experimental Botany. 1968;32:93-101.

113. Kim D, Langmead B, Salzberg SL. HISAT: a fast spliced aligner with low memory requirements. Nature Methods. 2015;12(4):357-60.

114. Pertea M, Pertea GM, Antonescu CM, Chang TC, Mendell JT, Salzberg SL. StringTie enables improved reconstruction of a transcriptome from RNA-seq reads. Nature Biotechnology. 2015;33(3):290-5.

115. Pertea M, Kim D, Pertea GM, Leek JT, Salzberg SL. Transcript-level expression analysis of RNA-seq experiments with HISAT, StringTie and Ballgown. Nature Protocols. 2016;11(9):1650-67.

116. Love MI, Huber W, Anders S. Moderated estimation of fold change and dispersion for RNA-seq data with DESeq2. Genome Biol. 2014;15(12):550.

117. Robinson MD, McCarthy DJ, Smyth GK. edgeR: a Bioconductor package for differential expression analysis of digital gene expression data. Bioinformatics. 2010;26(1):139-40. 
118. Ashburner, Michael, Ball, Catherine, A., Blake, et al. Gene Ontology: tool for the unification of biology. Nature Genetics. 2000.

119. Ogata, Goto, Sato, Fujibuchi, Bono. KEGG: Kyoto Encyclopedia of Genes and Genomes. Nucleic Acids Research. 1999.

120. Langfelder P, Horvath S. WGCNA: an R package for weighted correlation network analysis. BMC Bioinformatics. 2008;9(1).

121. Shannon P, Markiel A, Ozier O, Baliga NS, Wang JT, Ramage D, et al. Cytoscape: a software environment for integrated models of biomolecular interaction networks. Genome Res. 2003;13(11):2498-504.

122. Klie M, Debener T. Identification of superior reference genes for data normalisation of expression studies via quantitative PCR in hybrid roses (Rosa hybrida). BMC research notes. 2011;4:518.

\section{Figures}

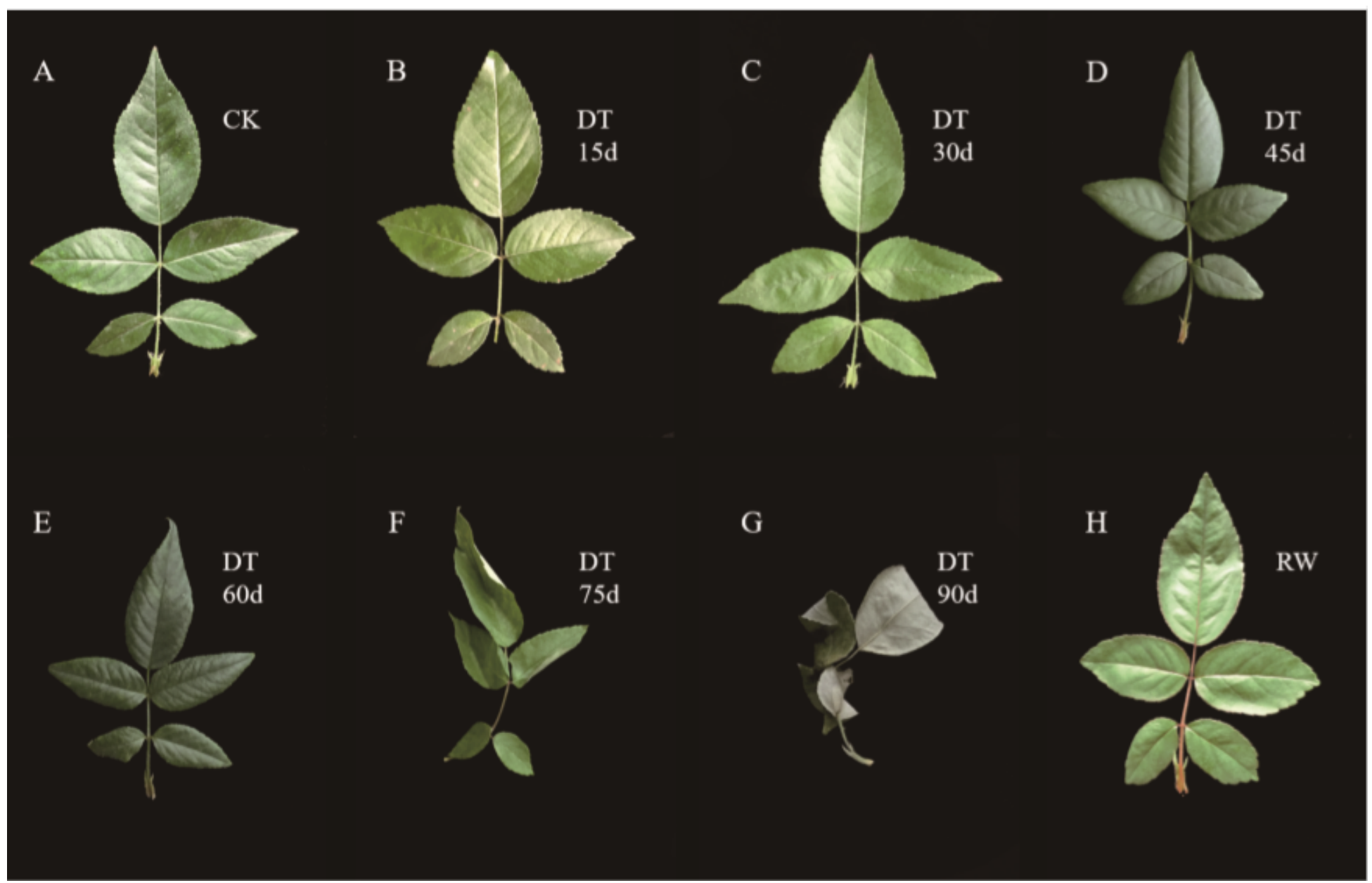

\section{Figure 1}

Leaf phenotypes of Rosa chinensis 'Old Blush' leaves during drought and rewatering stages. (A) The control plants. (B) Drought treatment for 15 days. (C) Drought treatment for 30 days. (D) Drought 
treatment for 45 days. (E) Drought treatment for 60 days. (F) Drought treatment for 75 days. (G) Drought treatment for 90 days. $(\mathrm{H})$ Rewatering treatment.

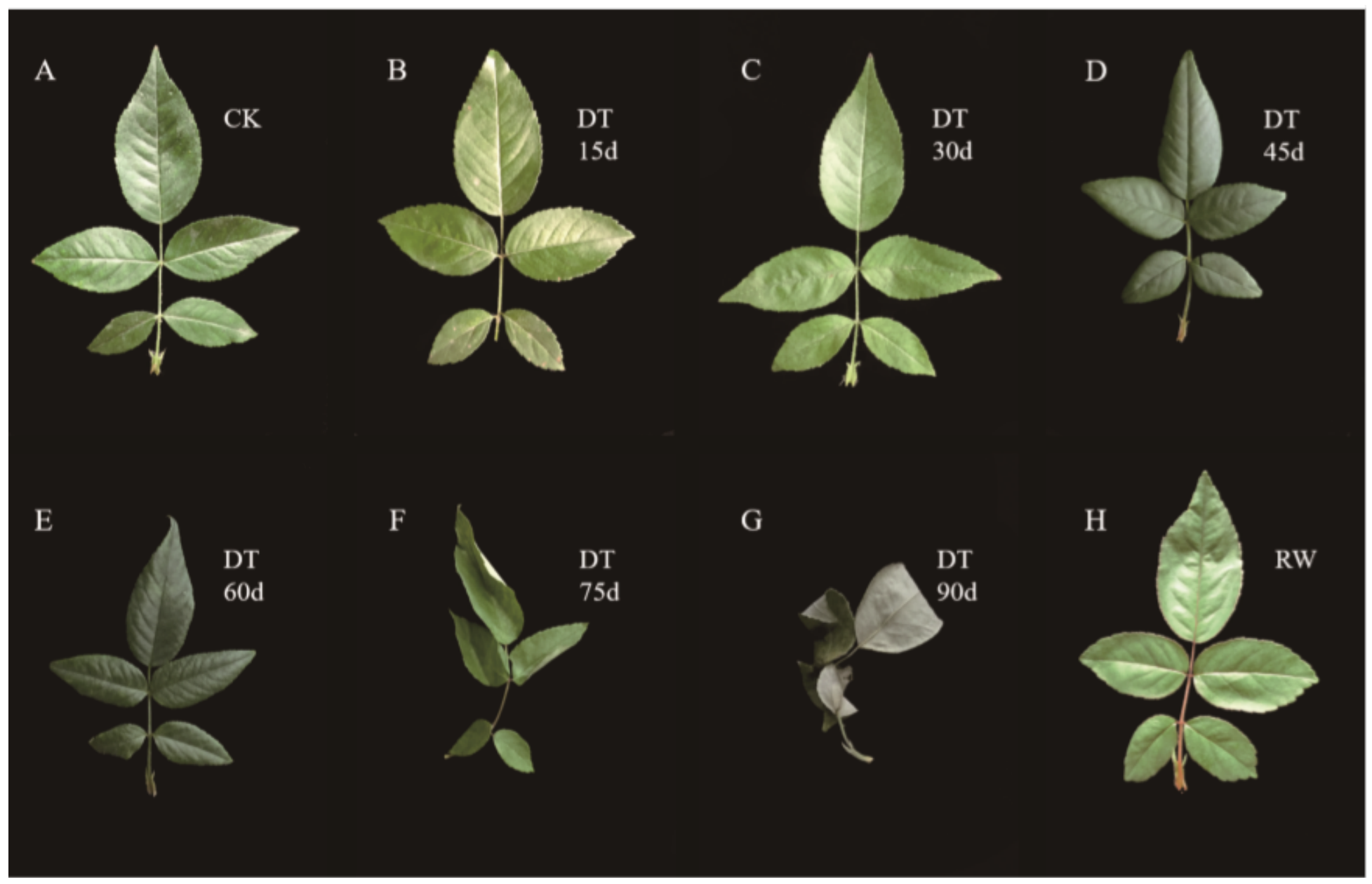

\section{Figure 1}

Leaf phenotypes of Rosa chinensis 'Old Blush' leaves during drought and rewatering stages. (A) The control plants. (B) Drought treatment for 15 days. (C) Drought treatment for 30 days. (D) Drought treatment for 45 days. (E) Drought treatment for 60 days. (F) Drought treatment for 75 days. (G) Drought treatment for 90 days. $(\mathrm{H})$ Rewatering treatment. 
A

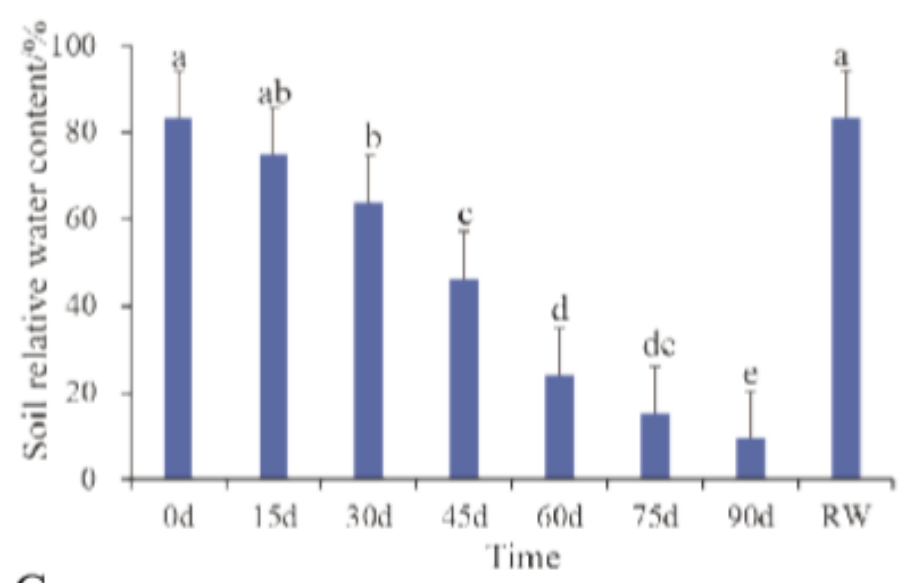

$\mathrm{C}$

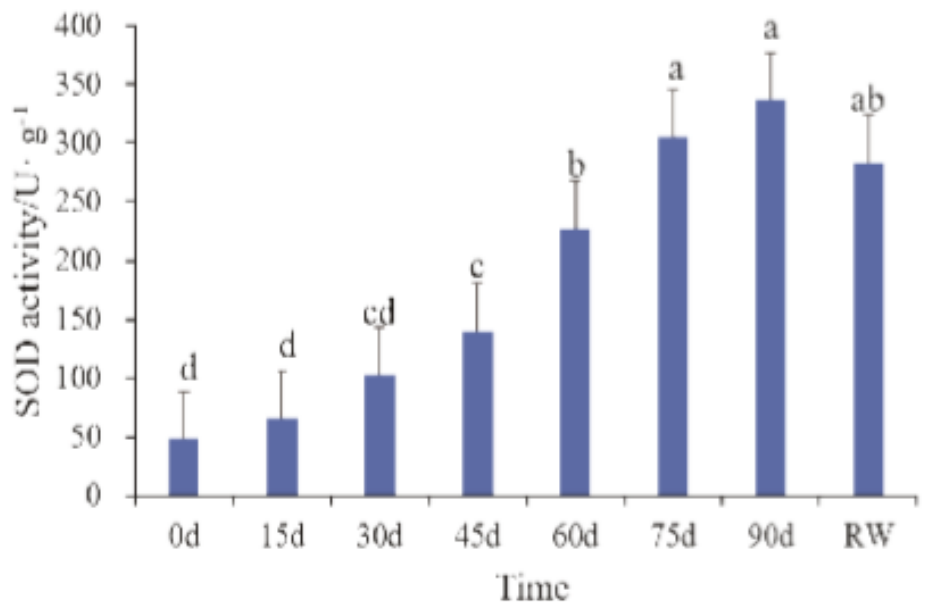

B

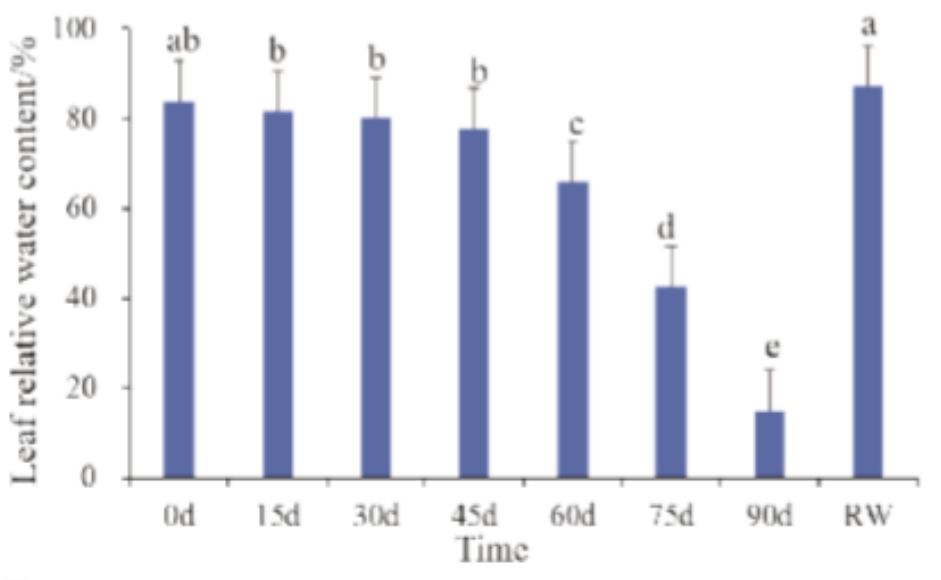

$\mathrm{D}$

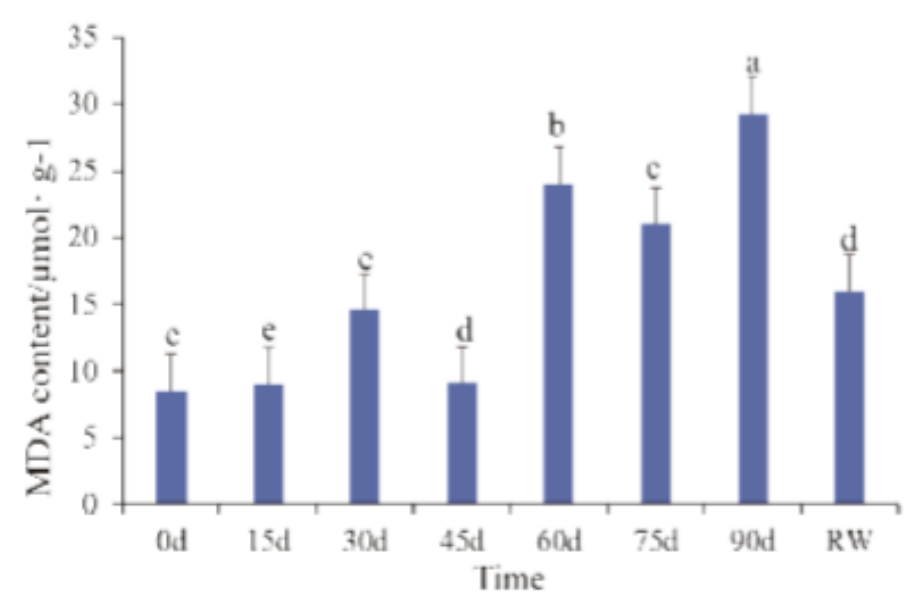

Figure 2

Physiological and biochemical changes of Rosa chinensis 'Old Blush' under drought and rewatering treatment. (A) Soil relative water content. (B) Leaf relative water content. (C) MDA content. (D) SOD activity. 
A

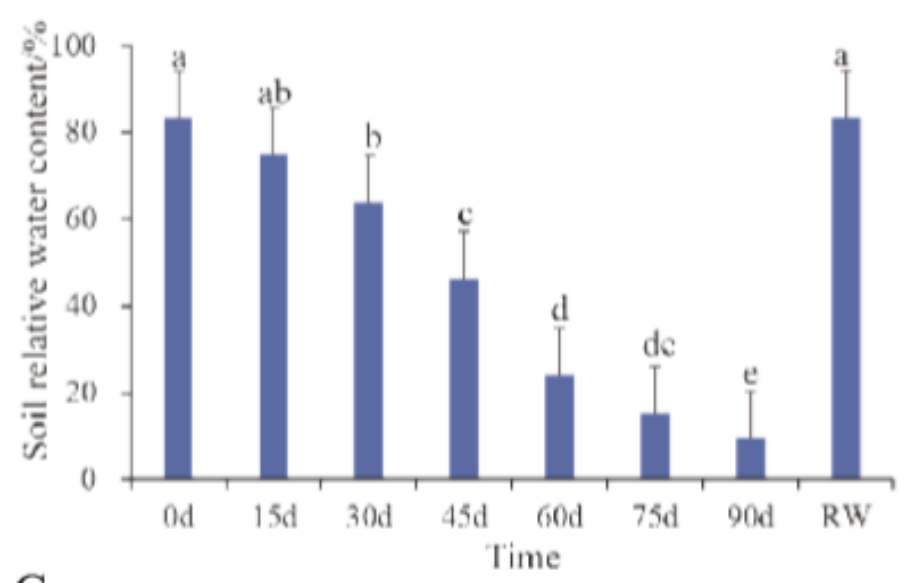

$\mathrm{C}$

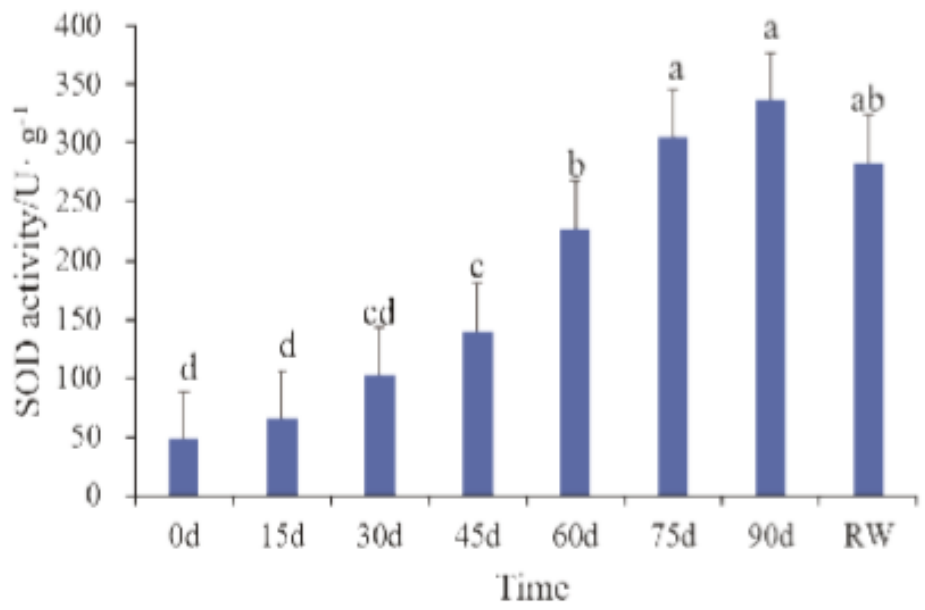

B

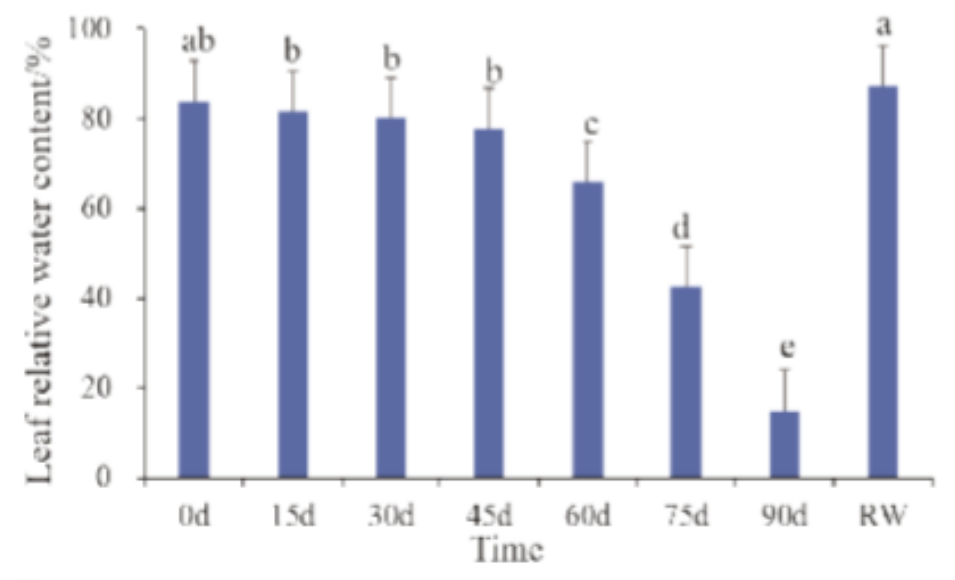

$\mathrm{D}$

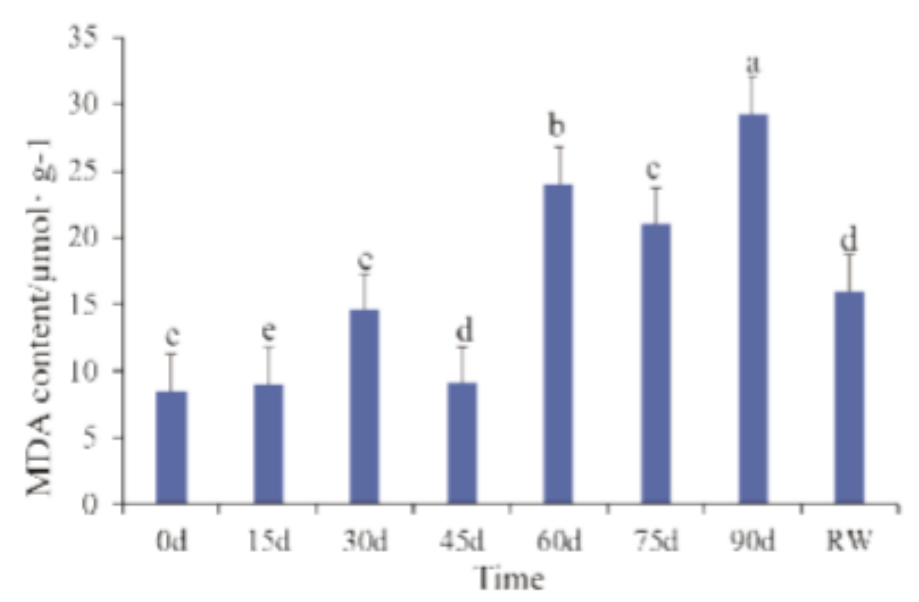

Figure 2

Physiological and biochemical changes of Rosa chinensis 'Old Blush' under drought and rewatering treatment. (A) Soil relative water content. (B) Leaf relative water content. (C) MDA content. (D) SOD activity. 
A

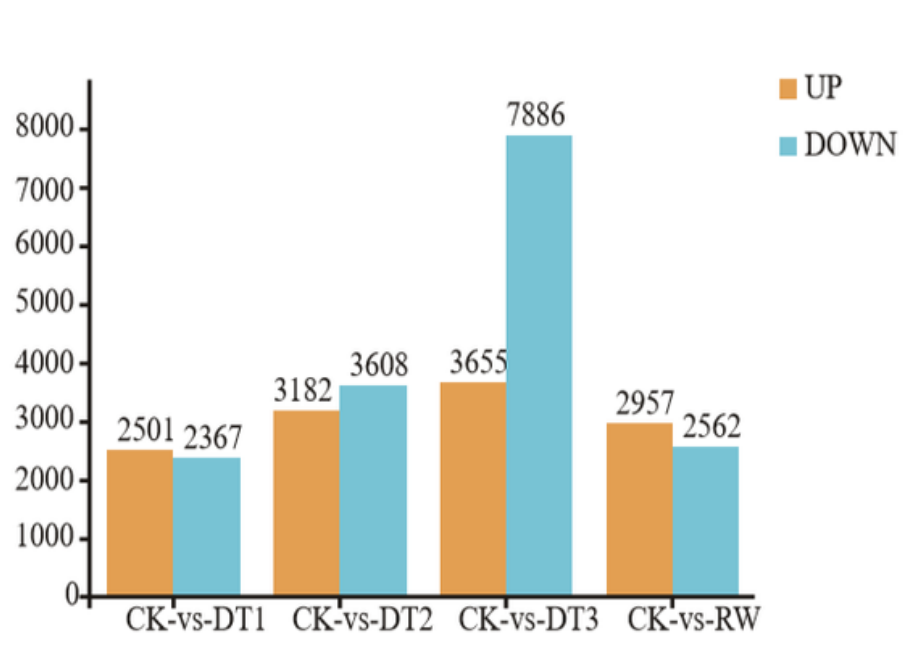

$\mathrm{B}$

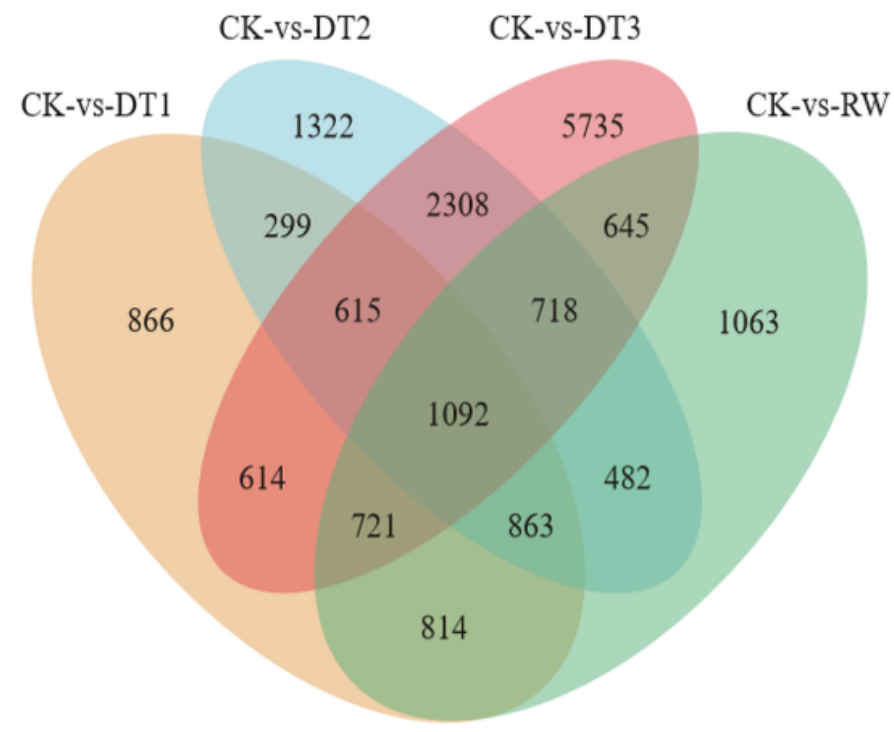

\section{Figure 3}

RNA-seq data expression profiles during drought and rewatering treatment in Rosa chinensis 'Old Blush'. (A) Veen diagram analysis of the DEGs from four drought and rewatering treatment stages compared with control plants. (B) The number of genes up-regulated and down-regulated in four drought and rewatering treatment stages compared with the control. Genes up-and down-regulated are shown yellow and blue bars.

A

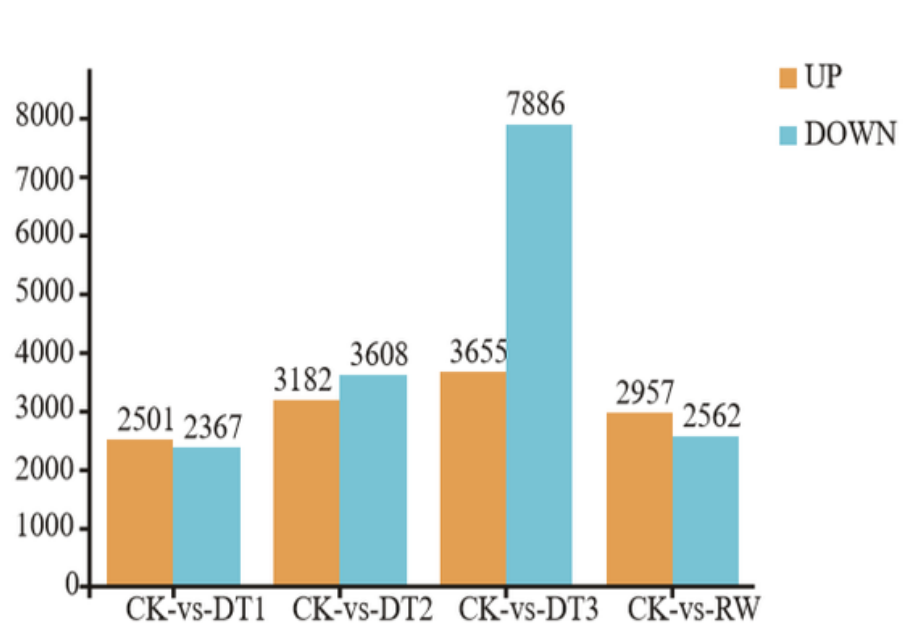

B

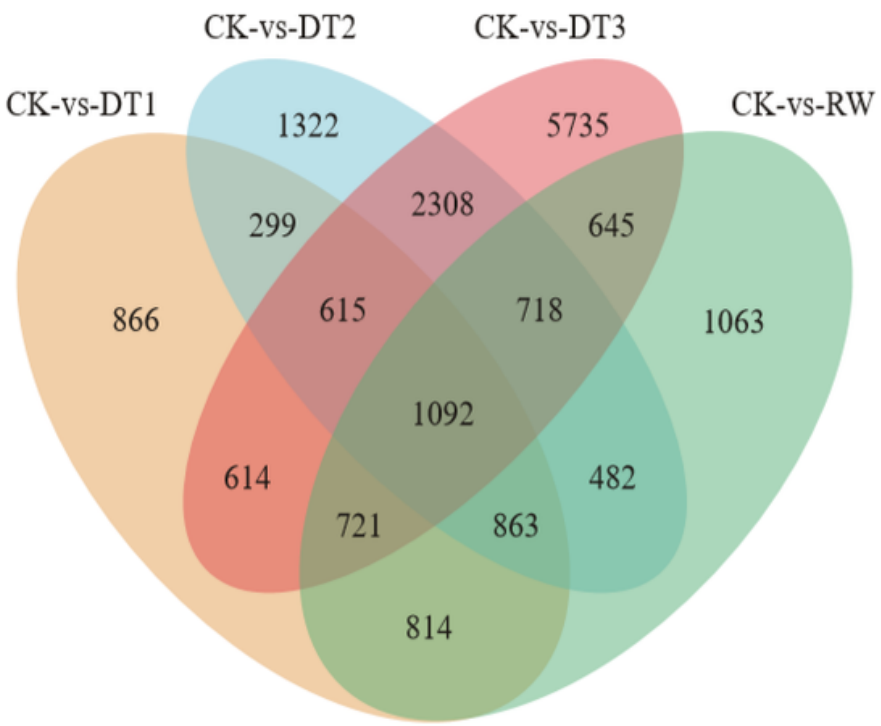

\section{Figure 3}

RNA-seq data expression profiles during drought and rewatering treatment in Rosa chinensis 'Old Blush'. (A) Veen diagram analysis of the DEGs from four drought and rewatering treatment stages compared 
with control plants. (B) The number of genes up-regulated and down-regulated in four drought and rewatering treatment stages compared with the control. Genes up-and down-regulated are shown yellow and blue bars.

A

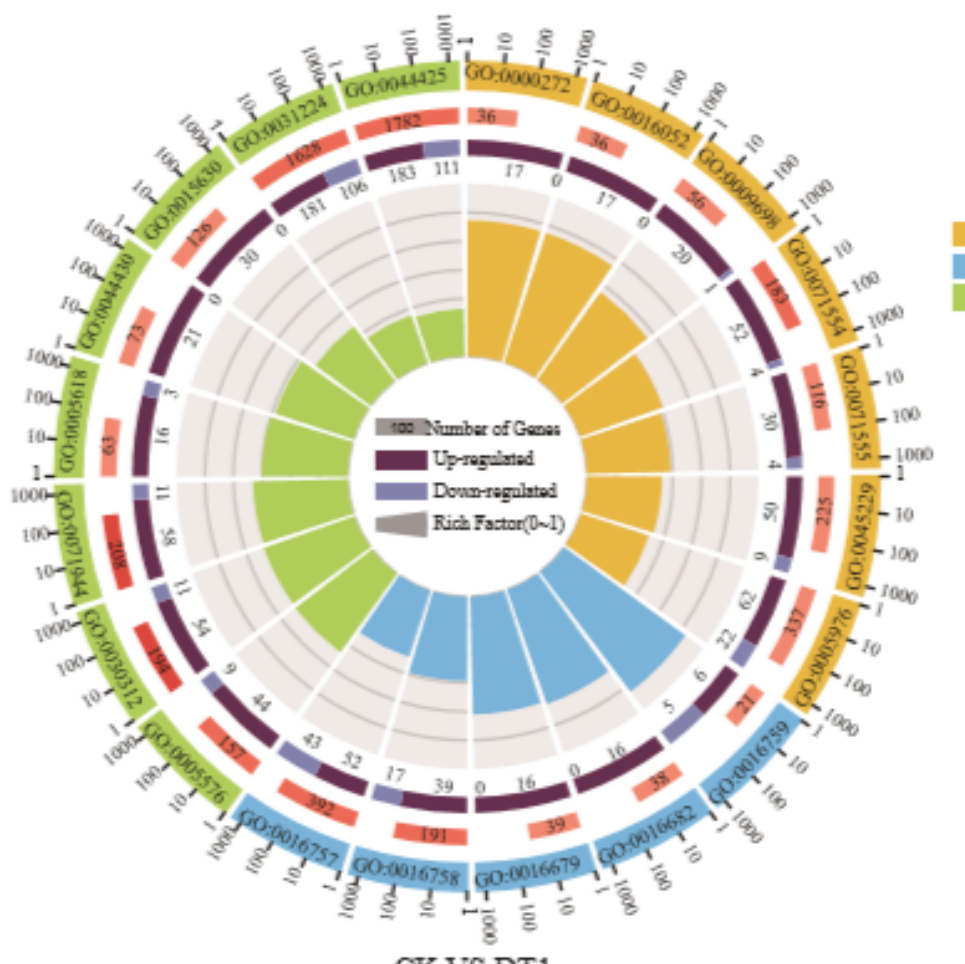

$$
\begin{aligned}
& \text { Biological Process } \\
& \text { Molecular Function } \\
& \text { Cellular Component } \\
& \\
& \text {-log10(Qvalue) } \\
& (0,1.3] \\
& (1.3,2] \\
& (2,5] \\
& (5,10] \\
& (10,15] \\
& (15,20] \\
& >=20
\end{aligned}
$$

B

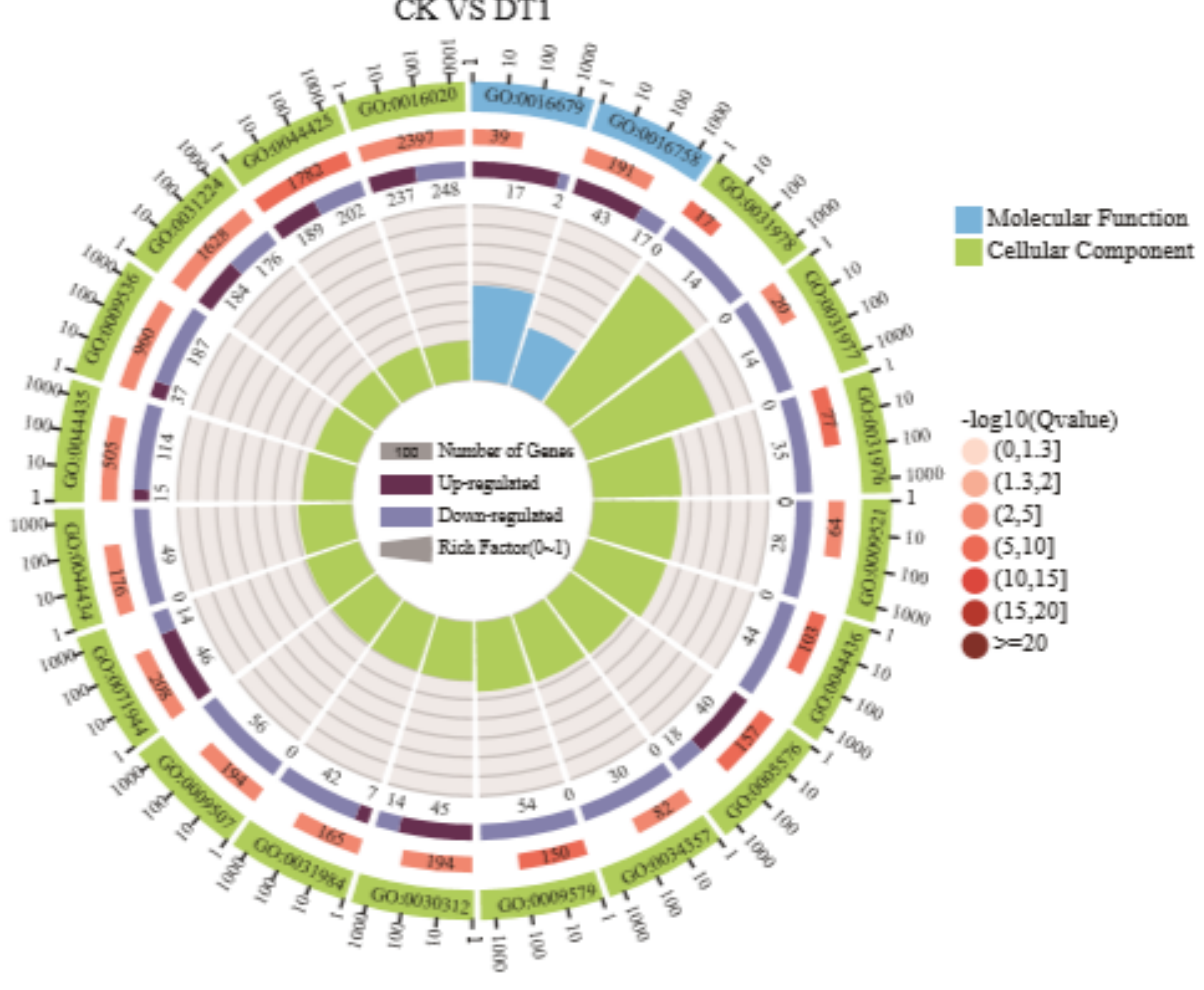

CK VS DT2

\section{Figure 4}

GO term analysis of control group and different treatment groups. (A) GO term analysis of CK vs DT1. (B) GO term analysis of CK vs DT2. The second lap represents the number of the genes in the genome 
background and the $Q$ values. The third lap represents the ratio of the upregulated genes (deep purple) and downregulated genes (light purple). The fourth lap represents the enrichment factor of each GO term.

A

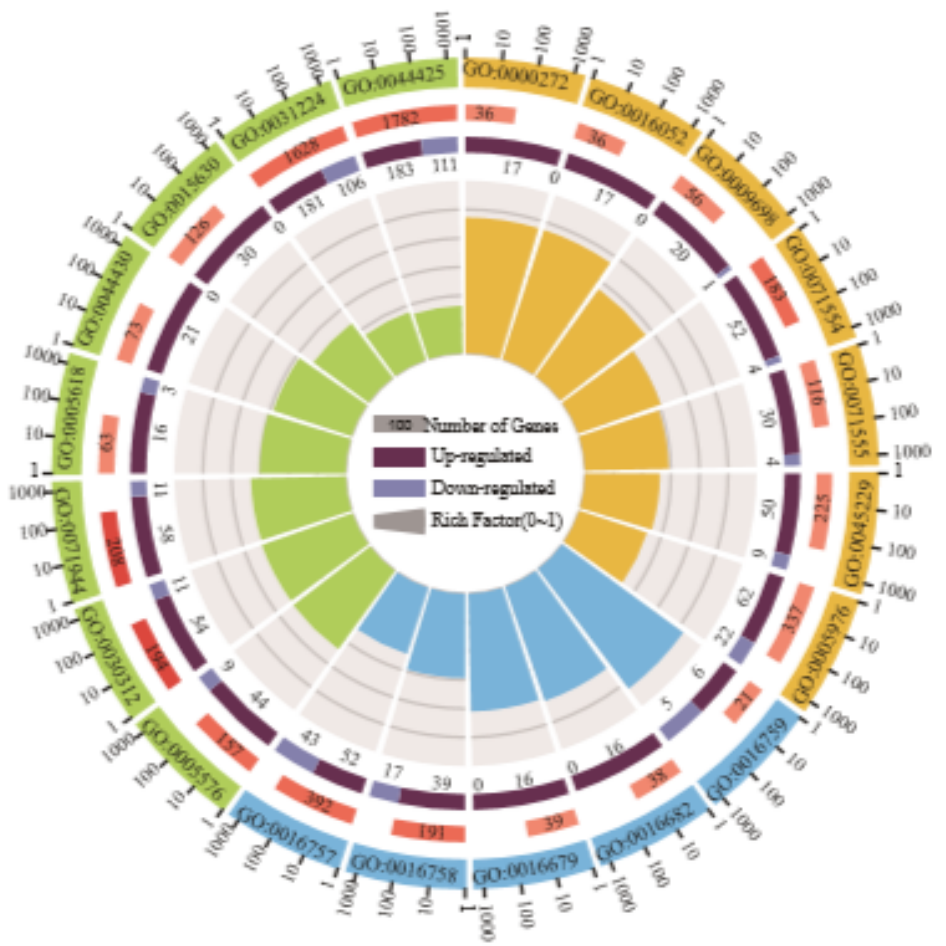

$$
\begin{aligned}
& \text { Biological Process } \\
& \text { Molecular Function } \\
& \text { Cellular Component } \\
& \\
& \text {-log10(Qvahue) } \\
& (0,1.3] \\
& (1.3,2] \\
& (2,5] \\
& (5,10] \\
& (10,15] \\
& (15,20] \\
& >=20
\end{aligned}
$$

B

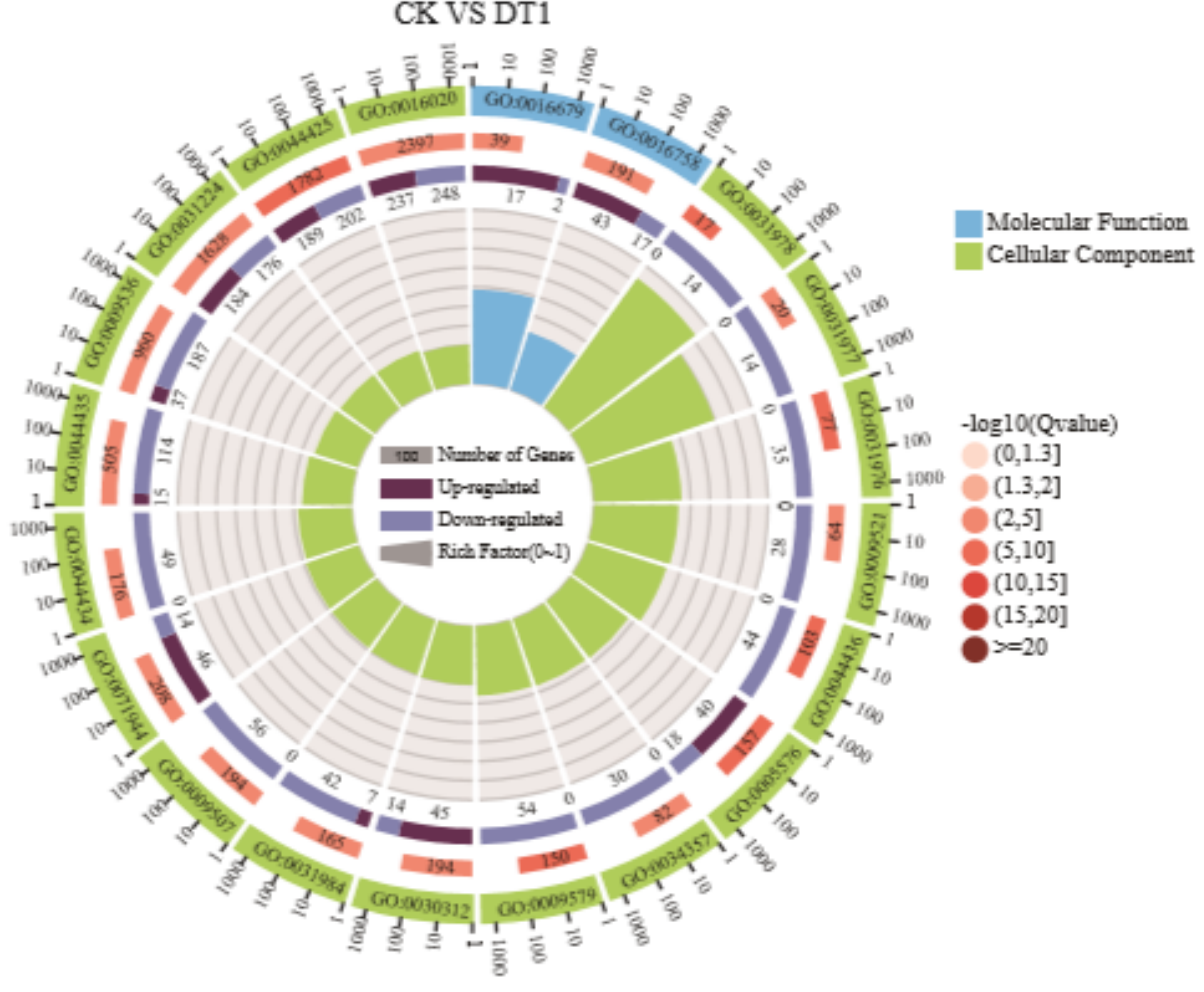

CK VS DT2

\section{Figure 4}

GO term analysis of control group and different treatment groups. (A) GO term analysis of CK vs DT1. (B) $\mathrm{GO}$ term analysis of CK vs DT2. The second lap represents the number of the genes in the genome 
background and the $Q$ values. The third lap represents the ratio of the upregulated genes (deep purple) and downregulated genes (light purple). The fourth lap represents the enrichment factor of each GO term.

A

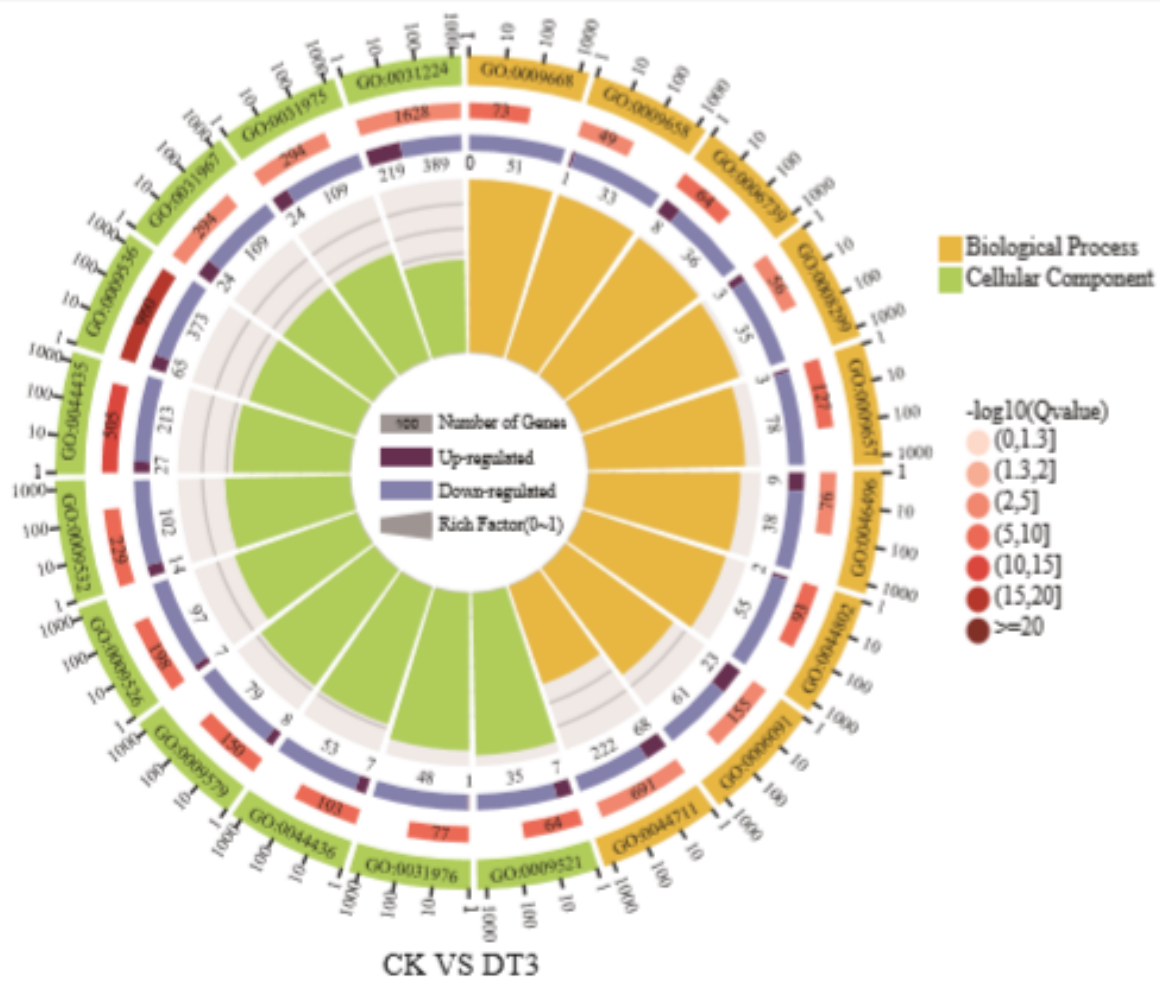

B

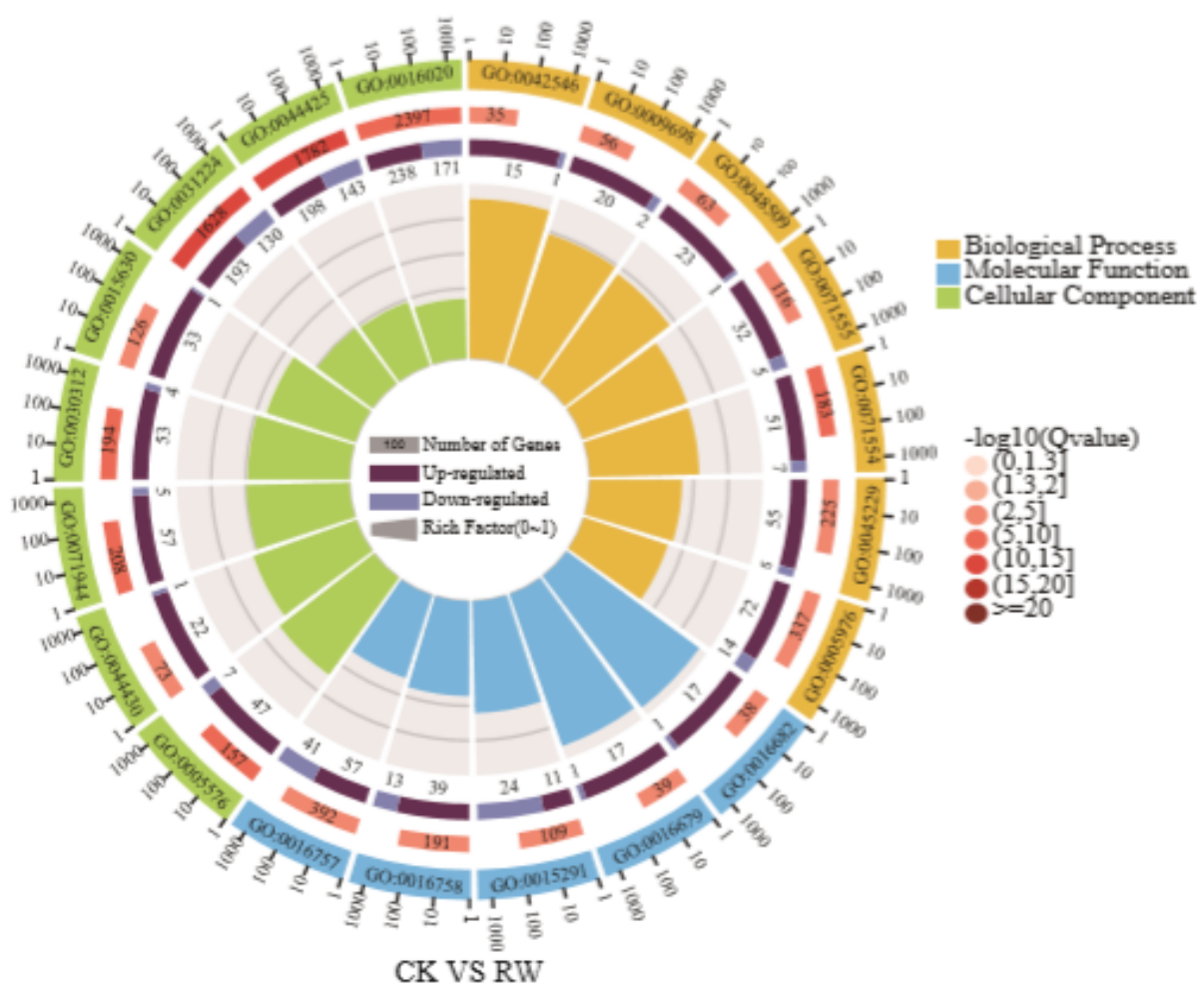

\section{Figure 5}

GO term analysis of control group and different treatment groups. (A) GO term analysis of CK vs DT3. (B) $\mathrm{GO}$ term analysis of CK vs RW. The first lap represents the top $20 \mathrm{GO}$ terms. The second lap represents the number of the genes in the genome background and the $Q$ values. The third lap represents the ratio of 
the upregulated genes (deep purple) and downregulated genes (light purple). The fourth lap represents the enrichment factor of each GO term.

A

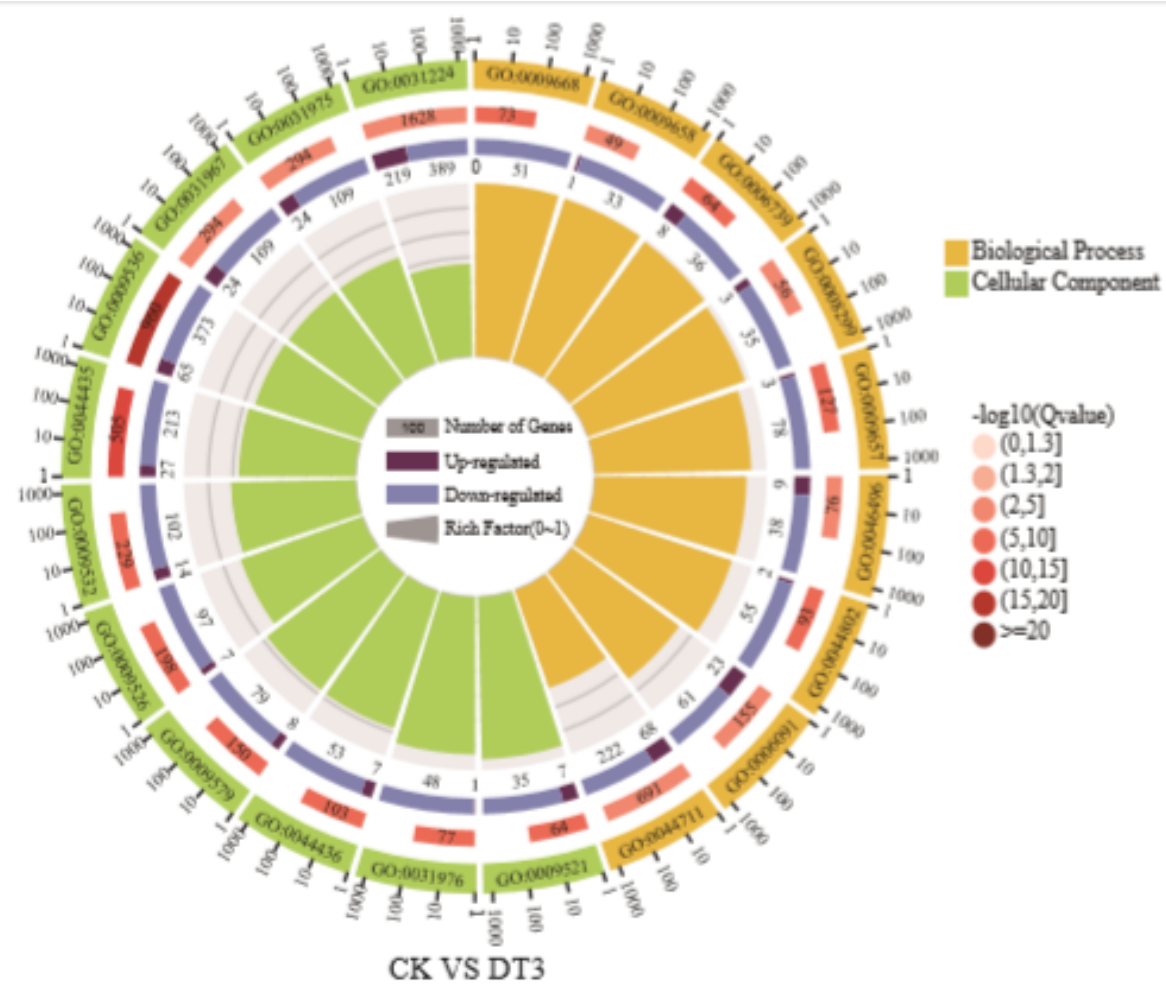

B

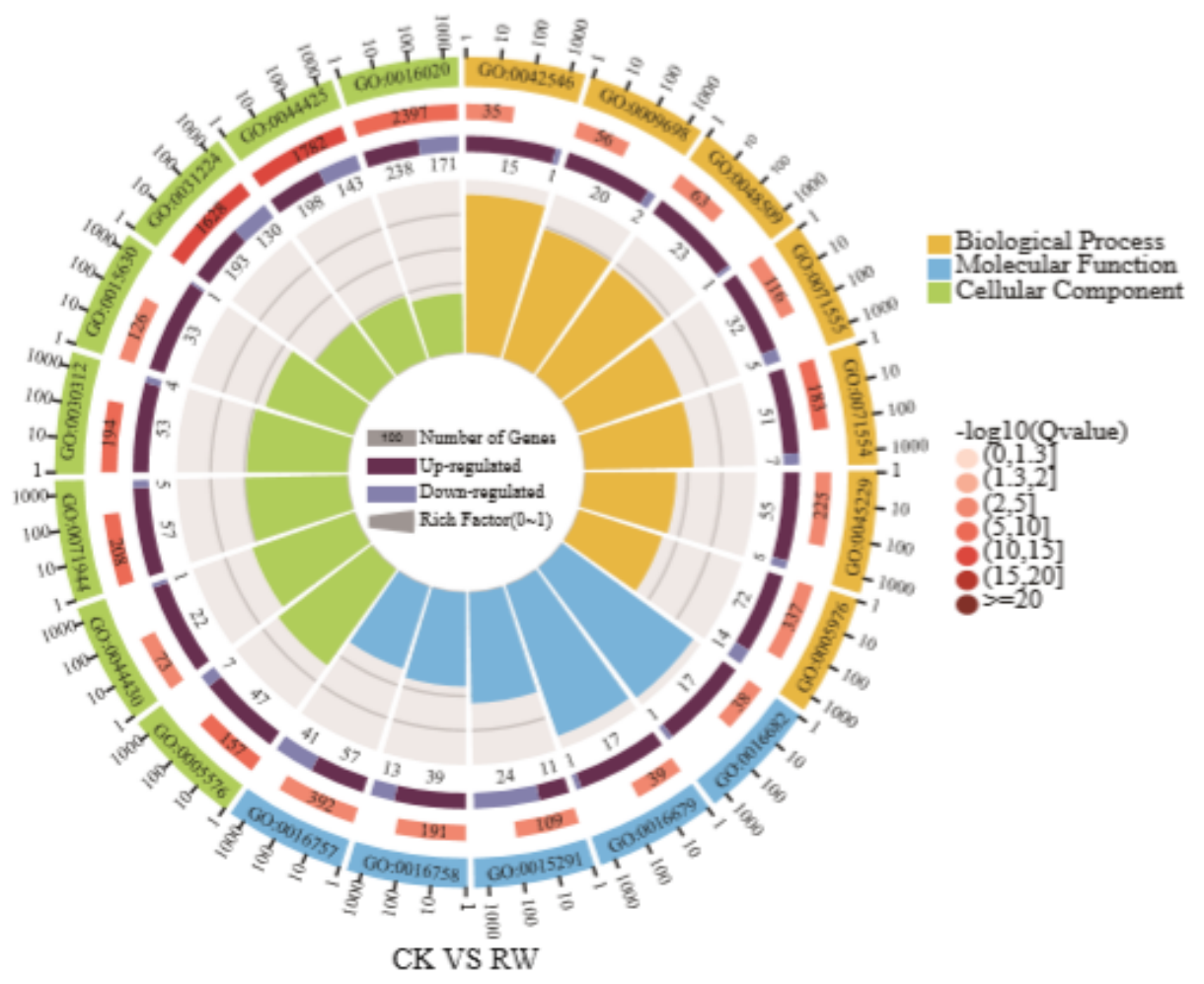

\section{Figure 5}

GO term analysis of control group and different treatment groups. (A) GO term analysis of CK vs DT3. (B) $\mathrm{GO}$ term analysis of CK vs RW. The first lap represents the top $20 \mathrm{GO}$ terms. The second lap represents the number of the genes in the genome background and the $Q$ values. The third lap represents the ratio of 
the upregulated genes (deep purple) and downregulated genes (light purple). The fourth lap represents the enrichment factor of each GO term.

A

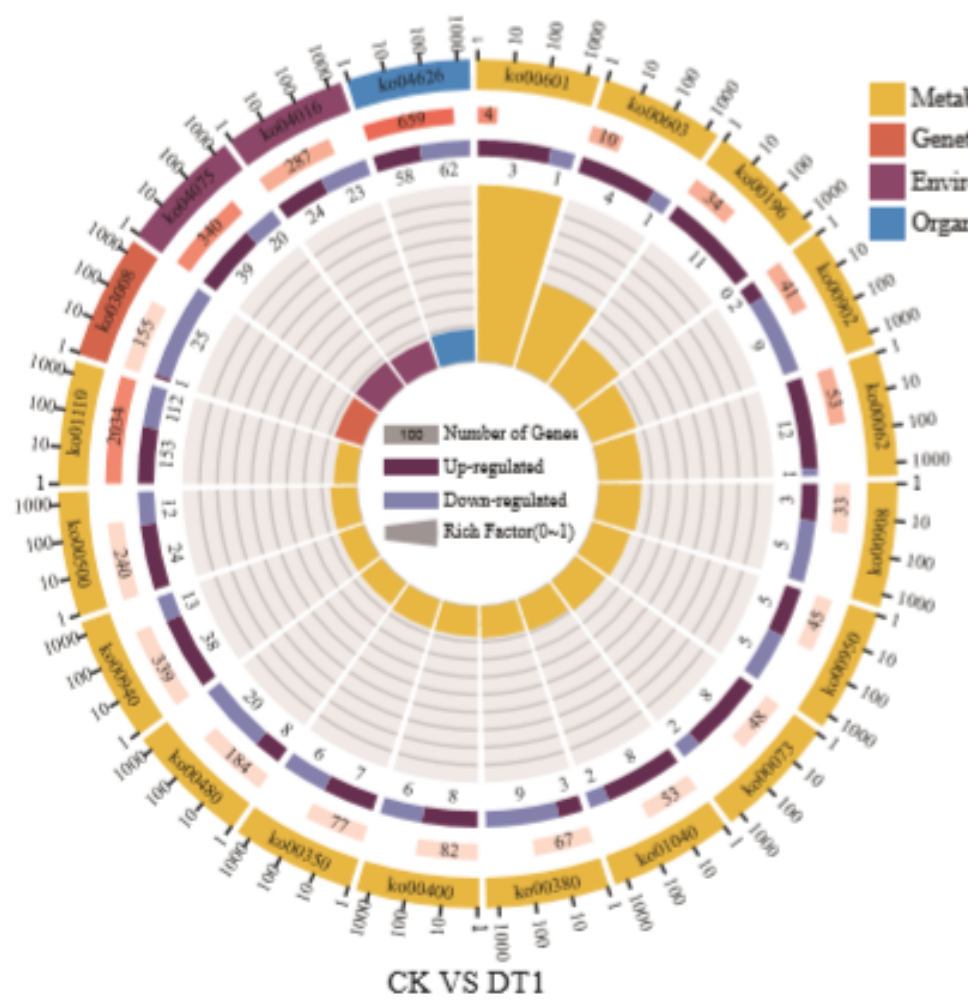

Metabolism

Genetic Information Processing

Emironmental Information Processing

Organismal Systems

-log 10 (Qvalue)

$(0,1.3]$

(1.3,2]

$(2,5]$

$(5,10]$

$(10,15]$

$(15,20]$

B

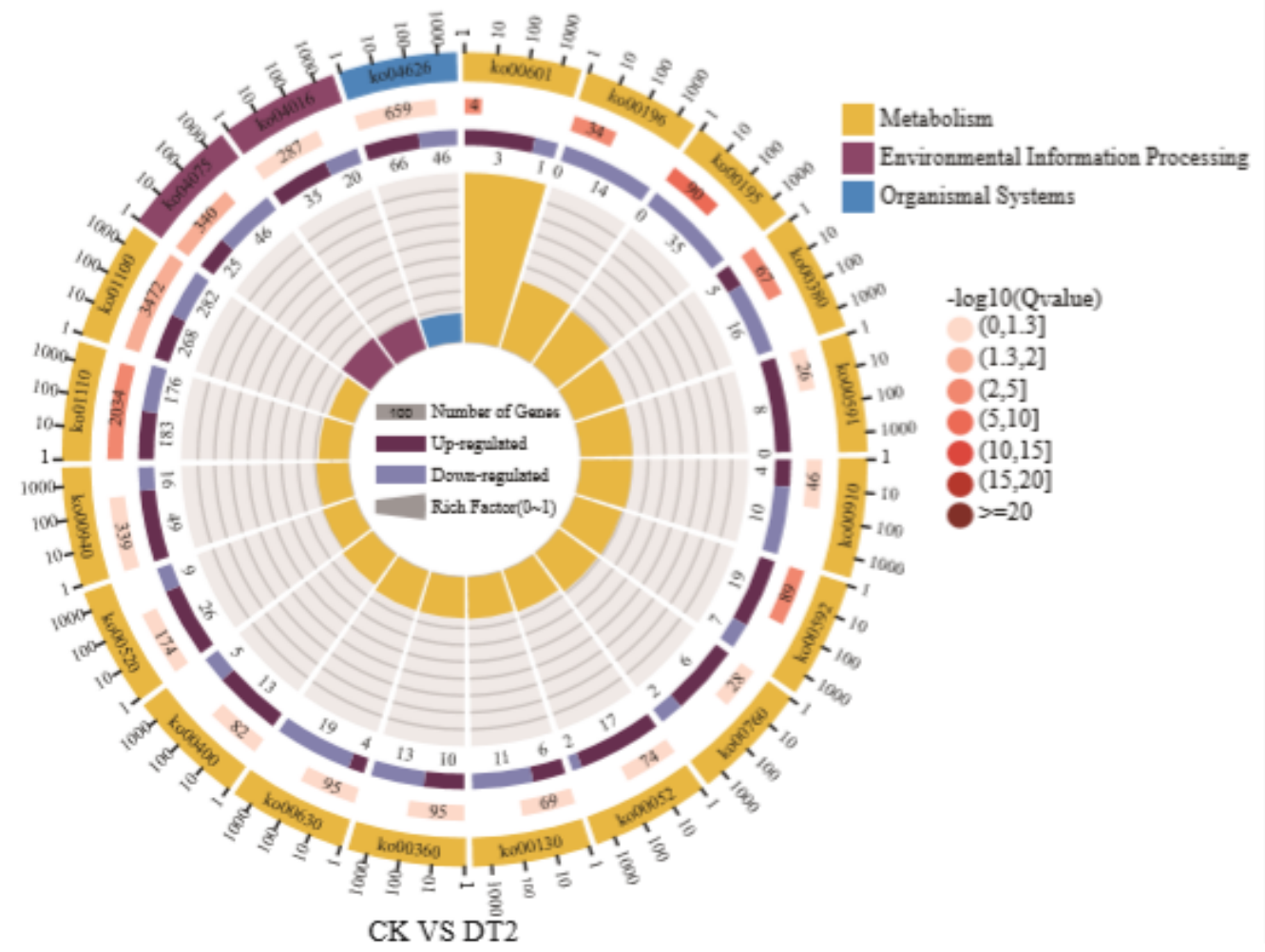

Figure 6

KEGG classification analysis of control group and different treatment groups. (A) KEGG classification analysis of CK vs DT1. (B) KEGG classification analysis of CK vs DT2. The second lap represents the number of the genes in the genome background and the $Q$ values. The third lap represents the ratio of the 
upregulated genes (deep purple) and downregulated genes (light purple). The fourth lap represents the enrichment factor of each pathway.

A

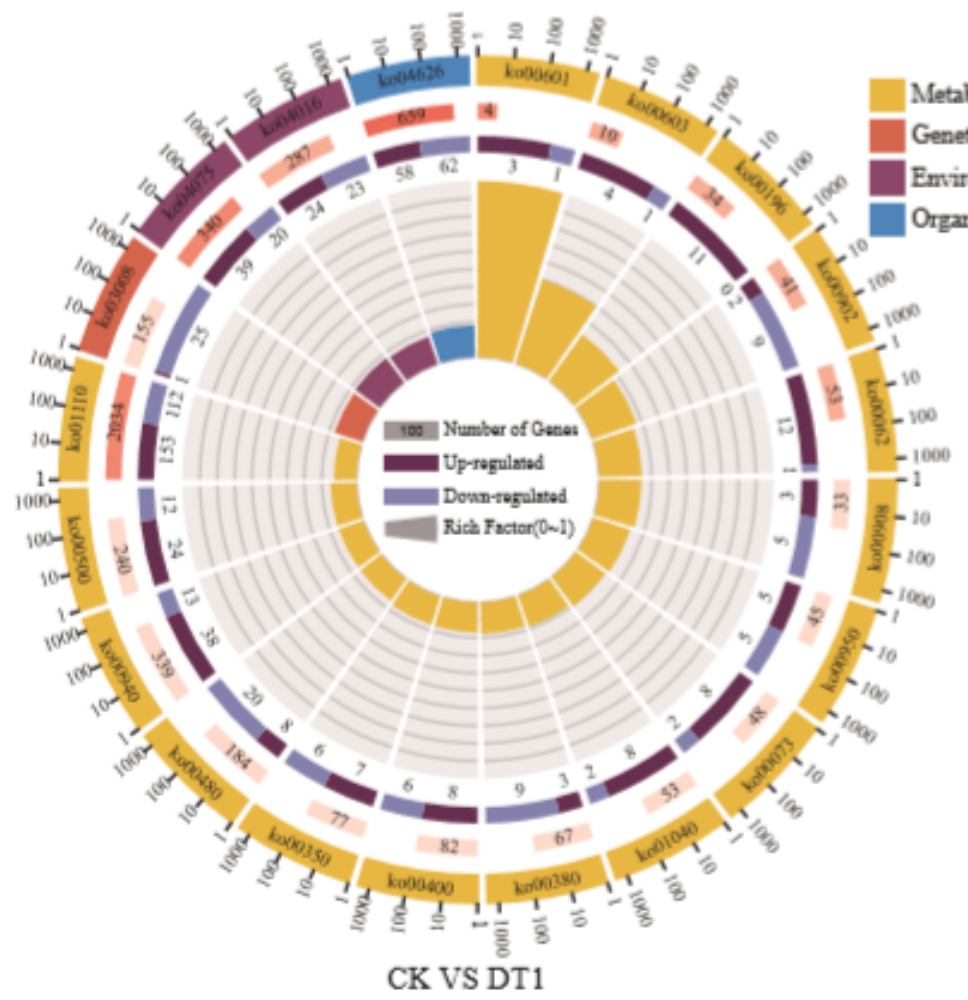

Metabolism

Genetic Information Processing

Emironmental Information Processing

Organismal Systems

-log 10 (Qvalue)

$(0,1.3]$

(1.3,2]

$(2,5]$

$(5,10]$

$(10,15]$

$(15,20]$

B

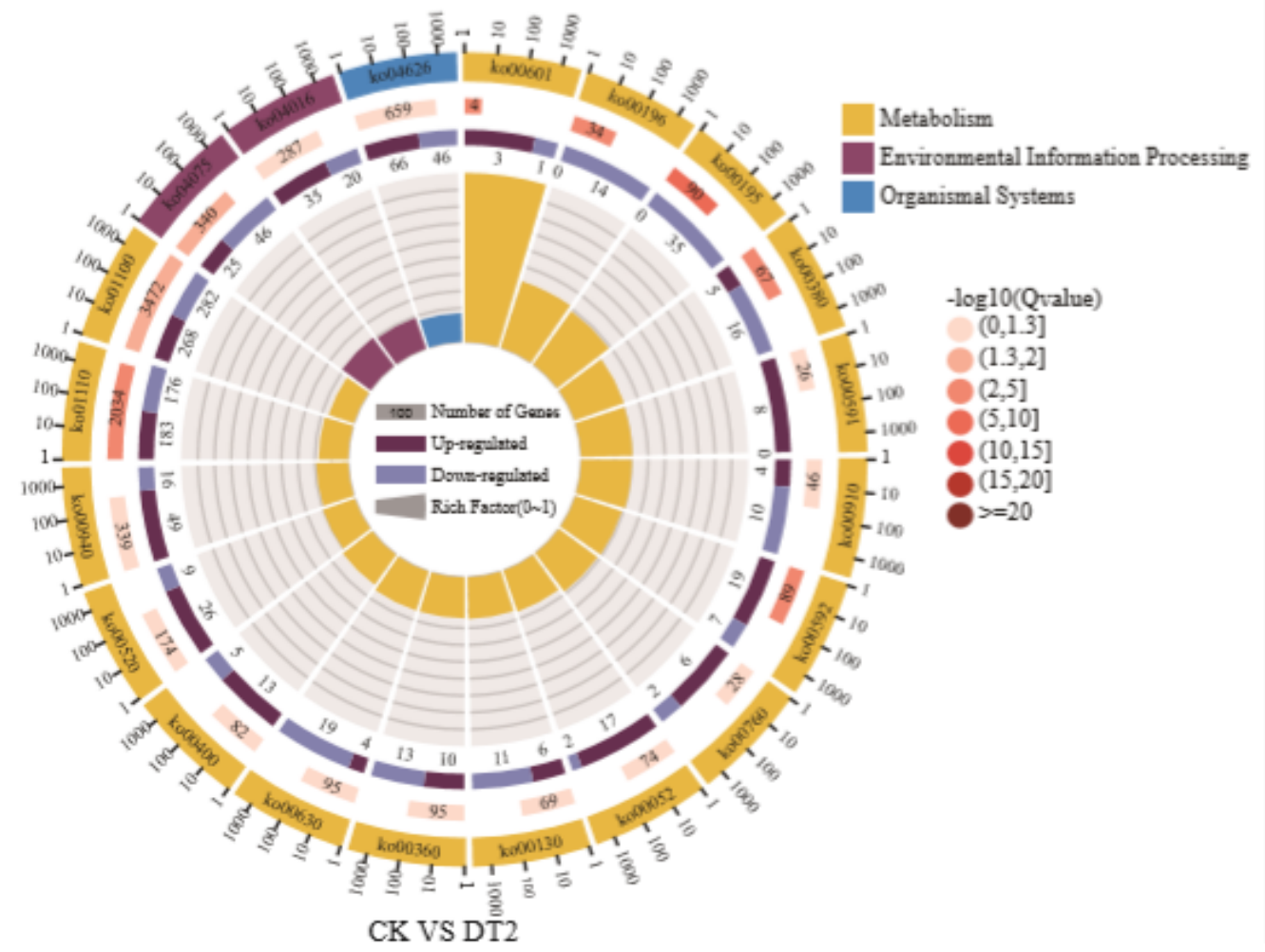

Figure 6

KEGG classification analysis of control group and different treatment groups. (A) KEGG classification analysis of CK vs DT1. (B) KEGG classification analysis of CK vs DT2. The second lap represents the number of the genes in the genome background and the $Q$ values. The third lap represents the ratio of the 
upregulated genes (deep purple) and downregulated genes (light purple). The fourth lap represents the enrichment factor of each pathway.

A

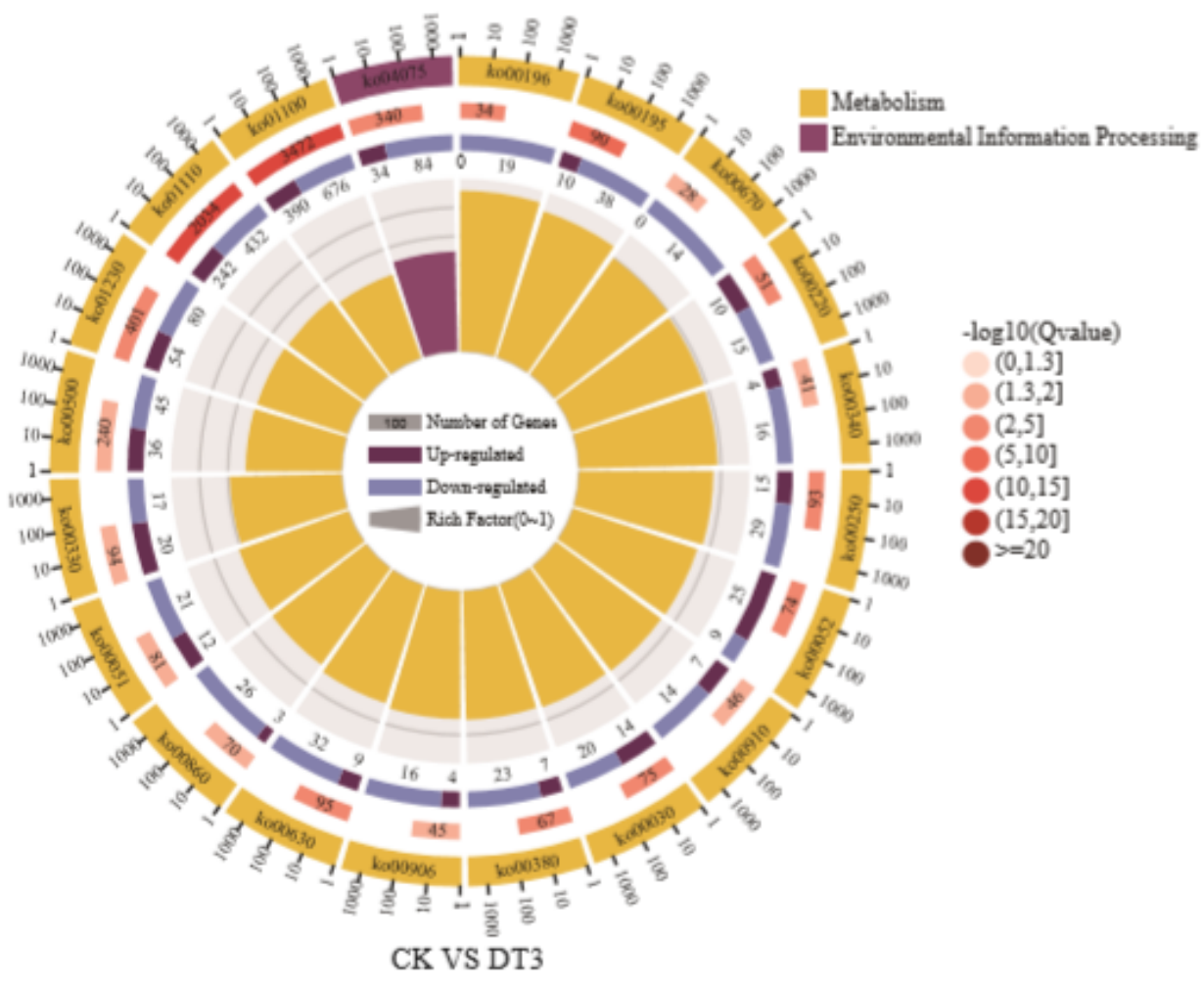

B

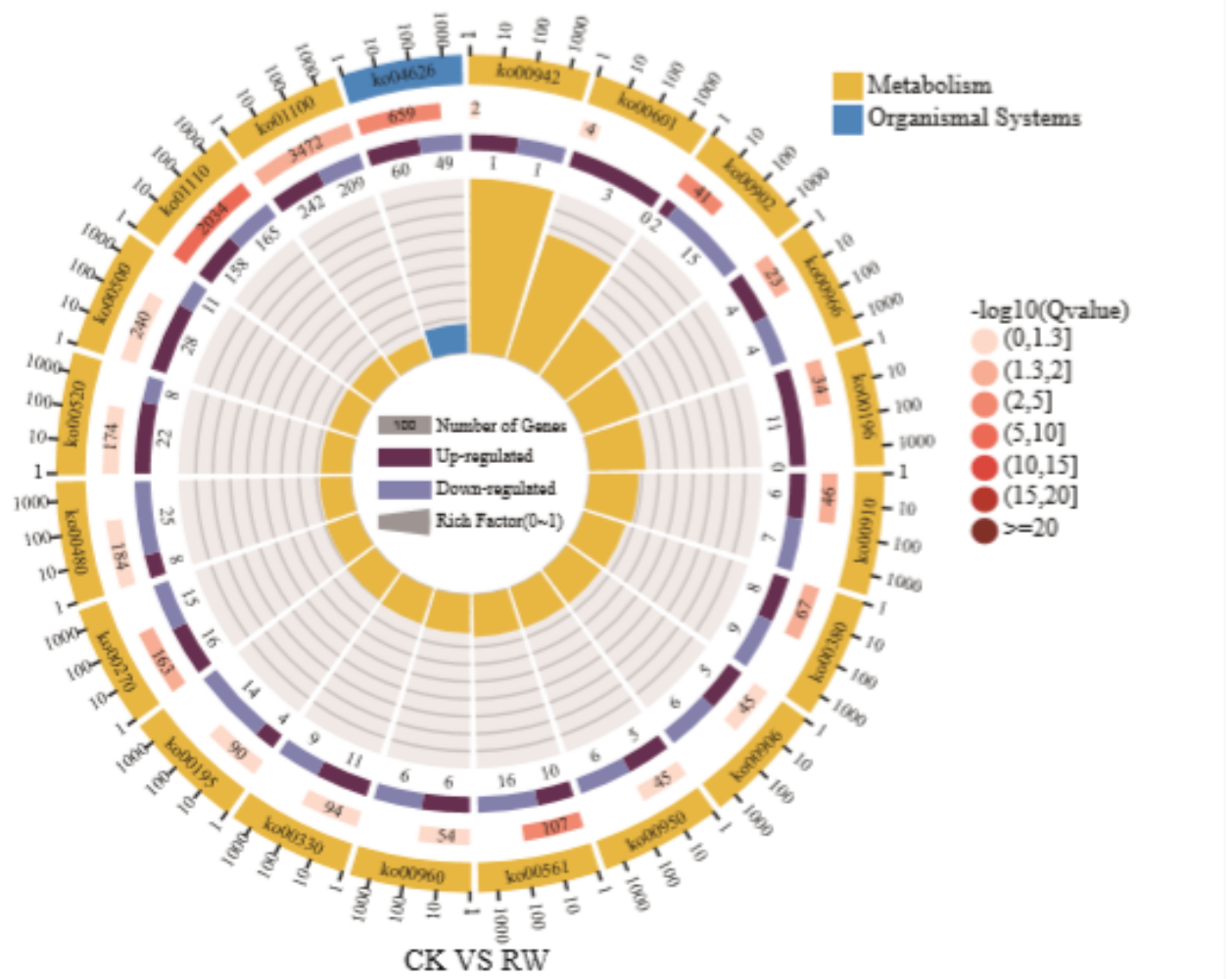

Figure 7

KEGG classification analysis of control group and different treatment groups. (A) KEGG classification analysis of CK vs DT3. (B) KEGG classification analysis of CK vs RW. The first lap represents the top 20 pathways in the enrichment. The second lap represents the number of the genes in the genome 
background and the $Q$ values. The third lap represents the ratio of the upregulated genes (deep purple) and downregulated genes (light purple). The fourth lap represents the enrichment factor of each pathway.

A

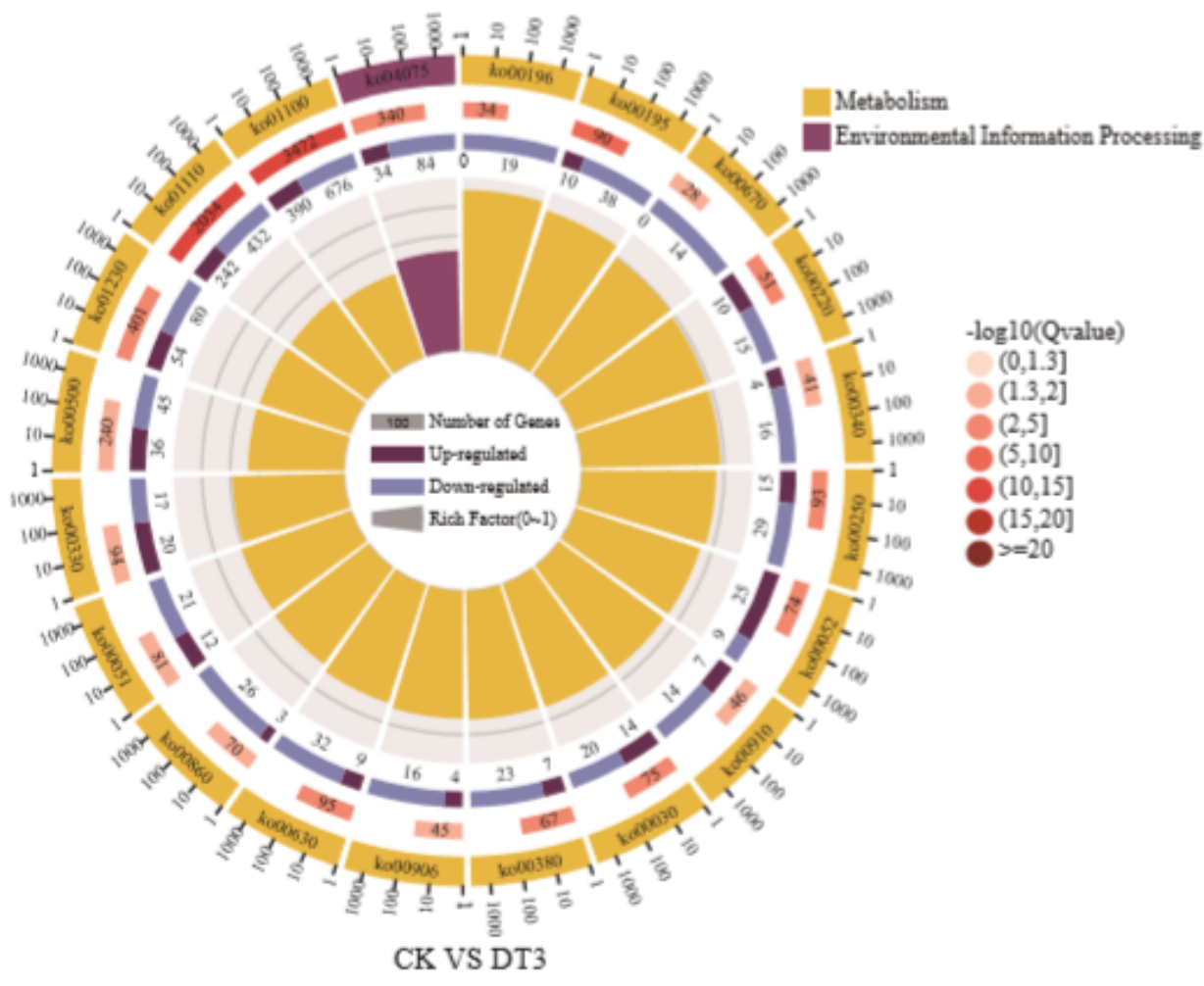

B

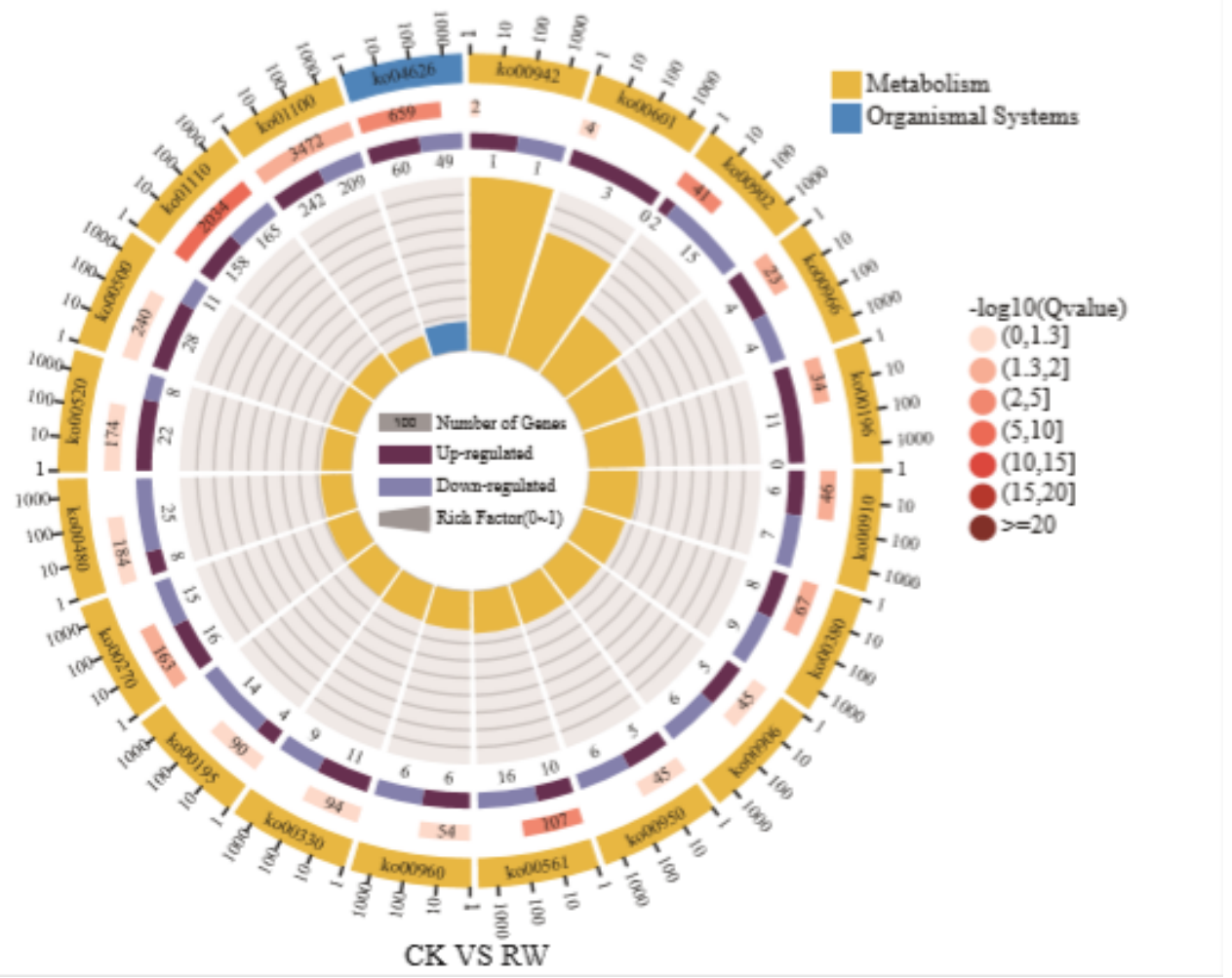

Figure 7

KEGG classification analysis of control group and different treatment groups. (A) KEGG classification analysis of CK vs DT3. (B) KEGG classification analysis of CK vs RW. The first lap represents the top 20 pathways in the enrichment. The second lap represents the number of the genes in the genome 
background and the $\mathrm{Q}$ values. The third lap represents the ratio of the upregulated genes (deep purple) and downregulated genes (light purple). The fourth lap represents the enrichment factor of each pathway.

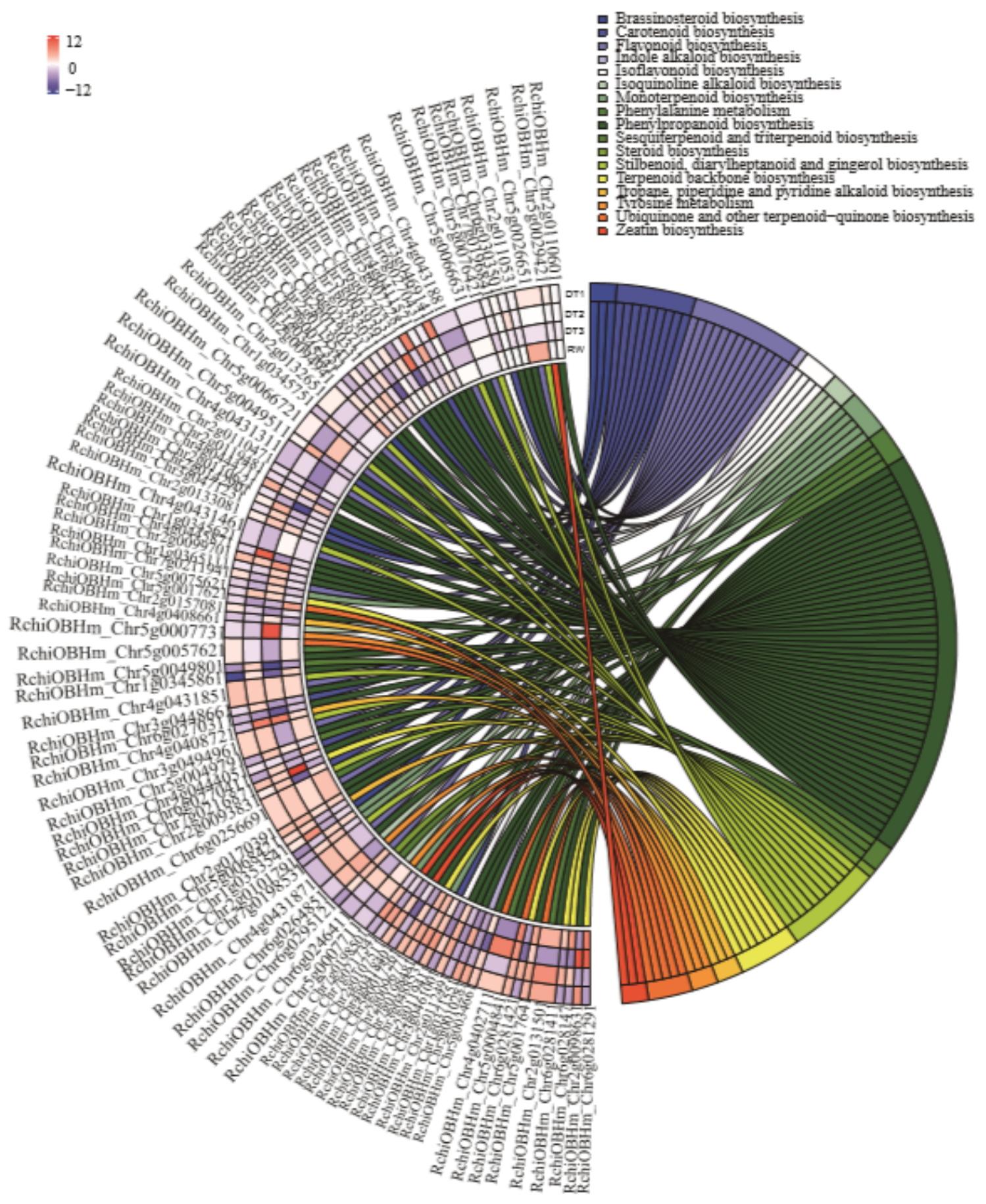

Figure 8

KEGG enrichment of differentially expressed genes involved in secondary metabolism. 


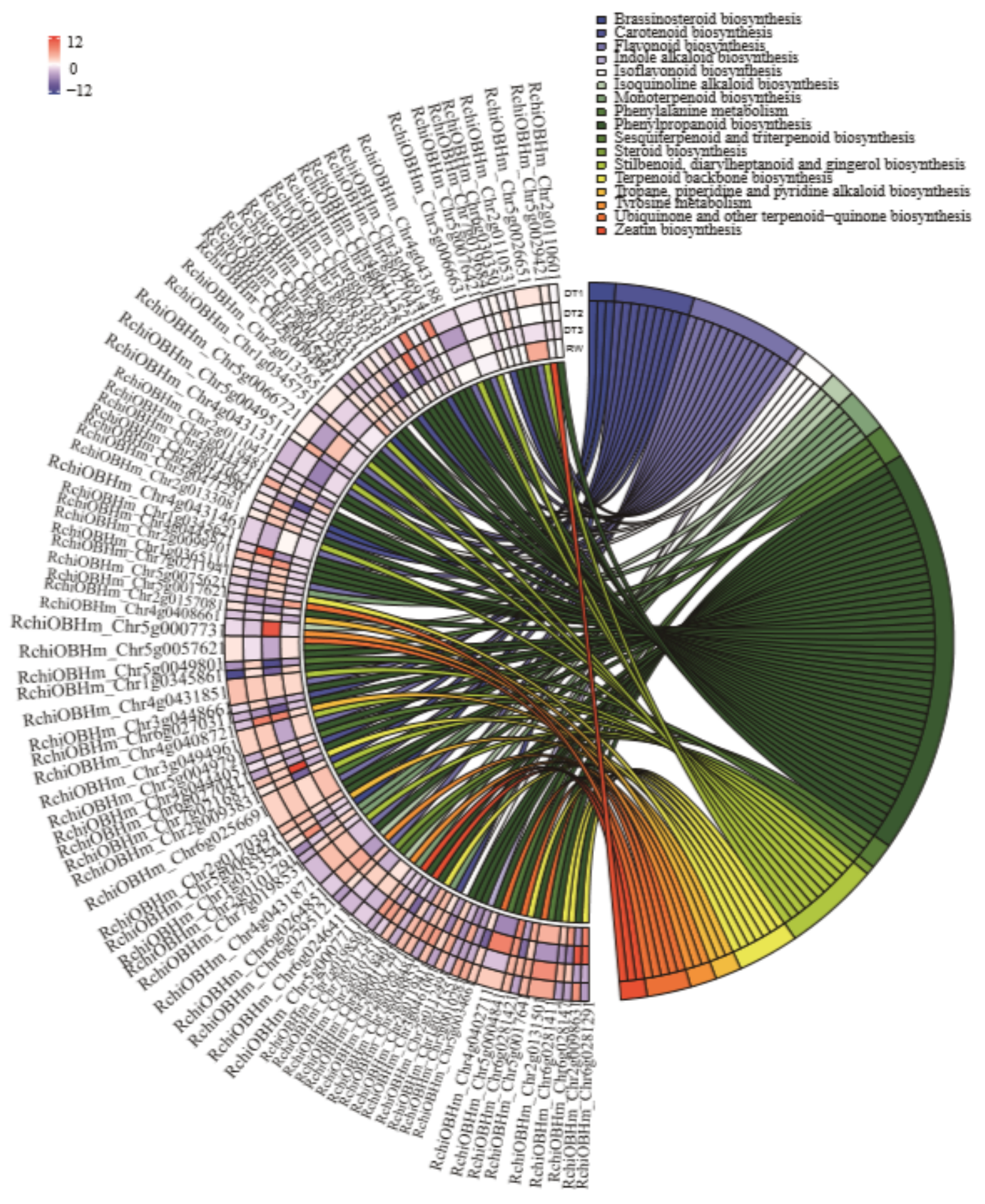

Figure 8

KEGG enrichment of differentially expressed genes involved in secondary metabolism. 


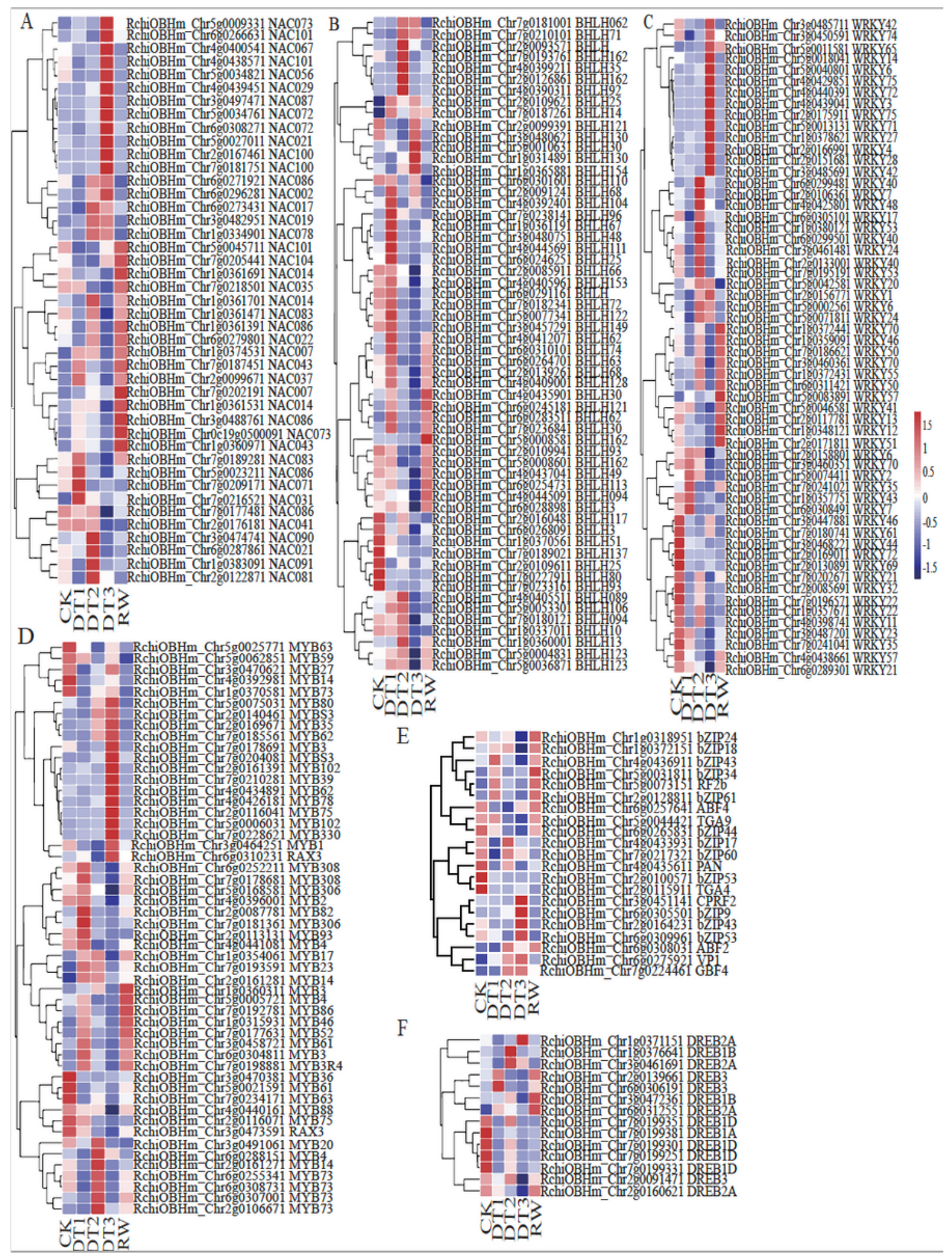

Figure 9

Heat map of different transcription factors in differentially expressed genes. (A) Heat map of NACs. (B) Heat map of bHLHs. (C) Heat map of WRKYs. (D) Heat map of MYBs. (E) Heat map of bZIPs. (F) Heat map of DREBs (The relative genes expression level come from DEGs data after taking log10(RPKM+1). Red indicates high expression level and blue indicates low expression level.) 


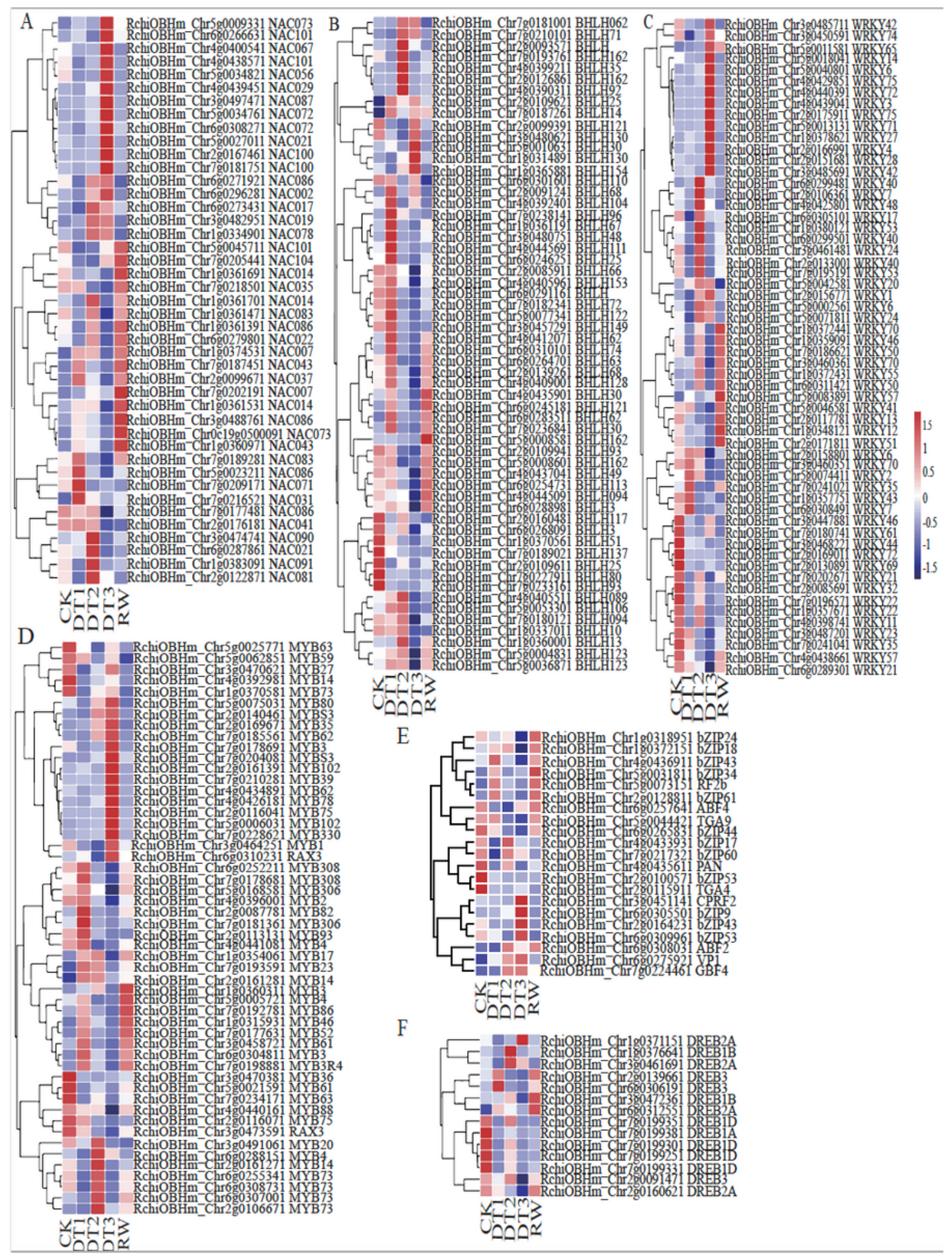

Figure 9

Heat map of different transcription factors in differentially expressed genes. (A) Heat map of NACs. (B) Heat map of bHLHs. (C) Heat map of WRKYs. (D) Heat map of MYBs. (E) Heat map of bZIPs. (F) Heat map of DREBs (The relative genes expression level come from DEGs data after taking log10(RPKM+1). Red indicates high expression level and blue indicates low expression level.) 

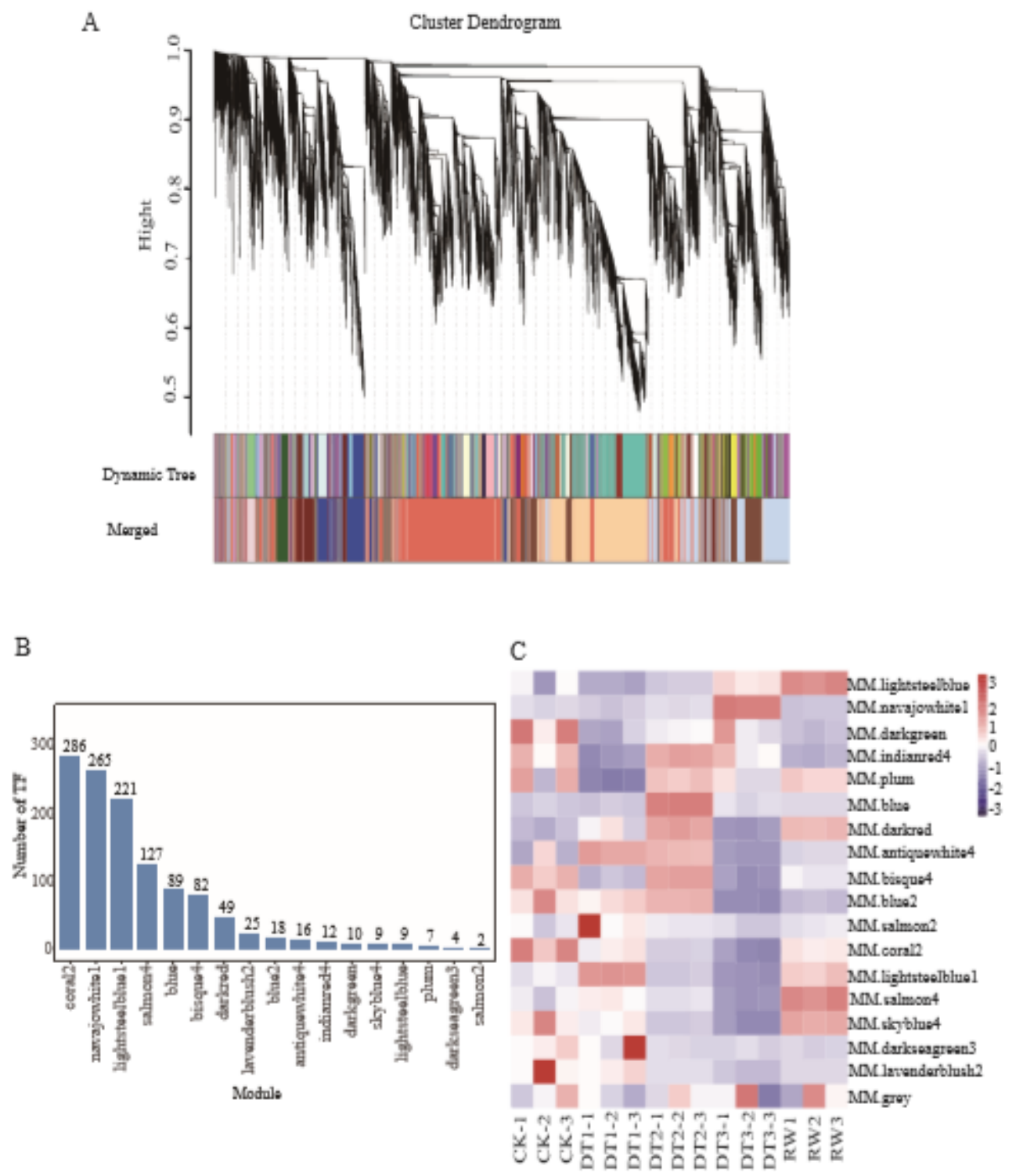

Figure 10

Identification of differentially expressed genes by WGCNA. (A) Module level clustering diagram. (B) TF numbers of each module. (C) Heat map of sample expression pattern. 

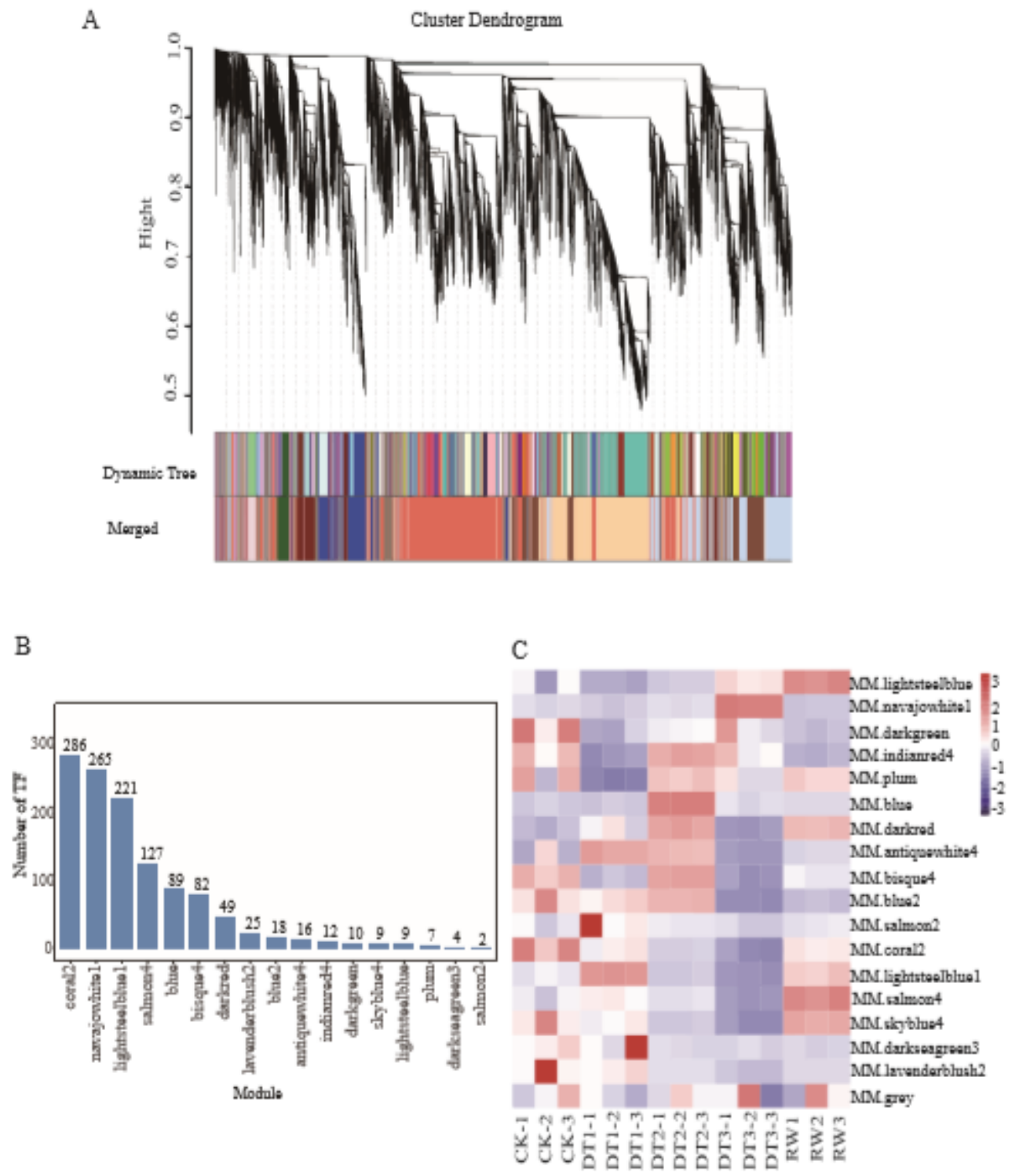

Figure 10

Identification of differentially expressed genes by WGCNA. (A) Module level clustering diagram. (B) TF numbers of each module. (C) Heat map of sample expression pattern. 
Module-Trait relationships

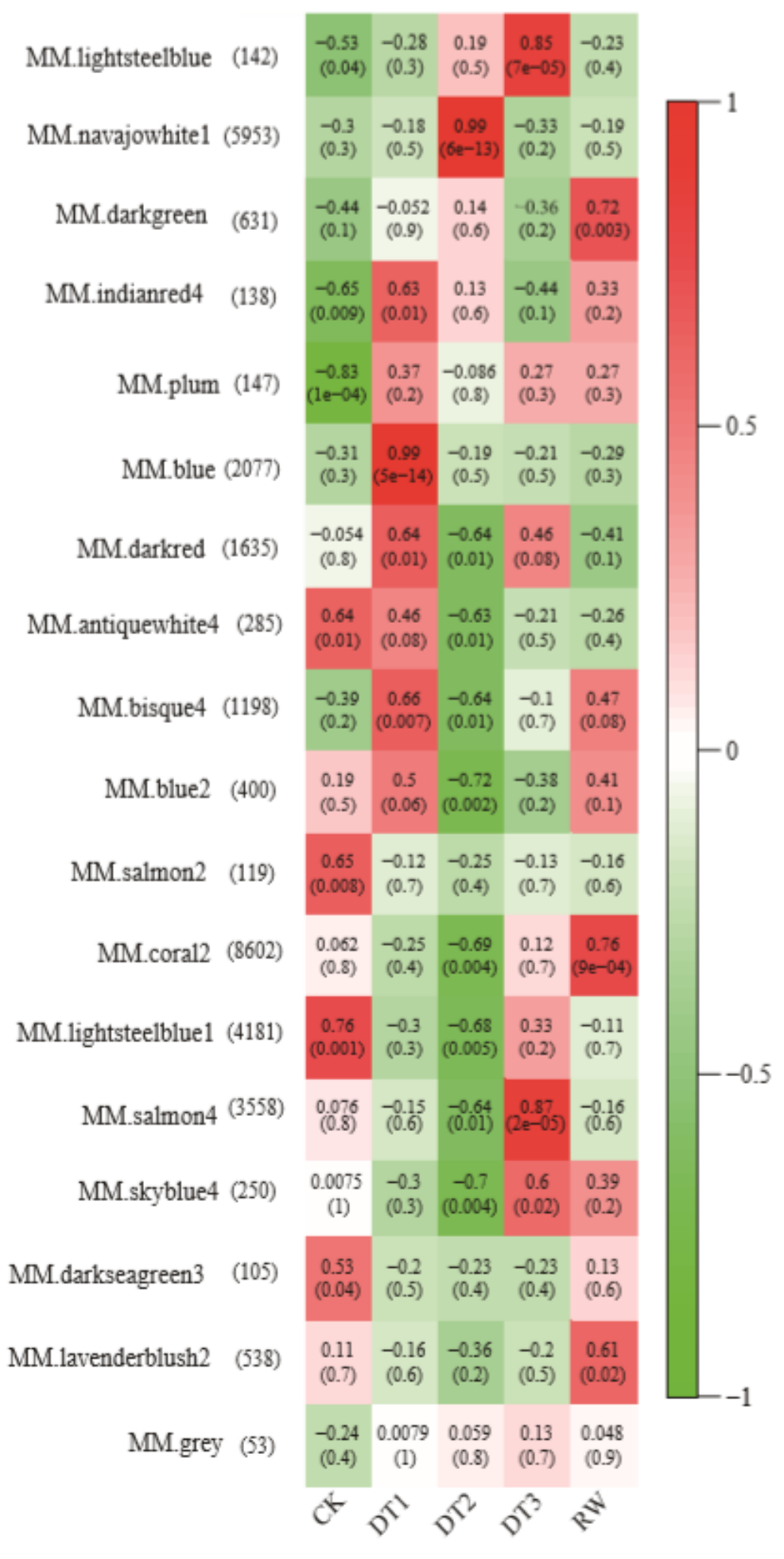

\section{Figure 11}

Module-trait associations using WGCNA. Each column corresponds to different processing conditions, and each row corresponds to the characteristic gene of the module. The correlation between the two is indicated in the cell by the Pearson correlation coefficient and the $\mathrm{p}$ value in parentheses. The cell color ranges from red (high positive correlation) to green (high negative correlation), and the number of genes contained in each module is in the left parentheses. 
Module-Trait relationships

\begin{tabular}{|c|c|c|c|c|c|c|c|}
\hline MM.lightsteelblue & $(142)$ & $\begin{array}{l}-0.53 \\
(0.04)\end{array}$ & $\begin{array}{c}-0.28 \\
(0.3)\end{array}$ & $\begin{array}{l}0.19 \\
(0.5)\end{array}$ & $\begin{array}{c}0.85 \\
(7 e-0.5)\end{array}$ & $\begin{array}{c}-0.23 \\
(0.4)\end{array}$ & \\
\hline MM.navajowhite1 & (5953) & $\begin{array}{l}-0.3 \\
(0.3)\end{array}$ & $\begin{array}{c}-0.18 \\
(0.5)\end{array}$ & $\begin{array}{c}0.99 \\
(6 e-13)\end{array}$ & $\begin{array}{l}-0.33 \\
(0.2)\end{array}$ & $\begin{array}{c}-0.19 \\
(0.5)\end{array}$ & \\
\hline MM.darkgreen & $(631)$ & $\begin{array}{c}-0.44 \\
(0.1)\end{array}$ & $\begin{array}{c}-0.052 \\
(0.9)\end{array}$ & $\begin{array}{l}0.14 \\
(0.6)\end{array}$ & $\begin{array}{l}-0.36 \\
(0.2)\end{array}$ & $\begin{array}{c}0.72 \\
(0.003)\end{array}$ & \\
\hline MM.indianred4 & (138) & $\begin{array}{l}-0.65 \\
(0.009)\end{array}$ & $\begin{array}{c}0.63 \\
(0.01)\end{array}$ & $\begin{array}{l}0.13 \\
(0.6)\end{array}$ & $\begin{array}{c}-0.44 \\
(0.1)\end{array}$ & $\begin{array}{l}0.33 \\
(0.2)\end{array}$ & \\
\hline MM.plum & $(147)$ & $\begin{array}{c}-0.83 \\
(1 \mathrm{e}-04)\end{array}$ & $\begin{array}{l}0.37 \\
(0.2)\end{array}$ & $\begin{array}{c}-0.086 \\
(0.8)\end{array}$ & $\begin{array}{l}0.27 \\
(0.3)\end{array}$ & $\begin{array}{l}0.27 \\
(0.3)\end{array}$ & \\
\hline MM.blue & (2077) & $\begin{array}{c}-0.31 \\
(0.3)\end{array}$ & $\begin{array}{c}0.99 \\
(5 e-14)\end{array}$ & $\begin{array}{c}-0.19 \\
(0.5)\end{array}$ & $\begin{array}{c}-0.21 \\
(0.5)\end{array}$ & $\begin{array}{r}-0.29 \\
(0.3)\end{array}$ & \\
\hline MM.darkred & (1635) & $\begin{array}{c}-0.054 \\
(0.8)\end{array}$ & $\begin{array}{c}0.64 \\
(0.01)\end{array}$ & $\begin{array}{c}-0.64 \\
(0.01)\end{array}$ & $\begin{array}{c}0.46 \\
(0.08)\end{array}$ & $\begin{array}{c}-0.41 \\
(0.1)\end{array}$ & \\
\hline MM.antiquewhite4 & (285) & $\begin{array}{c}0.64 \\
(0.01)\end{array}$ & $\begin{array}{c}0.46 \\
(0.08)\end{array}$ & $\begin{array}{l}-0.63 \\
(0.01)\end{array}$ & $\begin{array}{l}-0.21 \\
(0.5)\end{array}$ & $\begin{array}{c}-0.26 \\
(0.4)\end{array}$ & \\
\hline MM.bisque4 & (1198) & $\begin{array}{c}-0.39 \\
(0.2)\end{array}$ & $\begin{array}{c}0.66 \\
(0.007)\end{array}$ & $\begin{array}{l}-0.64 \\
(0.01)\end{array}$ & $\begin{array}{l}-0.1 \\
(0.7)\end{array}$ & $\begin{array}{l}0.47 \\
(0.08)\end{array}$ & \\
\hline MM.blue2 & $(400)$ & $\begin{array}{l}0.19 \\
(0.5)\end{array}$ & $\begin{array}{c}0.5 \\
(0.06)\end{array}$ & $\begin{array}{c}-0.72 \\
(0.002)\end{array}$ & $\begin{array}{c}-0.38 \\
(0.2)\end{array}$ & $\begin{array}{l}0.41 \\
(0.1)\end{array}$ & \\
\hline MM.salmon2 & (119) & $\begin{array}{c}0.65 \\
(0.008)\end{array}$ & $\begin{array}{c}-0.12 \\
(0.7)\end{array}$ & $\begin{array}{c}-0.25 \\
(0.4)\end{array}$ & $\begin{array}{c}-0.13 \\
(0.7)\end{array}$ & $\begin{array}{c}-0.16 \\
(0.6)\end{array}$ & \\
\hline MM.coral2 & $(8602)$ & $\begin{array}{l}0.062 \\
(0.8)\end{array}$ & $\begin{array}{l}-0.25 \\
(0.4)\end{array}$ & $\begin{array}{l}-0.69 \\
(0.004)\end{array}$ & $\begin{array}{l}0.12 \\
(0.7)\end{array}$ & $\begin{array}{c}0.76 \\
(9 e-04)\end{array}$ & \\
\hline MM.lightsteelblue1 & (4181) & $\begin{array}{c}0.76 \\
(0.001)\end{array}$ & $\begin{array}{l}-0.3 \\
(0.3)\end{array}$ & $\begin{array}{l}-0.68 \\
(0.005)\end{array}$ & $\begin{array}{l}0.33 \\
(0.2)\end{array}$ & $\begin{array}{c}-0.11 \\
(0.7)\end{array}$ & \\
\hline MM.salmon4 & (3558) & $\begin{array}{l}0.076 \\
(0.8)\end{array}$ & $\begin{array}{l}-0.15 \\
(0.6)\end{array}$ & $\frac{-0.64}{(0.01)}$ & $(2 e-05)$ & $\begin{array}{l}-0.16 \\
(0.6)\end{array}$ & \\
\hline MM.skyblue4 & $(250)$ & $\begin{array}{c}0.0075 \\
\text { (1) }\end{array}$ & $\begin{array}{l}-0.3 \\
(0.3)\end{array}$ & $\begin{array}{c}-0.7 \\
(0.004)\end{array}$ & $\begin{array}{c}0.6 \\
(0.02)\end{array}$ & $\begin{array}{l}0.39 \\
(0.2)\end{array}$ & \\
\hline MM.darkseagreen 3 & $(105)$ & $\begin{array}{c}0.53 \\
(0.04)\end{array}$ & $\begin{array}{l}-0.2 \\
(0.5)\end{array}$ & $\begin{array}{c}-0.23 \\
(0.4)\end{array}$ & $\begin{array}{c}-0.23 \\
(0.4)\end{array}$ & $\begin{array}{l}0.13 \\
(0.6)\end{array}$ & \\
\hline MM.lavenderblush2 & (538) & $\begin{array}{l}0.11 \\
(0.7)\end{array}$ & $\begin{array}{c}-0.16 \\
(0.6)\end{array}$ & $\begin{array}{c}-0.36 \\
(0.2)\end{array}$ & $\begin{array}{l}-0.2 \\
(0.5)\end{array}$ & $\begin{array}{c}0.61 \\
(0.02)\end{array}$ & \\
\hline MM.grey & $(53)$ & $\begin{array}{c}-0.24 \\
(0.4)\end{array}$ & $\begin{array}{c}0.0079 \\
\text { (1) }\end{array}$ & $\begin{array}{l}0.059 \\
(0.8)\end{array}$ & $\begin{array}{l}0.13 \\
(0.7)\end{array}$ & $\begin{array}{c}0.048 \\
(0.9)\end{array}$ & \\
\hline
\end{tabular}

\section{Figure 11}

Module-trait associations using WGCNA. Each column corresponds to different processing conditions, and each row corresponds to the characteristic gene of the module. The correlation between the two is indicated in the cell by the Pearson correlation coefficient and the $\mathrm{p}$ value in parentheses. The cell color ranges from red (high positive correlation) to green (high negative correlation), and the number of genes contained in each module is in the left parentheses. 
A

$\mathrm{C}$

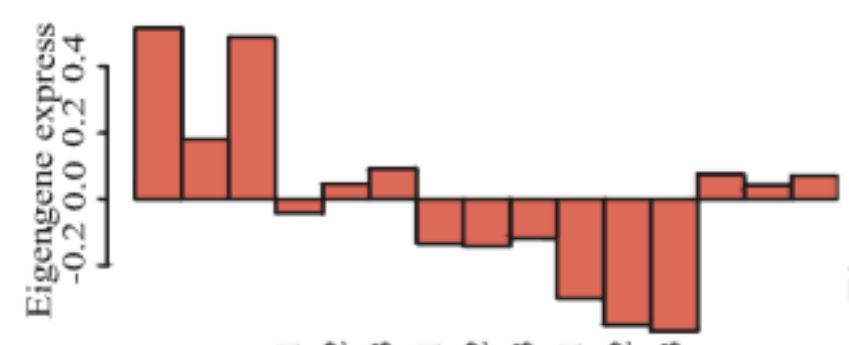

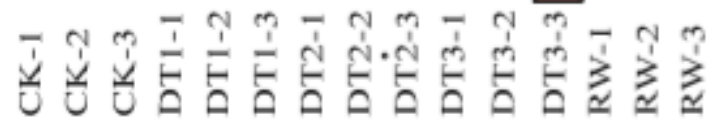
coral 2

B

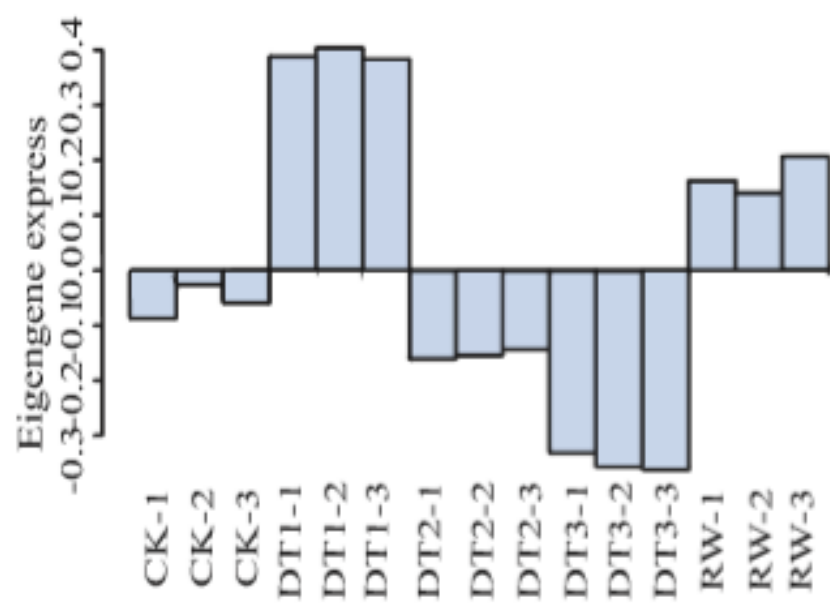

lightsteelblue 1

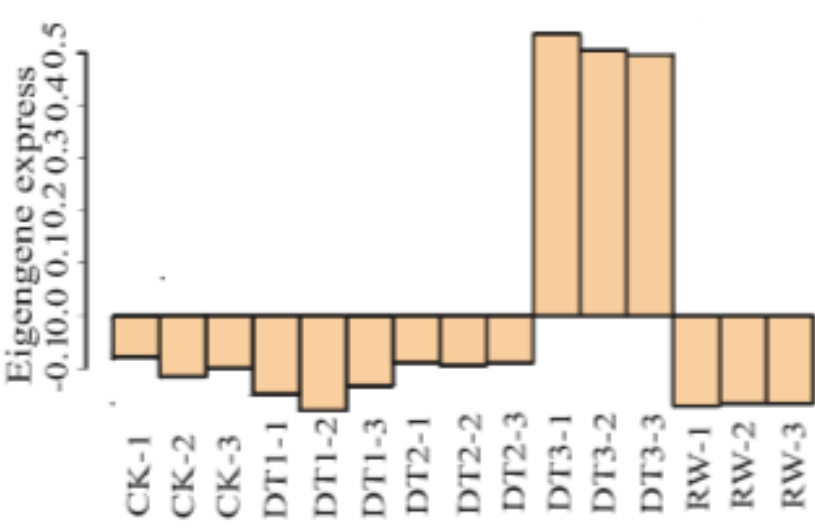

navajowhite 1

D

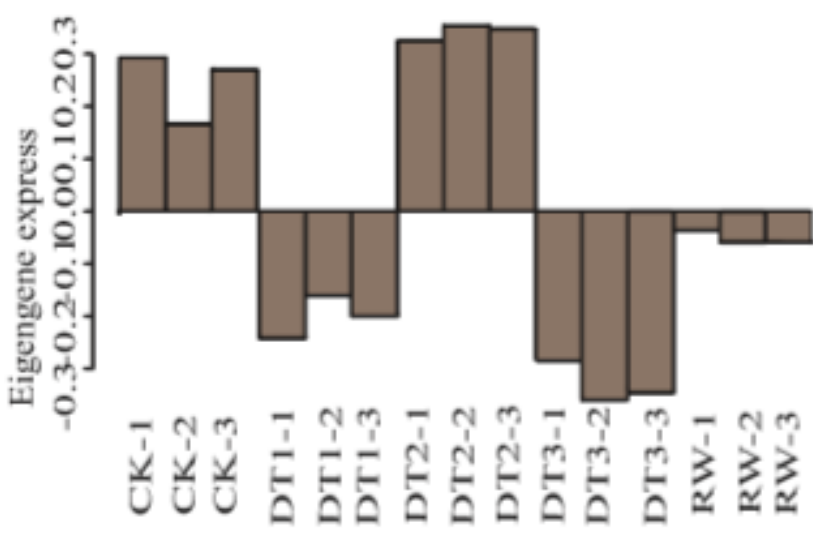

bisque 4

\section{Figure 12}

The expression pattern of the co-expressed genes in the representative module. (A) coral2 module. (B) lightsteelblue1 module. (C) navajowhite1 module. (D) bisque4 module. 
A

$\mathrm{C}$

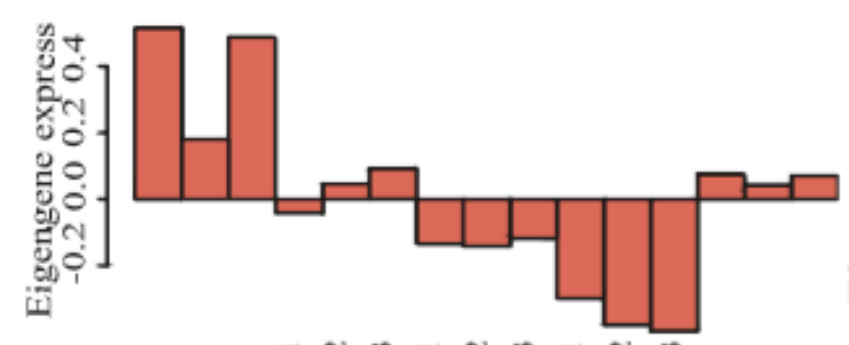

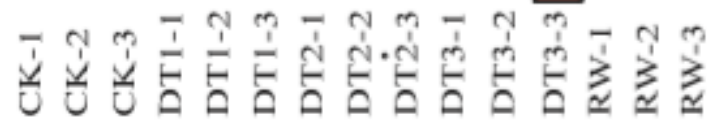
coral 2

B

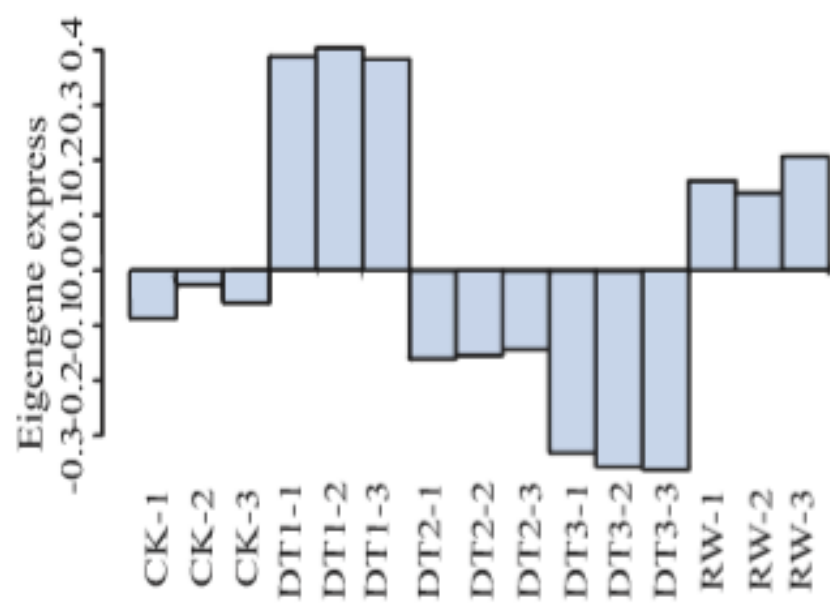

lightsteelblue 1

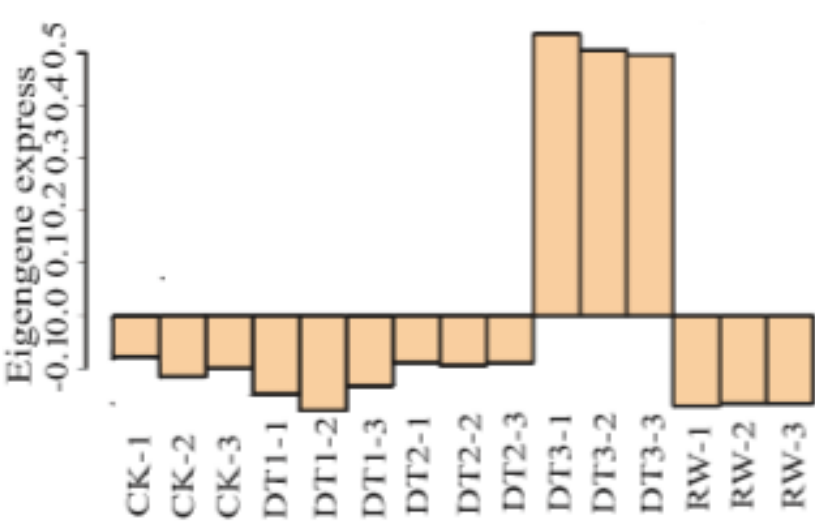

navajowhite 1

D

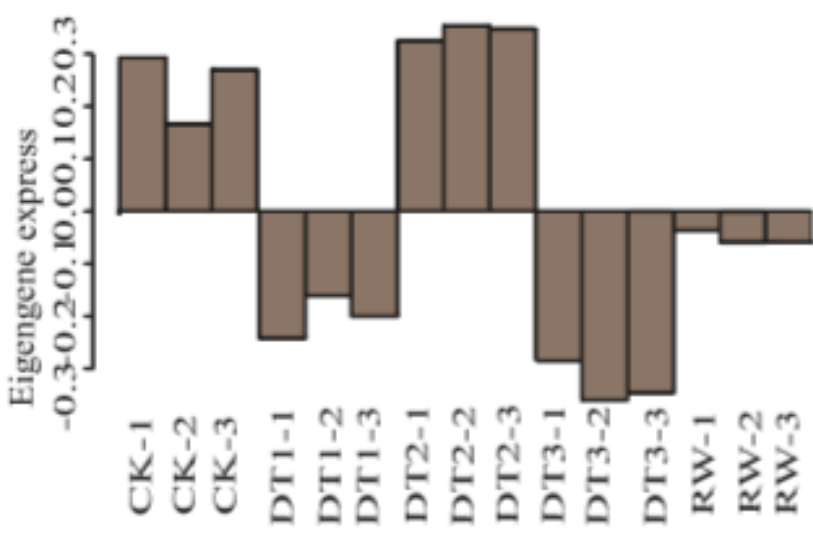

bisque 4

\section{Figure 12}

The expression pattern of the co-expressed genes in the representative module. (A) coral2 module. (B) lightsteelblue1 module. (C) navajowhite1 module. (D) bisque4 module. 
A

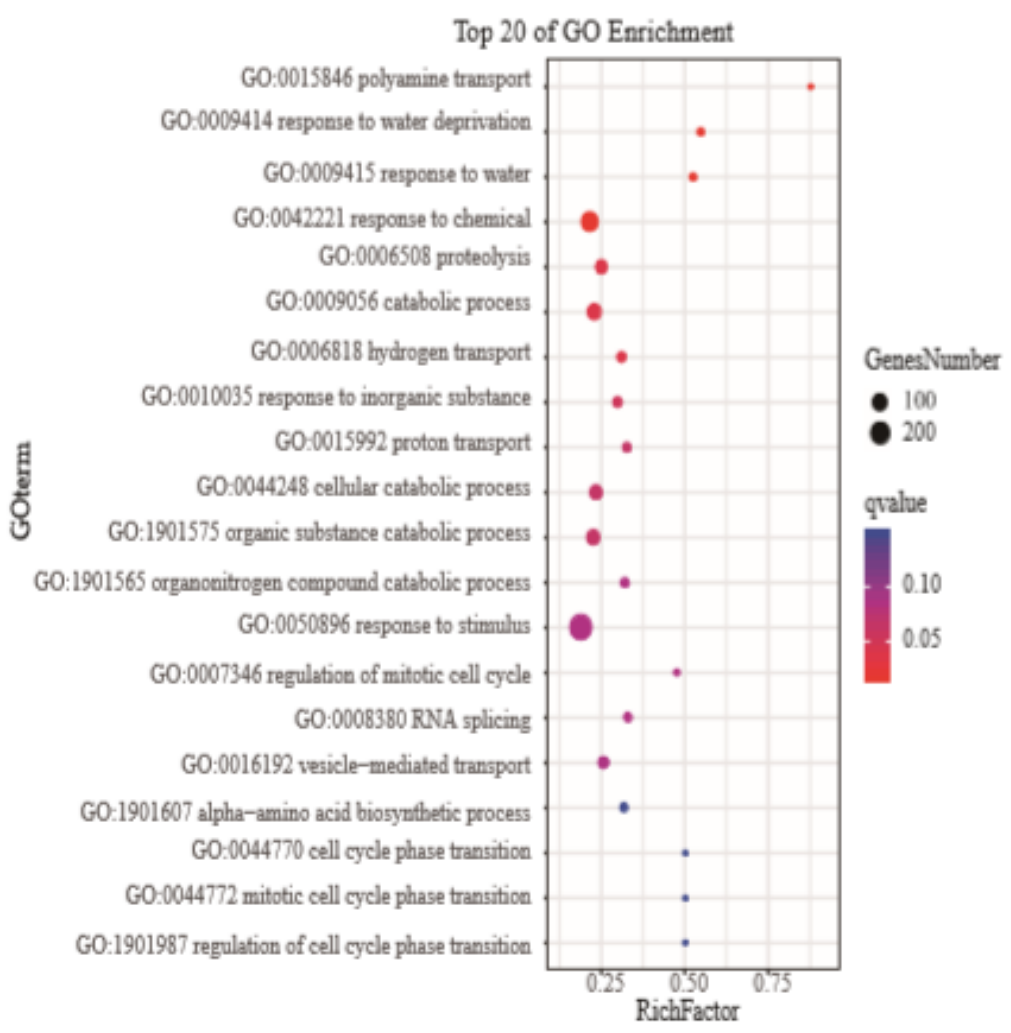

B

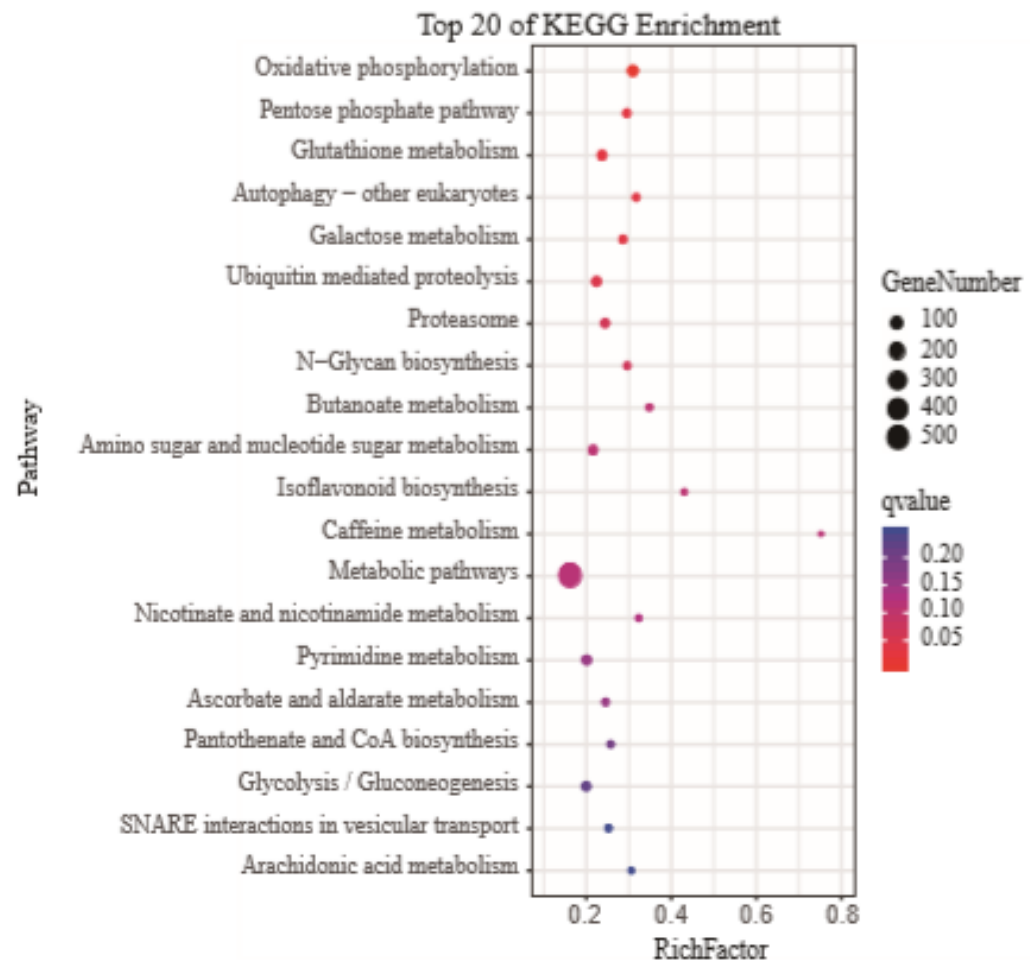

Figure 13

GO and KEGG analysis of differentially expressed genes in the navajowhite1 module. (A) The top 20 of GO enrichment. (B) The top 20 of KEGG enrichment. (The q value ranges from $0-0.05$. The closer the $q$ value is to 0 , the more significant the enrichment is. Genes number is the number of genes enriched in pathways.) 
A

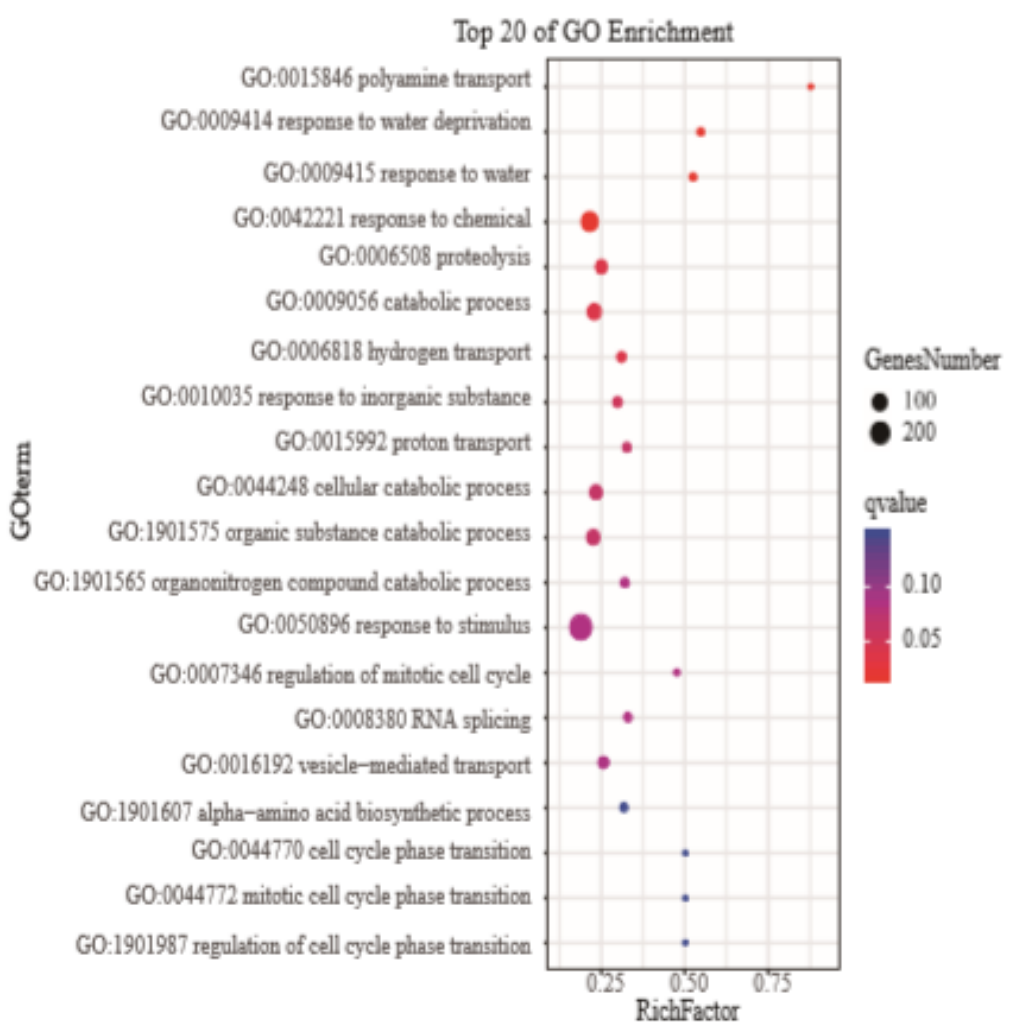

B

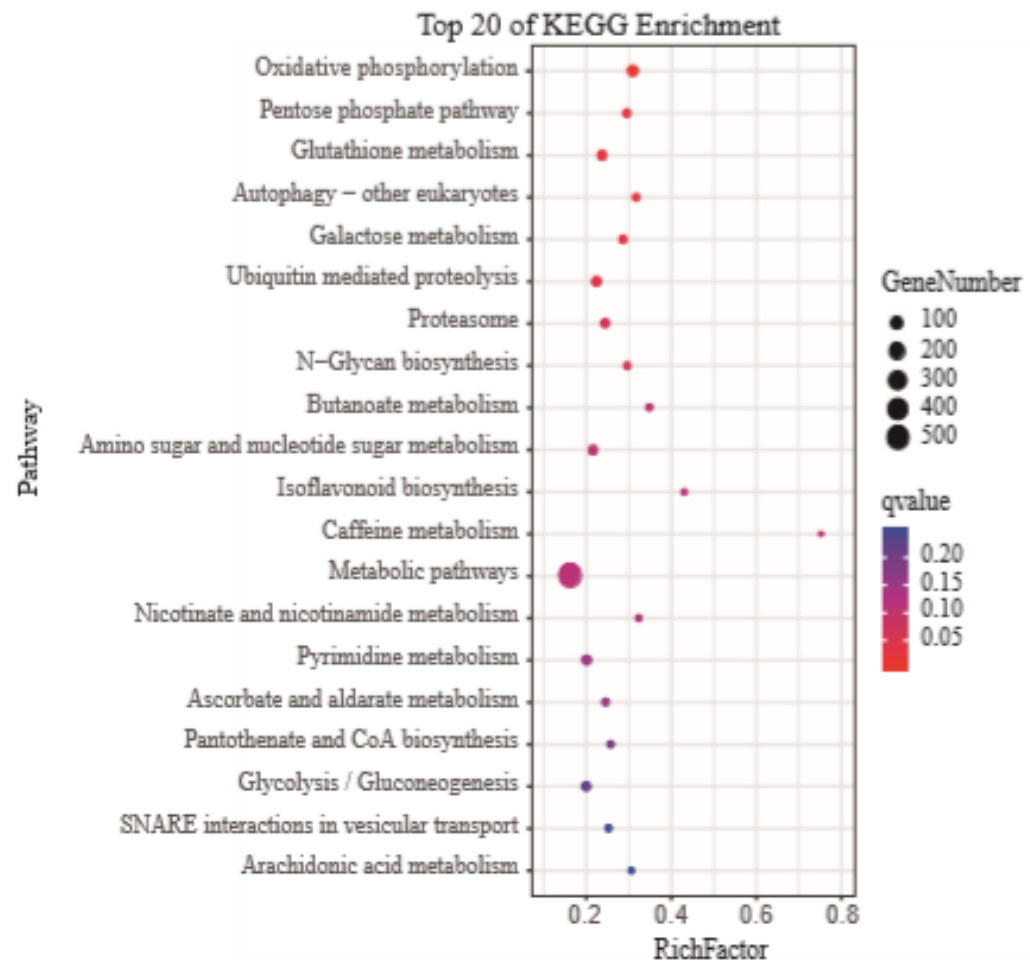

Figure 13

GO and KEGG analysis of differentially expressed genes in the navajowhite1 module. (A) The top 20 of GO enrichment. (B) The top 20 of KEGG enrichment. (The q value ranges from $0-0.05$. The closer the $q$ value is to 0 , the more significant the enrichment is. Genes number is the number of genes enriched in pathways.) 


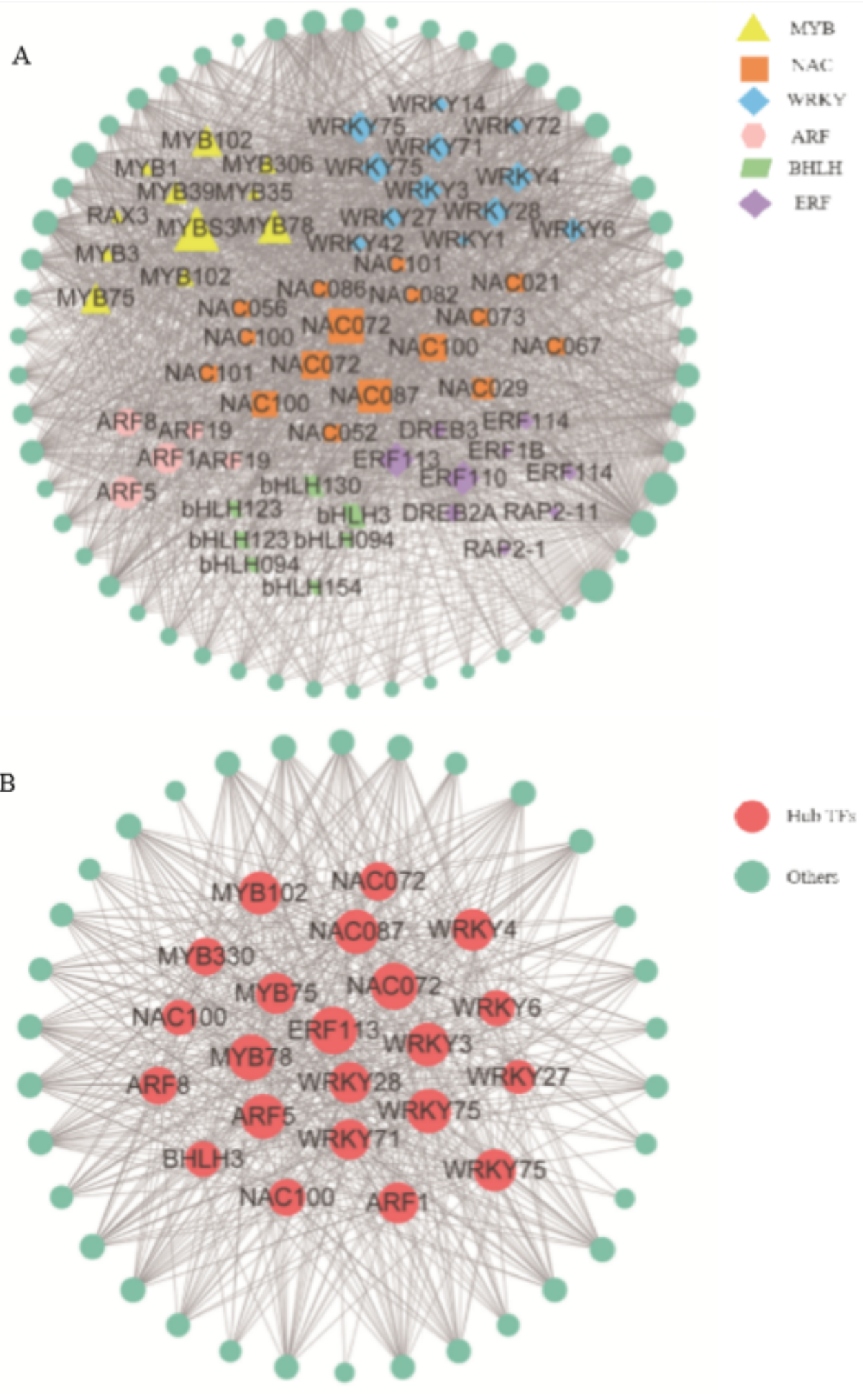

\section{Figure 14}

Identification and selection of vital transcription factors in the navajowhite1 module. (A) Network analysis of TFs in navajowhite1 module. (B) Network of the top 22 hub TFs and related genes in the navajowhite1 module. 


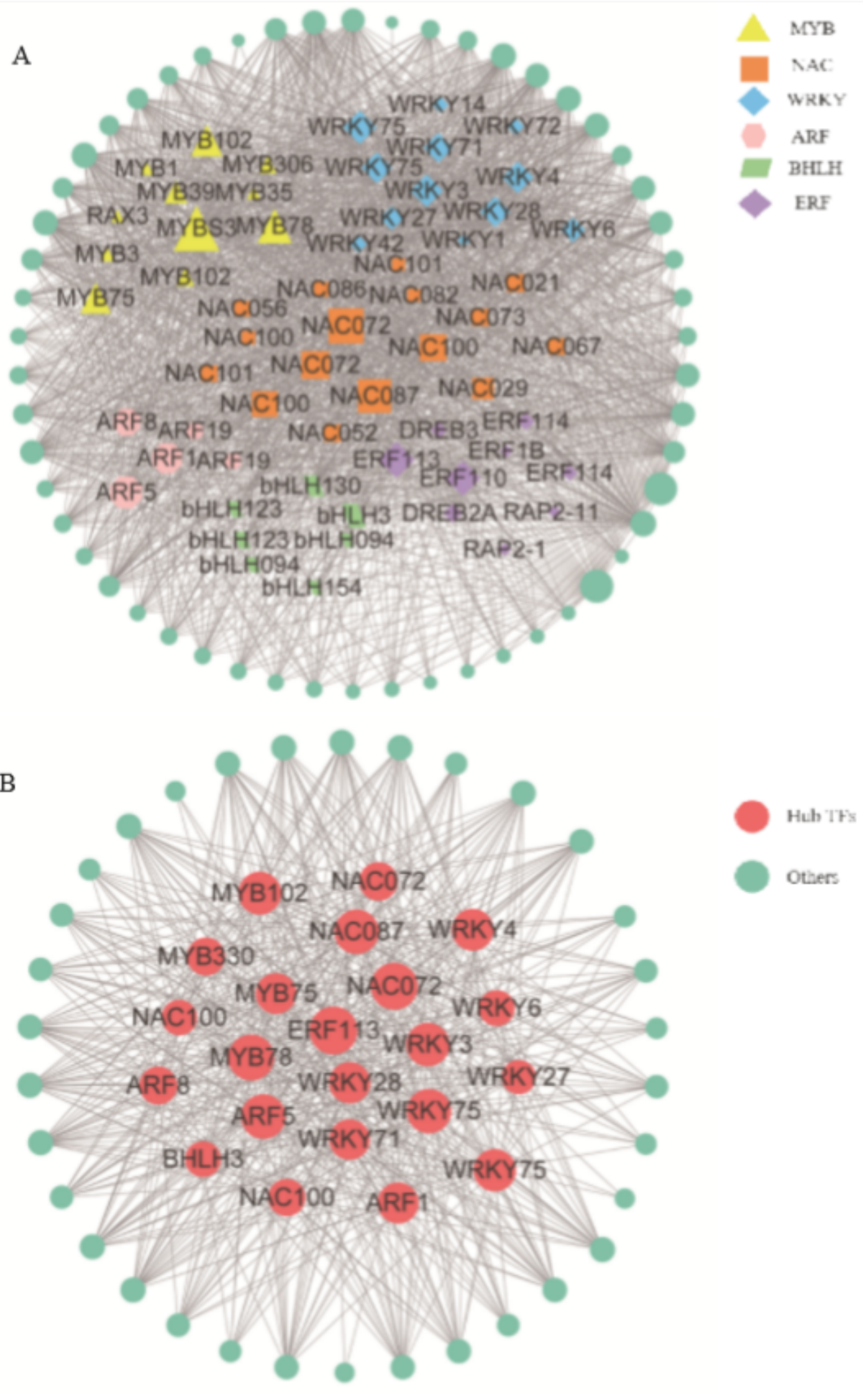

\section{Figure 14}

Identification and selection of vital transcription factors in the navajowhite1 module. (A) Network analysis of TFs in navajowhite1 module. (B) Network of the top 22 hub TFs and related genes in the navajowhite1 module. 


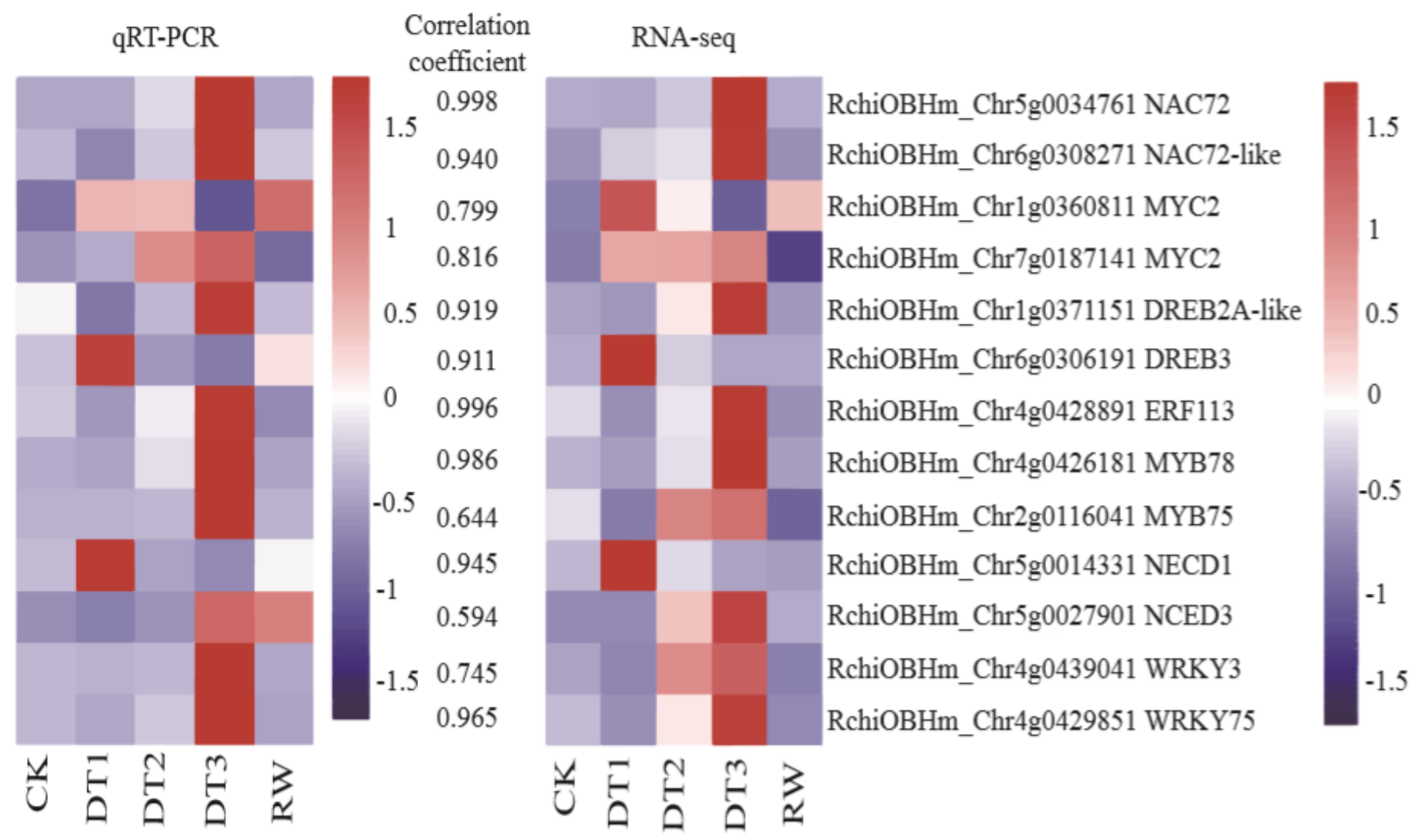

Figure 15

Heatmaps of the validation and correlation analysis of 13 selected DEGs. The values between two heatmaps represent the correlation coefficients of qRT-PCR and RNA-seq values from each gene. 


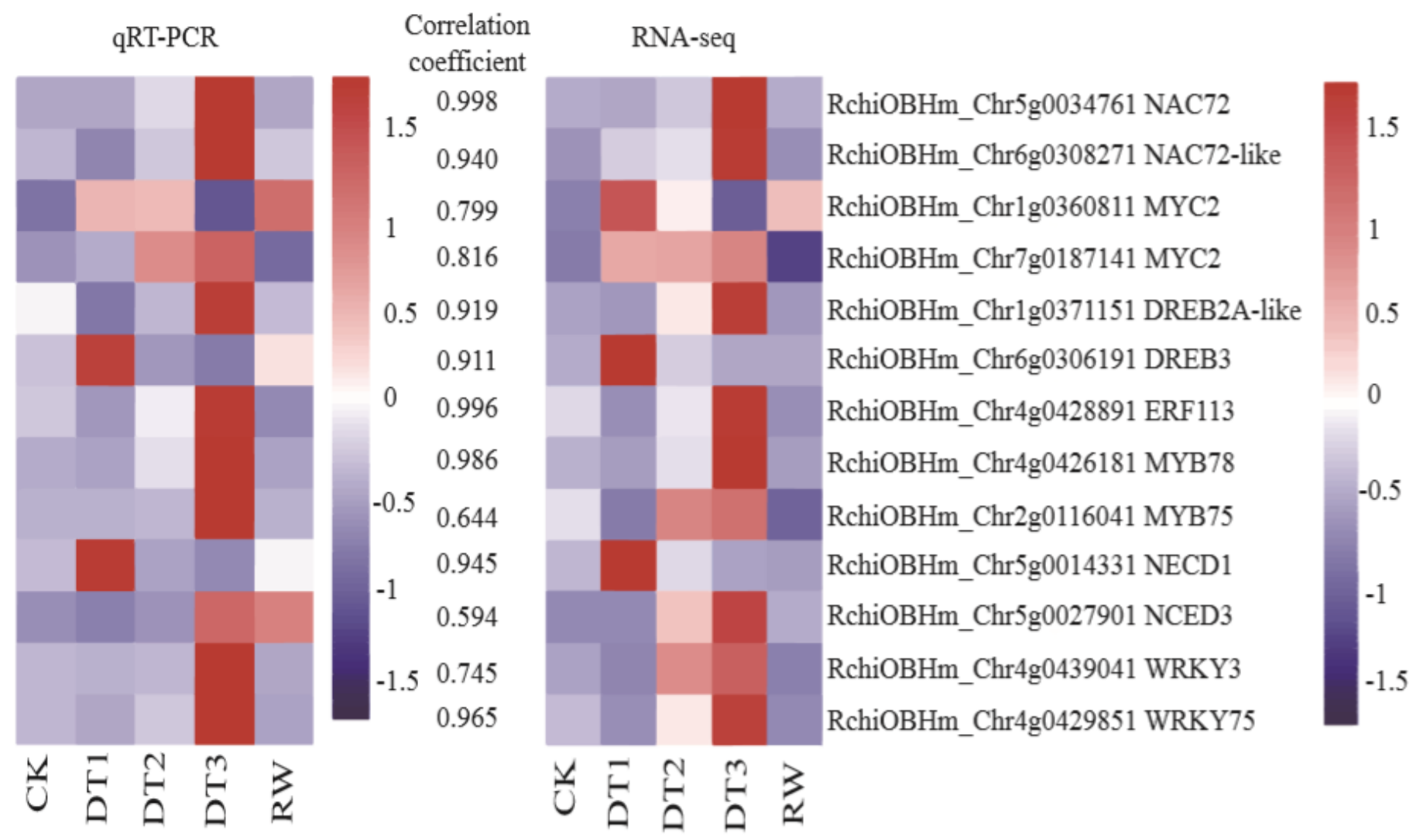

Figure 15

Heatmaps of the validation and correlation analysis of 13 selected DEGs. The values between two heatmaps represent the correlation coefficients of qRT-PCR and RNA-seq values from each gene. 


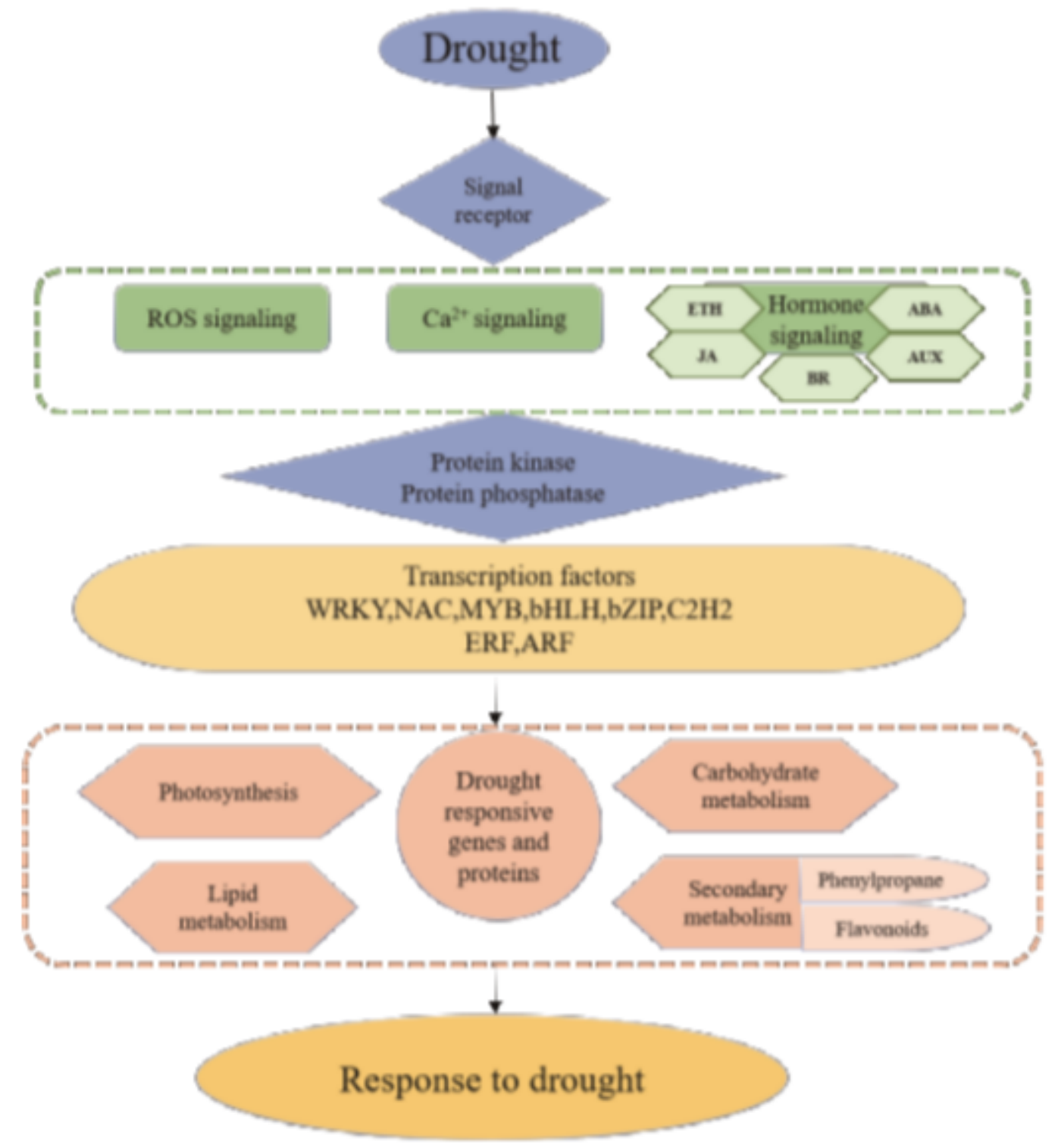

Figure 16

The hypothetical model of Rosa chinensis 'Old Blush' in response to drought stress. 


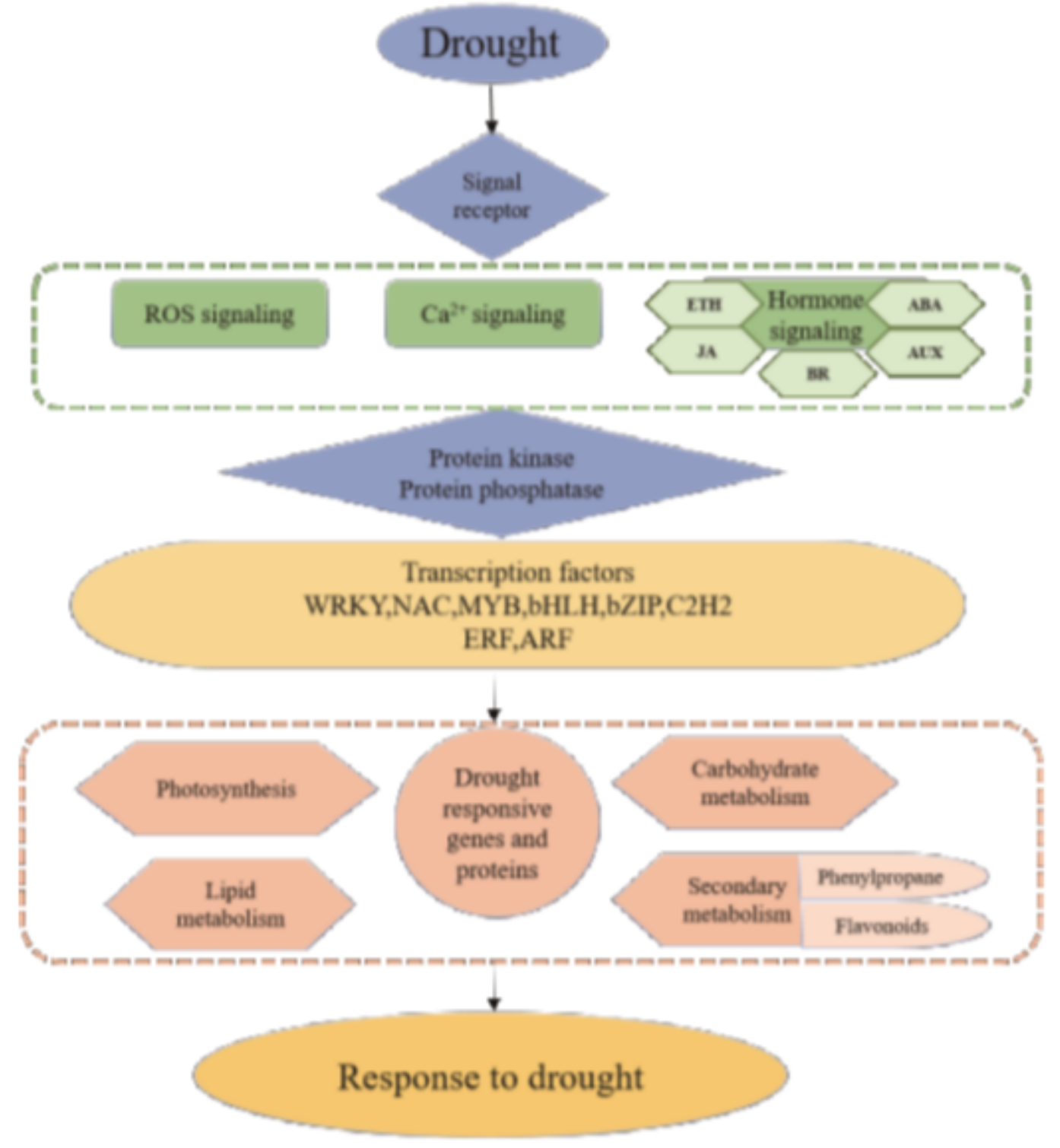

Figure 16

The hypothetical model of Rosa chinensis 'Old Blush' in response to drought stress.

\section{Supplementary Files}

This is a list of supplementary files associated with this preprint. Click to download.

- Additionalfile1FigureS1S4.docx

- Additionalfile1FigureS1S4.docx

- Additionalfile10TableS9.xlsx

- Additionalfile10TableS9.xlsx

- Additionalfile11TableS10.xlsx

- Additionalfile11TableS10.xIsx 
- Additionalfile12TableS11.xlsx

- Additionalfile12TableS11.xlsx

- Additionalfile13Tables12.xlsx

- Additionalfile13TableS12.xIsx

- Additionalfile2TableS1.xlsx

- Additionalfile2TableS1.xlsx

- Additionalfile3Tables2.xlsx

- Additionalfile3Tables2.xlsx

- Additionalfile4TableS3.xlsx

- Additionalfile4TableS3.xIsx

- Additionalfile5TableS4.xlsx

- Additionalfile5TableS4.xIsx

- Additionalfile6TableS5.xIsx

- Additionalfile6TableS5.xlsx

- Additionalfile7TableS6.xIsx

- Additionalfile7TableS6.xlsx

- Additionalfile8TableS7.xlsx

- Additionalfile8TableS7.xIsx

- Additionalfile9TableS8.xlsx

- Additionalfile9TableS8.xlsx 